\title{
Temperatureffekte bei der lasererzeugten Kavitation
}

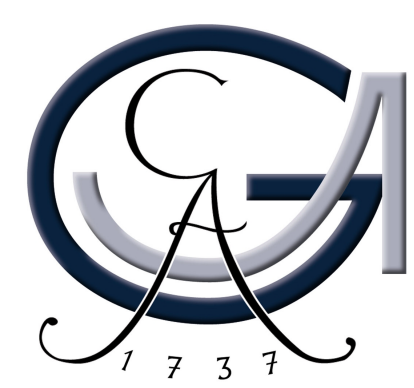

\section{Dissertation}

zur Erlangung des mathematisch-naturwissenschaftlichen Doktorgrades "Doctor rerum naturalium" der Georg-August-Universität Göttingen

im Promotionsprogramm PROPHYS der Georg-August University School of Science (GAUSS)

vorgelegt von

Hendrik Söhnholz

aus

Großburgwedel

Göttingen 2016 
Betreuungsausschuss:

Prof. Dr. Christoph Schmidt Prof. Dr. Andreas Tilgner Dr. Thomas Kurz
Drittes Physikalisches Institut Institut für Geophysik

Drittes Physikalisches Institut
Universität Göttingen Universität Göttingen Universität Göttingen

Mitglieder der Prüfungskommission:

Referent:

Prof. Dr. Christoph Schmidt Drittes Physikalisches Institut Universität Göttingen

Korreferent:

Prof. Dr. Ulrich Parlitz

MPI für Dynamik und Selbstorganisation

Göttingen

Weitere Mitglieder der Prüfungskommission:

Dr. Thomas Kurz

Prof. Dr. Andreas Tilgner

Drittes Physikalisches Institut

Institut für Geophysik

Prof. Dr. Wolfram Kollatschny

Prof. Dr. Stefan Luther

Institut für Astrophysik

MPI für Dynamik

und Selbstorganisation
Universität Göttingen Universität Göttingen Universität Göttingen Göttingen

Tag der mündlichen Prüfung: 26. Oktober 2016 


\section{Inhaltsverzeichnis}

Symbolverzeichnis v v

$\begin{array}{lll}1 & \text { Einleitung } & 1\end{array}$

2 Blasendynamik in Theorie und Experiment 3

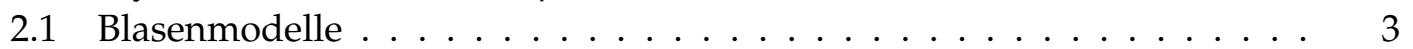

2.2 Blasendynamik in Wasser bei erhöhten Temperaturen . . . . . . . . . . . 12

2.3 Wärmetransport über die Blasenwand . . . . . . . . . . . . . . . . . . . 15

2.4 Lasererzeugte Kavitation . . . . . . . . . . . . . . . . . . . . . . 16

2.4.1 Blasenerzeugung im Experiment mit gepulsten Lasern . . . . . . 17

2.5 Akustische Blasenfallen und Sonochemie . . . . . . . . . . . . . . 18

3 Dynamik lasererzeugter Kavitationsblasen bei verschiedenen Wassertemperaturen 21

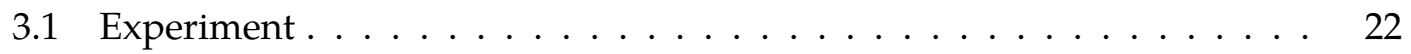

3.2 Numerische Simulationen . . . . . . . . . . . . . . . . . . . 26

3.2 .1 Gilmore Modell . . . . . . . . . . . . . . . . . . . . . . . . . 27

3.2 .2 Eick Modell . . . . . . . . . . . . . . . . . . . . 27

$3.2 .3 \quad$ Toegel Modell . . . . . . . . . . . . . . . . . . . . . . 28

3.2.4 Vergleich der verschiedenen Blasenmodelle. . . . . . . . . . . . . . . 28

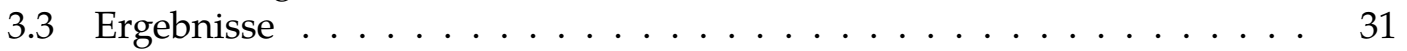

3.3.1 Hochgeschwindigkeitsaufnahmen . . . . . . . . . . . . . . 31

3.3.2 Ergebnisse der numerischen Rechnungen und Vergleich mit den

3.4 Ausblick . . . . . . . . . . . . . . . . . . . 41

4 Temperaturmessung mittels laserinduzierter Fluoreszenz 47

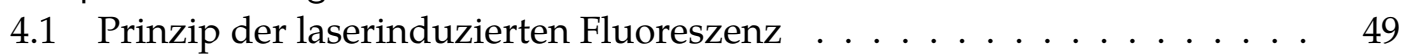

4.2 Experimenteller Aufbau $\ldots \ldots \ldots \ldots \ldots \ldots$. . . . . . . . . . . . . . . . . . . . . . . 52

4.3 Auswertung . . . . . . . . . . . . . . . . . . . . 53

4.4 Vorexperimente, Testen der LIF-Methode . . . . . . . . . . . . . . . . . 55

4.4 .1 Messung der Lichtschnittdicke . . . . . . . . . . . . . . . . 55

4.4.2 Überprüfung der Bandpassfilter, Übersprechen . . . . . . . . . . 56

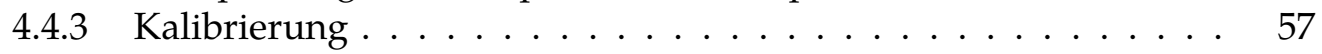


$4.4 .4 \quad$ Lokales Heizen mit kleiner Spule . . . . . . . . . . . . . . . . . . . 59

4.4 .5 Glasküvette mit Peltierelement an einer Seite . . . . . . . . . . . . 60

4.4.6 Abhängigkeit der Fluoreszenzintensität von der Energie des An-

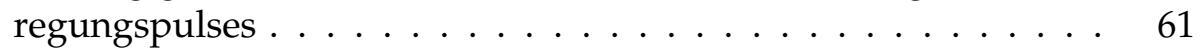

4.4 .7 Ausbleichen der Farbstoffe (Photobleaching) . . . . . . . . . . . . 63

4.5 LIF-Messung an laserinduzierter Blase . . . . . . . . . . . . . . . . . . . . . . 64

$4.6 \quad$ LIF-Messung an einer Blase im Schallfeld . . . . . . . . . . . . . . . . . . 67

4.7 Ausblick $\ldots \ldots \ldots \ldots \ldots$

$\begin{array}{lll}5 & \text { Zusammenfassung und Ausblick } & 71\end{array}$

5.1 Zusammenfassung . . . . . . . . . . . . . . . . . . . . 71

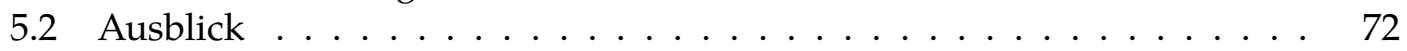

A Stoßwellen beim Blasenkollaps vor einer festen Wand 75

A.1 Stoßwellenaufnahmen im Schlierenverfahren . . . . . . . . . . . . . . . . 75

A.2 Stoßwellenaufnahmen im Schattenverfahren . . . . . . . . . . . . . . 76

A.3 Ausblick . . . . . . . . . . . . . . . . . . . . 78

B Linsensysteme zur Blasenerzeugung mit gepulsten Lasern 81

C Konstanten, Materialeigenschaften und Parameter 83

\begin{tabular}{ll}
\hline Literaturverzeichnis & 85
\end{tabular} 


\section{Symbolverzeichnis}

In der folgenden Tabelle sind die im Text verwendeten Symbole aufgelistet. Konstanten, Materialeigenschaften und Parameter sind in Anhang Caufgeführt. 


\begin{tabular}{|c|c|c|}
\hline Symbol & Einheit & Bezeichnung \\
\hline$\alpha$ & $\mathrm{m}^{2} \mathrm{~s}^{-1}$ & Temperaturleitfähigkeit \\
\hline$\beta$ & - & Van-der-Waals Koeffizient \\
\hline$\gamma$ & - & normierter Abstand Blase-Wand \\
\hline$\kappa$ & - & Polytropenexponent \\
\hline$\lambda$ & $\mathrm{m}$ & Wellenlänge \\
\hline$\mu$ & Pas & (dynamische) Viskosität der Flüssigkeit \\
\hline$\varrho$ & $\mathrm{kg} \mathrm{m}^{-3}$ & Dichte \\
\hline$\sigma$ & $\mathrm{Nm}^{-1}$ & Oberflächenspannung \\
\hline$\chi$ & $\mathrm{Pa}^{-1}$ & Kompressibilität \\
\hline$A$ & $\mathrm{~Pa}$ & Tait Parameter \\
\hline$a$ & $\mathrm{~m}$ & Van-der-Waals Parameter \\
\hline$B$ & $\mathrm{~Pa}$ & Tait Parameter \\
\hline$b$ & $\mathrm{~m}^{3} \mathrm{~mol}^{-1}$ & Van-der-Waals Kovolumen \\
\hline$C$ & $\mathrm{moll}^{-1}$ & Konzentration \\
\hline$C$ & $\mathrm{~ms}^{-1}$ & Schallgeschwindigkeit an der Blasenwand \\
\hline$c$ & $\mathrm{~m} \mathrm{~s}^{-1}$ & Schallgeschwindigkeit \\
\hline$c_{0}$ & $\mathrm{~m} \mathrm{~s}^{-1}$ & Schallgeschwindigkeit unter Normalbedingungen \\
\hline$c_{p}$ & $\mathrm{~J} \mathrm{~kg}^{-1} \mathrm{~K}^{-1}$ & spezifische Wärmekapazität (bei konstantem Druck) \\
\hline$D$ & $\mathrm{~m}^{2} \mathrm{~s}^{-1}$ & Diffusionskoeffizient \\
\hline$H$ & $\mathrm{~J}$ & Enthalpie \\
\hline$k$ & $\mathrm{~W} \mathrm{~m}{ }^{-1} \mathrm{~K}^{-1}$ & Wärmeleitfähigkeit \\
\hline$k_{\mathrm{g}}$ & $\mathrm{W} \mathrm{m}^{-1} \mathrm{~K}^{-1}$ & Wärmeleitfähigkeit des Gases \\
\hline$N$ & mol & Stoffmenge \\
\hline$n$ & $\mathrm{~mol} \mathrm{~m}^{-3}$ & Stoffmengendichte \\
\hline$n_{\mathrm{T}}$ & - & Tait Parameter (Exponent) \\
\hline$p_{\text {ext }}$ & $\mathrm{Pa}$ & extern angelegter Druck (z. B. Schallanregung) \\
\hline$p_{\mathrm{g}}$ & $\mathrm{Pa}$ & Gasdruck in der Blase \\
\hline$p_{\text {stat }}$ & $\mathrm{Pa}$ & statischer Druck \\
\hline$p_{\mathrm{V}}$ & $\mathrm{Pa}$ & Dampfdruck \\
\hline$R$ & $\mathrm{~m}$ & Blasenradius \\
\hline$R_{\max }$ & $\mathrm{m}$ & maximaler Blasenradius (global) \\
\hline$R_{\max , \mathrm{Rb} 1}$ & $\mathrm{~m}$ & maximaler Blasenradius im 1. Rebound \\
\hline$R_{\mathrm{n}}$ & $\mathrm{m}$ & Gleichgewichts- oder Ruheradius der Blase \\
\hline$R_{\mathrm{g}}$ & $\mathrm{J} \mathrm{mol}^{-1} \mathrm{~K}^{-1}$ & universelle Gaskonstante \\
\hline$T^{\circ}$ & $\mathrm{K}$ & Temperatur \\
\hline$T_{l}$ & $\mathrm{~K}$ & Temperatur in der Flüssigkeit \\
\hline $\mathrm{Nu}$ & - & Nusseltzahl \\
\hline $\operatorname{Pr}$ & - & Prandtlzahl \\
\hline $\operatorname{Re}$ & - & Reynoldszahl \\
\hline
\end{tabular}




\section{Einleitung}

Eine Blase ist ein mit Gas und Dampf gefüllter Hohlraum in einer Flüssigkeit. Die Entstehung von Blasen durch Aufreißen einer Flüssigkeit (aufgrund von hohen Zugspannungen oder Energieeintrag) wird als Kavitation bezeichnet.

Bereits vor etwa 100 Jahren wurde Erosion an Schiffsschrauben beobachtet und es stellte sich heraus, dass kollabierende Kavitationsblasen dafür verantwortlich waren [1]. Kavitationserosion kann außerdem in Pumpen, Turbinen, Rohrleitungen sowie Einspritzdüsen von Motoren auftreten. Bis heute ist der Erosionsmechanismus noch nicht genau verstanden. Jedoch spielen die Jetbildung und die Stoßwellenabstrahlung beim asphärischen Blasenkollaps vor einer festen Oberfläche dabei eine wichtige Rolle [2, 3].

Blasen können, beispielsweise in einem Ultraschallbad, zur Reinigung von verschmutzten Oberflächen beitragen. Solche Ultraschallbäder werden oft zur Reinigung von Brillen und Schmuck verwendet. In der Halbleiterindustrie wird zur Reinigung von Siliziumwafern im Laufe der Chipherstellung auch Ultraschall eingesetzt.

Für verschiedene medizinische Anwendungen sind Blasen wichtig. In der Augenheilkunde (Ophthalmologie) können bei Laseroperationen Kavitationsblasen im Auge entstehen [4]. Beim bildgebenden Ultraschall sorgen Mikrobläschen als Kontrastmittel für einen erhöhten Kontrast und damit für eine verbesserte Bildqualität. Mikrobläschen können auch als Behälter für Medikamente dienen, die anschließend durch die Anregung mit Ultraschall freigesetzt werden [5]. Bei der Sonoporation werden Zellmembranen durch im Ultraschallfeld schwingende Blasen geöffnet, so dass Medikamente von den Zellen aufgenommen werden können [6].

An den oben genannten Anwendungen sind üblicherweise sehr viele Blasen beteiligt. Zur theoretischen und experimentellen Untersuchung der Blasendynamik bietet es sich an, zunächst eine einzelne Blase zu betrachten. Eine solche lässt sich u. a. mit einem gepulsten Laser kontrolliert und reproduzierbar erzeugen [7]. Diese Arbeit beschäftigt sich vor allem mit einzelnen lasererzeugten Blasen in Wasser. 
Ein Schwerpunkt liegt auf der Untersuchung der Einzelblasendynamik in Abhängigkeit von der Wassertemperatur. Dazu gibt es bereits theoretische und experimentelle Untersuchungen. Allerdings wurden dabei meist niedrige Wassertemperaturen betrachtet. In der vorliegenden Arbeit wird daher die Dynamik von einzelnen lasererzeugten Blasen bei Wassertemperaturen von bis $\mathrm{zu} 80^{\circ} \mathrm{C}$ mit einer Hochgeschwindigkeitskamera aufgenommen. Außerdem werden zum Vergleich numerische Rechnungen mit verschiedenen Modellen für kugelförmige Blasen durchgeführt.

Aufgrund des starken Anstiegs des Dampfdrucks von Wasser erwartet man bei hohen Temperaturen einen erhöhten Dampfgehalt im Blaseninnern (im Vergleich zur Menge nicht kondensierbaren Gases wie z. B. Sauerstoff). Fujikawa und Akamatsu [8] haben bei ihren numerischen Rechnungen festgestellt, dass der Dampf und das Gas in der Blase einen großen Einfluss auf ihre Dynamik haben.

Wegen der extrem hohen Temperaturen innerhalb der Blase beim Kollaps kann man davon ausgehen, dass Wärmetransport von der Blase in die Flüssigkeit stattfindet. Dies soll im Experiment durch Temperaturmessungen in der Flüssigkeit nahe der Blase untersucht werden. Dazu kommt die Methode der laserinduzierten Fluoreszenz zum Einsatz, bei der ein im Wasser gelöster Fluoreszenzfarbstoff zur Temperaturmessung dient.

Es gibt noch weitere interessante Blasenphänomene, die aber nicht Teil dieser Arbeit sind. Eine schwingende Blase ist im Prinzip ein nichtlinearer Oszillator und kann auch chaotisches Verhalten aufweisen [9]. In Mehrblasensystemen, beispielsweise in einem Ultraschallbad, können sich durch die Wechselwirkung der Blasen bestimmte Strukturen ausbilden [10].

An diese Einleitung schließt sich das Kap. 2 an, in dem einige theoretische und experimentelle Grundlagen zur Dynamik von einzelnen Blasen in Wasser besprochen werden. Kapitel 3 beschreibt ein Experiment zur Untersuchung von lasererzeugten Blasen in Wasser bei erhöhten Temperaturen mit einer Hochgeschwindigkeitskamera. Die Ergebnisse des Experiments werden mit numerischen Rechnungen verglichen. Ein Experiment zur Temperaturmessung an einzelnen Blasen mittels laserinduzierter Fluoreszenz wird in Kap. 4 vorgestellt. Es werden Ergebnisse von Messungen an lasererzeugten Blasen sowie an im Schallfeld eingefangenen Blasen gezeigt. Schließlich fasst Kap. 5 die gesamte Arbeit zusammen und gibt einen Ausblick auf zukünftige Untersuchungen. Zusätzlich werden in Anhang A zwei Experimente zur Visualisierung von Stoßwellen beschrieben und es werden einige damit gewonnene Aufnahmen gezeigt. 


\section{Blasendynamik in Theorie und Experiment}

Dieses Kapitel behandelt einige Grundlagen zur Dynamik von einzelnen Blasen in Flüssigkeiten. Es werden verschiedene Modelle zur Beschreibung der Dynamik von sphärischen Blasen vorgestellt. Dabei wird insbesondere auf den Einfluss der Wassertemperatur eingegangen. Außerdem wird der Wärmetransport über die Grenzfläche BlaseFlüssigkeit während der Blasenschwingung betrachtet.

Schließlich werden noch zwei experimentelle Methoden zur Untersuchung von einzelnen Blasen vorgestellt: lasererzeugte Kavitation und akustische Blasenfallen. Letztere spielen eine wichtige Rolle bei der Einzelblasensonolumineszenz (SBSL) sowie in der Sonochemie.

\subsection{Blasenmodelle}

Dieser Abschnitt basiert zu einem großen Teil auf einem Übersichtsartikel von Lauterborn und Kurz [10]. Eine schwingende Blase wird durch eine Druckdifferenz zwischen dem Blaseninneren (Druck $p_{\mathrm{i}}$ ) und der Umgebung (Druck $p_{\mathrm{e}}$ ) angetrieben. Typischerweise wächst der Blasenradius (Abb. 2.1, oben) zunächst bis zu einem gewissen Maximalradius $R_{\max }$ an. Anschließend kollabiert die Blase, der Blasenradius wird also kleiner. Dabei kann die Geschwindigkeit der Blasenwand $\dot{R}$ sehr hohe Werte annehmen und der Blasenradius $R$ sehr klein werden. Im Kollaps, d. h. wenn der Radius minimal wird, können aufgrund der starken Kompression Stoßwellen entstehen, die sich kugelförmig ausbreiten. Das untere Diagramm in Abb. 2.1 zeigt ein Hydrofonsignal, in dem die Stoßwellen als Spitzenwerte zu erkennen sind. Auch im Laserdurchbruch, also bei der Entstehung der Blase, werden Stoßwellen abgestrahlt. Die Stoßwellen treten im Hydrofonsignal mit einer Verzögerung von wenigen Mikrosekunden auf, da sie erst die Strecke von der Blase bis zum Hydrofon zurücklegen müssen.

Der zeitliche Abstand vom Auftreten des Maximalradius $R_{\max }$ bis zum Kollaps wird als Kollapszeit $t_{c}$ bezeichnet (siehe Abb. 2.1). Zwischen der Blasenerzeugung und dem 

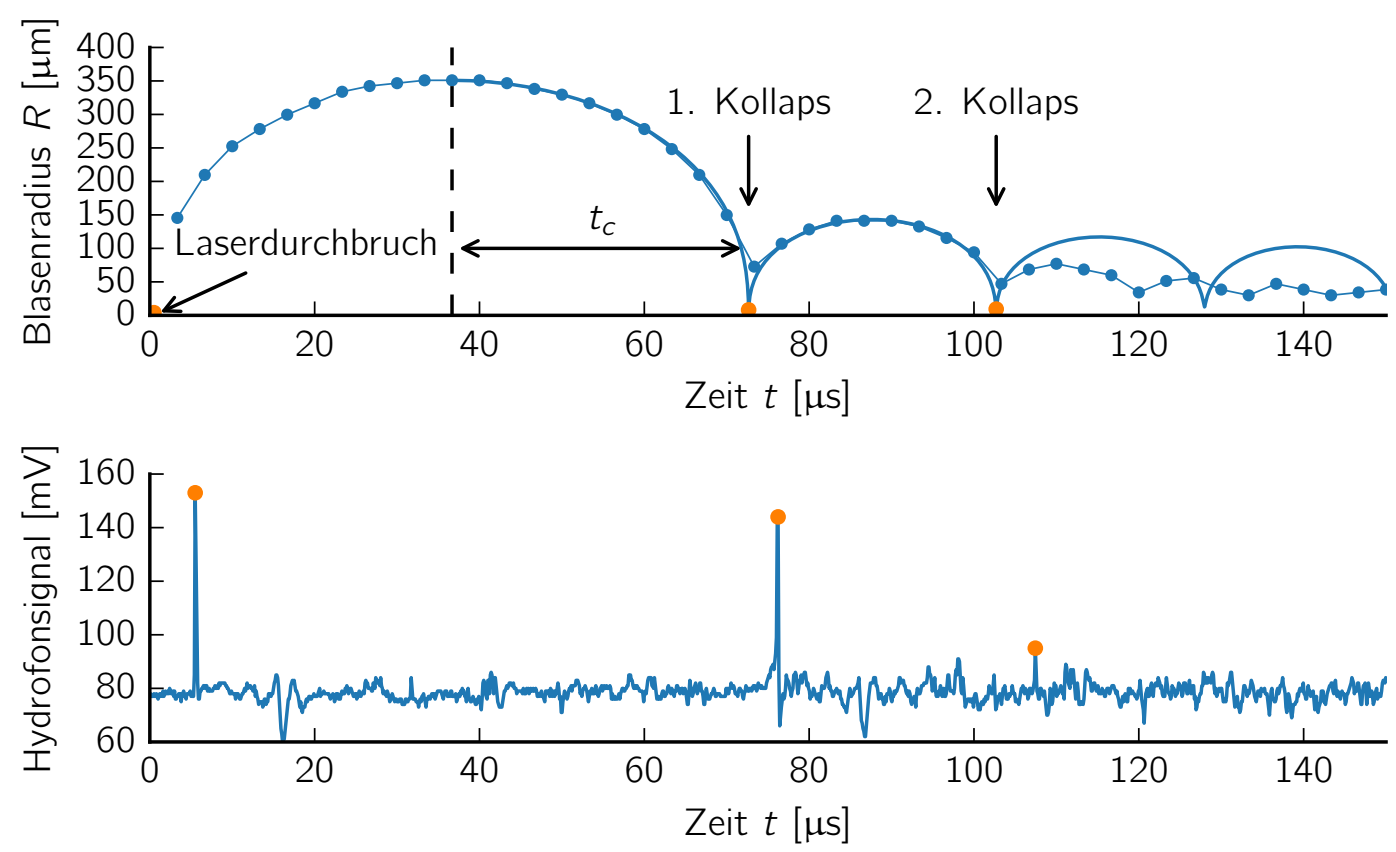

Abbildung 2.1: Typische Schwingung einer einzelnen lasererzeugten Blase in Wasser (oben) und akustische Emissionen der Blase (unten). Im oberen Diagramm stellen die Punkte gemessene Blasenradien dar; die durchgezogene Linie wurde mit Hilfe des Gilmore Modells numerisch berechnet.

1. Kollaps liegt bei symmetrischem Aufschwingen und Kollabieren die doppelte Kollapszeit $2 t_{c}$.

Zur theoretischen Beschreibung der Dynamik von sphärischen Blasen gibt es mittlerweile eine Vielzahl von Modellen. Die meisten davon gehen auf eine Arbeit von Lord Rayleigh [11] zurück, in der er den Kollaps einer leeren Blase in einer inkompressiblen Flüssigkeit unter dem hydrostatischen Druck $p_{\text {stat }}$ beschreibt. Im Rayleigh Modell gilt für den Blasenradius [10]:

$$
\varrho R \ddot{R}+\frac{3}{2} \varrho \dot{R}^{2}=p_{\mathrm{i}}-p_{\mathrm{e}}
$$

Bei gegebenem Maximalradius, gleichbleibender Dichte $\varrho$ und gegebenem statischem Druck $p_{\text {stat }}$ ergibt sich daraus die Kollapszeit [12]

$$
t_{c}=0.915 R_{\max } \sqrt{\frac{\varrho}{p_{\text {stat }}}} \propto R_{\max }
$$


Darauf aufbauend wurden später die Modelle von Rayleigh-Plesset, Keller-Miksis und Gilmore entwickelt [10,12]. Diese einfachen Modelle, die auf gewöhnlichen Differentialgleichungen basieren, berücksichtigen bereits die Oberflächenspannung, die Viskosität der Flüssigkeit und ihren Dampfdruck sowie, bei Keller-Miksis und Gilmore, auch die Schallabstrahlung von der Blase unter der Annahme, dass die Flüssigkeit schwach kompressibel ist. Sie beschreiben die Dynamik von sphärischen Blasen in den meisten Fällen sehr gut.

Das Rayleigh-Plesset Modell ergibt sich unter Berücksichtigung des Dampfdrucks, der Oberflächenspannung und der Viskosität der Flüssigkeit aus dem Rayleigh Modell:

$$
\varrho R \ddot{R}+\frac{3}{2} \varrho \dot{R}^{2}=p_{\text {gn }}\left(\frac{R_{\mathrm{n}}}{R}\right)^{3 \kappa}+p_{\mathrm{v}}-\frac{2 \sigma}{R}-\frac{4 \mu \dot{R}}{R}-p_{\text {stat }}-p_{\text {ext }}(t)
$$

Dabei ist

$$
p_{\text {gn }}=\frac{2 \sigma}{R_{\mathrm{n}}}+p_{\text {stat }}-p_{\mathrm{v}}
$$

der Gasdruck in der Blase im Gleichgewicht. Die Anregung kann z. B. von folgender Form sein:

$$
p_{\text {ext }}(t)=-p_{\mathrm{a}} \sin \left(2 \pi f_{\mathrm{a}} t\right)
$$

Für frei schwingende Blasen ist $p_{\text {ext }}(t)=0$.

Im Gilmore Modell [13] wird zusätzlich die Flüssigkeit als schwach kompressibel betrachtet; es beinhaltet damit die Schallabstrahlung von der Blase. Durch die Einführung eines Van-der-Waals Gesetzes für das Blaseninnere wird ein nicht komprimierbarer Gasanteil berücksichtigt. Die Gilmore Gleichung lautet

$$
\left(1-\frac{\dot{R}}{C}\right) R \ddot{R}+\frac{3}{2}\left(1-\frac{\dot{R}}{3 C}\right) \dot{R}^{2}=\left(1+\frac{\dot{R}}{C}\right) H+\frac{\dot{R}}{C}\left(1-\frac{\dot{R}}{C}\right) R \frac{\mathrm{d} H}{\mathrm{~d} R}
$$

mit

$$
\begin{aligned}
H & =\int_{\left.p\right|_{r \rightarrow \infty}}^{\left.p\right|_{r=R}} \frac{\mathrm{d} p(\varrho)}{\varrho} \\
p(\varrho) & =A\left(\frac{\varrho}{\varrho_{0}}\right)^{n_{\mathrm{T}}}-B \\
\left.p\right|_{r=R} & =\left(p_{\text {stat }}-p_{\mathrm{v}}+\frac{2 \sigma}{R_{\mathrm{n}}}\right)\left(\frac{R_{\mathrm{n}}^{3}-\beta R_{\mathrm{n}}^{3}}{R^{3}-\beta R_{\mathrm{n}}^{3}}\right)^{\kappa}+p_{\mathrm{v}}-\frac{2 \sigma}{R}-\frac{4 \mu}{R} \dot{R} \\
\left.p\right|_{r \rightarrow \infty} & =p_{\text {stat }}+p_{\text {ext }}(t) \\
C & =\sqrt{c_{0}^{2}+\left(n_{\mathrm{T}}-1\right) H} .
\end{aligned}
$$


Als Zustandsgleichung für die Flüssigkeit wird die Tait Gleichung (2.7) verwendet. Das Gas in der Blase wird durch den Van-der-Waals Term am Anfang von Gl. (2.8) beschrieben. In diesem Modell treten zusätzlich die Enthalpie $H$, die Schallgeschwindigkeit unter Normalbedingungen $c_{0}$, die Schallgeschwindigkeit an der Blasenwand $C$, die Parameter der Tait Gleichung $A=\left.p\right|_{r \rightarrow \infty}+B, B$ und $n_{\mathrm{T}}$ sowie der Van-der-Waals Koeffizient $\beta$ auf.

Das Keller-Miksis Modell [14] ist vergleichbar mit dem Gilmore Modell und beinhaltet ebenfalls die Schallabstrahlung von der schwingenden Blase. Beide Modelle lassen sich mit Hilfe der Störungstheorie herleiten [15, 16]. Bei der Entwicklung nach der Machzahl $\mathrm{Ma}=\frac{\dot{R}}{C}$ kann man entscheiden, welche Terme mitgenommen und welche vernachlässigt werden. Die Keller-Miksis Gleichung enthält nur Terme 1. Ordnung in Ma, die Gilmore Gleichung enthält auch Terme 2. Ordnung. Allerdings sind in beiden Modellen die Fehler in $\mathcal{O}\left(\mathrm{Ma}^{2}\right)$.

Für eine genauere Beschreibung der Blasendynamik ist zunächst die Stoßwellenabstrahlung und die damit einhergehende Dämpfung der Blasenschwingung wichtig. Dazu muss im Modell die Kompressibilität der Flüssigkeit berücksichtigt werden. Bei Gilmore und Keller-Miksis wird bereits die Flüssigkeit als schwach kompressibel betrachtet; noch besser wäre es, die Flüssigkeit als voll kompressibel zu behandeln [15, 16, 17, 18]. Außerdem sollten Phasenübergänge (Verdampfung, Kondensation) sowie Stofftransport (Diffusion) im Modell enthalten sein, so dass sich Gas- und Dampfgehalt der Blase im Laufe der Simulation ändern können. Die Dämpfung der Blasenschwingung durch Wärmeleitung über die Blasenwand ist meist vernachlässigbar. Devin [19] vergleicht verschiedene Dämpfungsmechanismen bei einer Blasenschwingung in Wasser: Dämpfung durch Wärmeleitung, Schallabstrahlung (Stoßwellen) und viskose Reibung.

Das von Eick [20] verwendete Modell (im Folgenden kurz Eick Modell genannt) basiert auf dem Gilmore Modell und berücksichtigt zusätzlich die Wärmeleitung über die Blasenwand. Dazu wird ergänzend zu Gl. 2.6) eine Differentialgleichung für den Gasdruck $p_{\mathrm{g}}$ in der Blase eingeführt:

$$
\dot{p}_{\mathrm{g}}(\dot{R}, R, t)=\frac{-3 \kappa R^{2} \dot{R}}{R^{3}-\beta R_{\mathrm{n}}^{3}} p_{\mathrm{g}}(\dot{R}, R, t)+\frac{3}{2}(\kappa-1) k_{\mathrm{g}} \mathrm{Nu} \frac{T_{\mathrm{g} 0}-T_{\mathrm{g}}}{R^{2}}
$$

Dieser Ansatz stammt aus [21, 22] und wird auch in [23] verwendet. Dabei ist

$$
T_{\mathrm{g}}(R)=T_{\mathrm{g}}(0) \frac{p_{\mathrm{g}}(\dot{R}, R, t)}{p_{\mathrm{g}}(0)}\left(\frac{R}{R_{\mathrm{n}}}\right)^{3}
$$


die Temperatur innen an der Blasenwand und

$$
p_{\mathrm{g}}(0)=\underbrace{\left(p_{\mathrm{stat}}+\frac{2 \sigma}{R_{\mathrm{n}}}-p_{\mathrm{v}}\right)}_{p_{\mathrm{eq}}}\left(\frac{R_{\mathrm{n}}^{3}-\beta R_{\mathrm{n}}^{3}}{R(0)^{3}-\beta R_{\mathrm{n}}^{3}}\right)^{\kappa}
$$

der Anfangswert für den Gasdruck in der Blase. Außerdem treten hier die Nusseltzahl

$$
\mathrm{Nu}=0.111 \sqrt{\operatorname{Re}} \sqrt[3]{\operatorname{Pr}}
$$

die Reynoldszahl

$$
\operatorname{Re}=\frac{R_{\mathrm{n}}}{\mu_{\mathrm{g}}} \sqrt{\frac{p_{\infty}-p_{\mathrm{v}}}{\varrho_{0}}}
$$

sowie die Prandtlzahl

$$
\operatorname{Pr}=\frac{\mu_{\mathrm{g}} c_{p}}{k_{\mathrm{g}}}
$$

auf. Gleichung (2.8) wird ersetzt durch

$$
\left.p\right|_{r=R}=p_{\mathrm{g}}(\dot{R}, R, t)+p_{\mathrm{v}}-\frac{2 \sigma}{R}-\frac{4 \mu}{R} \dot{R}
$$

mit der neu eingeführten Variablen $p_{\mathrm{g}}(\dot{R}, R, t)$. Die Temperatur $T_{\mathrm{g}}$ in der Blase hängt wie durch Gl. 2.10 beschrieben vom Druck $p_{\mathrm{g}}$ und vom Blasenvolumen $\frac{4 \pi}{3} R^{3}$ ab. Die Temperatur $T_{1}$ der Flüssigkeit wird als konstant angenommen, da die Wärmeleitfähigkeit von Wasser viel höher ist als die des Gases in der Blase $\left(k_{1} \gg k_{\mathrm{g}}\right)$ und das Wasservolumen gegenüber dem Blasenvolumen viel größer ist. An der Blasenwand gilt die Randbedingung

$$
T_{\mathrm{g}}=T_{\mathrm{g}}(0)=T_{1}
$$

Temperatur $T_{\mathrm{g}}$ und Druck $p_{\mathrm{g}}$ des Gases in der Blase werden als homogen angenommen.

Im Gegensatz zum Eick Modell basiert das Toegel Modell [24] auf dem Keller-Miksis Model[ $[1$ und beschreibt zusätzlich den Stofftransport über die Blasenwand durch Phasenübergänge, zumindest näherungsweise. Hier gibt es für die Stoffmenge $N$ der Wassermoleküle in der Blase und für die Temperatur $T$ jeweils eine zusätzliche gewöhnliche Differentialgleichung. Das vollständige Differentialgleichungssystem lautet:

\footnotetext{
${ }^{1}$ Ein vergleichbares Modell lässt sich prinzipiell auch auf dem Gilmore Modell aufbauen.
} 


$$
\begin{aligned}
\left(1-\frac{\dot{R}}{c_{0}}\right) R \ddot{R}+\frac{3}{2} \dot{R}^{2}\left(1-\frac{\dot{R}}{3 c_{0}}\right)= & \left(1+\frac{\dot{R}}{c_{0}}\right) \frac{1}{\varrho}\left(p_{\mathrm{g}}-p_{\text {ext }}(t)-p_{\text {stat }}\right)-\frac{2 \sigma}{\varrho R} \\
& +\frac{R}{\varrho c_{0}}\left(\dot{p}_{\mathrm{g}}-\dot{p}_{\text {ext }}(t)\right)-4 \mu \frac{\dot{R}}{\varrho R}-4 \mu \frac{\ddot{R}}{\varrho c_{0}} \\
\dot{N}= & 4 \pi R^{2} D \frac{n_{R}-n}{l_{\text {diff }}}=S D \frac{n_{R}-n}{l_{\text {diff }}} \\
\dot{T}= & \frac{\dot{Q}}{C_{V}}-\frac{p_{g} \dot{V}}{C_{V}}+\left(4 T_{1}-3 T\right. \\
& \left.-T \sum \frac{\theta_{i} / T}{e^{\theta_{i} / T}-1}\right) \frac{\dot{N} R_{\mathrm{g}}}{C_{V}}
\end{aligned}
$$

Da hier frei schwingende Blasen modelliert werden sollen, wird $p_{\text {ext }}(t)=0$ gesetzt. Für eine akustische Anregung kann $p_{\text {ext }}(t)$ wie in Gl. (2.5) gewählt werden.

Zur Integration der Gleichungen 2.17) -2.19) werden in jedem Zeitschritt folgende Hilfsgrößen berechnet:

$$
\begin{aligned}
S & =4 \pi R^{2} \\
V & =\frac{4 \pi R^{3}}{3} \\
\dot{V} & =4 \pi R^{2} \dot{R}=S \dot{R} \\
n & =N / V
\end{aligned}
$$

Der Druck in der Blase wird durch eine Van-der-Waals Gleichung festgelegt, in der der Binnendruck nicht berücksichtigt wird:

$$
\begin{aligned}
p_{\mathrm{g}}= & \frac{\left(N+N_{\mathrm{g}}\right) R_{\mathrm{g}} T}{\frac{4 \pi}{3}\left(R^{3}-\left(R_{\mathrm{n}} / a\right)^{3}\right)} \\
\dot{p}_{\mathrm{g}}= & \frac{R_{\mathrm{g}}}{\frac{4 \pi}{3}\left(R^{3}-\left(R_{\mathrm{n}} / a\right)^{3}\right)}\left(\dot{N} T+N \dot{T}+N_{\mathrm{g}} \dot{T}\right) \\
& -\frac{R_{\mathrm{g}}}{\frac{4 \pi}{3}}\left(N+N_{\mathrm{g}}\right) T \frac{3 R^{2} \dot{R}}{\left(R^{3}-\left(R_{\mathrm{n}} / a\right)^{3}\right)^{2}}
\end{aligned}
$$

mit dem Van-der-Waals Parameter $a$, durch den ein minimaler Radius (hard-core radius) der Blase festgelegt wird. Hier wird der Wert $a=11.63$ für Wasserstoff verwendet. Toegel et al. [24] wählen $a=8.86$. 
Stofftransport und Phasenübergänge

Die Stoffmenge der Gasmoleküle ergibt sich im Druckgleichgewicht mit dem vorgegebenen Gleichgewichtsradius $R_{\mathrm{n}, \mathrm{g}}$ aus der idealen Gasgleichung:

$$
N_{\mathrm{g}}=\frac{1}{R_{\mathrm{g}} T_{1}} \frac{4 \pi}{3} R_{\mathrm{n}, \mathrm{g}}^{3}\left(p_{\mathrm{stat}}+\frac{2 \sigma}{R_{\mathrm{n}, \mathrm{g}}}\right)
$$

Diese Stoffmenge bleibt für die gesamte Rechnung gleich, die Diffusion von Gas durch die Blasenwand wird also vernachlässigt.

Der Diffusionskoeffizient $D$ in $\mathrm{cm}^{2} \mathrm{~s}^{-1}$ für eine Mischung aus zwei Stoffen (hier Wasserdampf und ein Gas) lässt sich nach [25], Gl. (8.2-44) und [26], Gl. (17.3-11) wie folgt berechnen:

$$
D=2.2646 \cdot 10^{-5} \sqrt{T\left(\frac{1}{M_{\mathrm{v}}}+\frac{1}{M_{\mathrm{g}}}\right)} \frac{1}{\sigma_{\mathrm{vg}}^{2} \Omega_{\mathrm{vg}}\left(n_{R}+N_{\mathrm{g}} / V\right)}
$$

Dabei ist $T$ die Temperatur in $\mathrm{K}, M_{\mathrm{V}}$ und $M_{\mathrm{g}}$ sind die Molmassen des Dampfs bzw. des Gases in der Blase in $\mathrm{g} \mathrm{mol}^{-1}$. Das Kollisionsintegral für Diffusion $\Omega_{\mathrm{vg}}=\Omega_{\mathrm{vg}}\left(k_{\mathrm{B}} T / \varepsilon_{\mathrm{vg}}\right)$ ist dimensionslos. Die Parameter $\sigma_{\mathrm{vg}}[\AA]$ und $\varepsilon_{\mathrm{vg}}[\mathrm{J}]$ bestimmen die Form des LennardJones Potentials der beiden Molekülsorten [26]:

$$
\varphi_{\mathrm{vg}}(r)=4 \varepsilon_{\mathrm{vg}}\left[\left(\frac{\sigma_{\mathrm{vg}}}{r}\right)^{12}-\left(\frac{\sigma_{\mathrm{vg}}}{r}\right)^{6}\right]
$$

Die Diffusionslänge wird in jedem Zeitschritt neu bestimmt und ist nach oben durch den Wert $R / \pi$ begrenzt:

$$
l_{\mathrm{diff}}=\min (\sqrt{D R / \dot{R}}, R / \pi)
$$

Die Stoffmengendichte im Temperaturgleichgewicht $T=T_{1}$ ist

$$
n_{R}=\frac{p_{\mathrm{v}}\left(T_{1}\right)}{R_{\mathrm{g}} T_{1}}
$$

und ergibt sich aus dem Dampfdruck $p_{\mathrm{v}}\left(T_{1}\right)$, der mit Hilfe einer Anpassung aus [27] bestimmt wird (siehe auch Abb. 2.3).

Der Ruheradius $R_{\mathrm{n}}$ hängt über die Van-der-Waals Beziehung in Gl. (2.24) mit der Stoffmenge $N$ zusammen. Im Gleichgewicht gilt:

$$
\left(p_{\text {stat }}+\frac{2 \sigma}{R_{\mathrm{n}}}\right) \cdot \frac{4 \pi}{3}\left(R_{\mathrm{n}}^{3}-\left(R_{\mathrm{n}} / a\right)^{3}\right)=\left(N+N_{\mathrm{g}}\right) R_{\mathrm{g}} T_{1}
$$


Der Ruheradius $R_{\mathrm{n}}$ lässt sich also bei gegebener Stoffmenge durch Bestimmung einer Nullstelle der Funktion

$$
\begin{aligned}
f\left(R_{\mathrm{n}}\right)= & p_{\mathrm{stat}}\left(1-1 / a^{3}\right) R_{\mathrm{n}}^{3} \\
& +2 \sigma\left(1-1 / a^{3}\right) R_{\mathrm{n}}^{2} \\
& -\frac{3}{4 \pi}\left(N+N_{\mathrm{g}}\right) R_{\mathrm{g}} T_{1}
\end{aligned}
$$

ermitteln. Die Funktion $f\left(R_{\mathrm{n}}\right)$ hat nur eine einzige Nullstelle, wie das Beispiel in Abb. 2.2 zeigt.

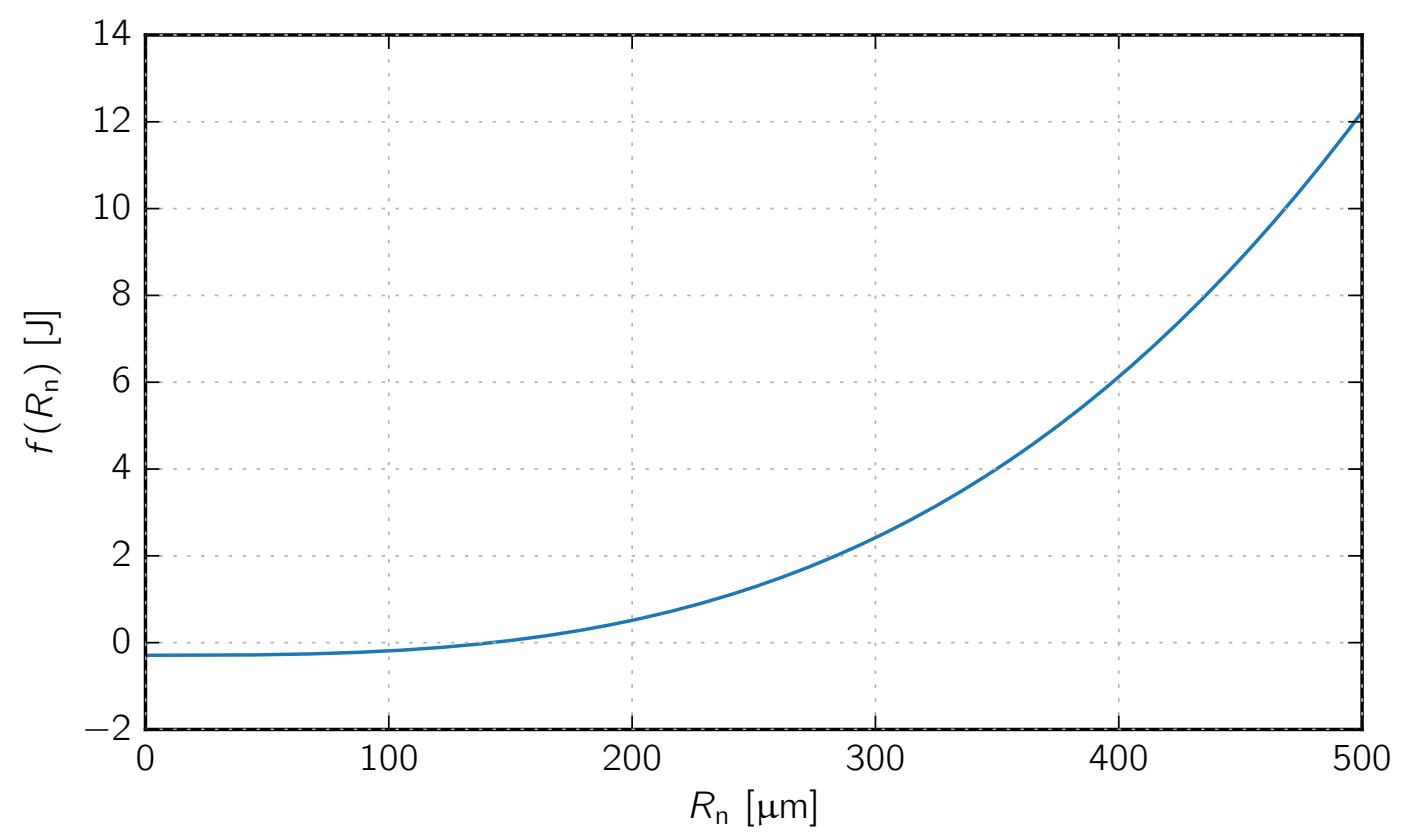

Abbildung 2.2: Die Funktion $f\left(R_{\mathrm{n}}\right)$ aus Gl. 2.32) für $p_{\text {stat }}=1$ bar, $T_{1}=20^{\circ} \mathrm{C}, \sigma=$ $0.07 \mathrm{~N} \mathrm{~m}^{-1}, N=7.3 \cdot 10^{-9}$ mol und $N_{\mathrm{g}}=3.0 \cdot 10^{-13} \mathrm{~mol}$.

\section{Wärmetransport}

Für die Temperaturleitfähigkeit des Gas-/Dampfgemisches in der Blase wird hier angenommen:

$$
\alpha=\frac{k_{\text {mix }}}{\varrho_{\text {mix }} c_{p, \text { mix }}}=\frac{k_{\text {mix }}}{4 n_{R} R_{\mathrm{g}}+\frac{5}{2} N_{\mathrm{g}} R_{\mathrm{g}} / V}
$$

Da sich während der Rechnung in Gl. (2.33) nur das Blasenvolumen $V$ ändern kann, ist die Temperaturleitfähigkeit hier nur von $V$ und damit von der Stoffmengendichte $N_{\mathrm{g}} / V$ 
abhängig. Werte der spezifischen Wärmekapazität $c_{p}$ für verschiedene Stoffe sind in [28] angegeben (z. B. Wasserstoff: $c_{p}=3.5 R_{\mathrm{g}}$ ).

Die thermische Diffusionslänge

$$
l_{\mathrm{th}}=\min (\sqrt{\alpha R / \dot{R}}, R / \pi)
$$

wird in jedem Zeitschritt neu berechnet und ist nach oben durch $R / \pi$ begrenzt. Der Term

$$
\dot{Q}=4 \pi R^{2} k_{\text {mix }} \frac{T_{1}-T}{l_{\text {th }}}
$$

in Gl. (2.19) beschreibt näherungsweise die Wärmeleitung über die Blasenwand. Die Ableitung der Temperatur nach dem Ort (siehe Gl. (2.37) wurde hier durch den Quotienten $\left(T_{1}-T\right) / l_{\text {th }}$ ersetzt. Außerdem tritt in Gl. (2.19) die Wärmekapazität $C_{V}$ des Gas-/Dampfgemisches in der Blase auf, die hier wie folgt berechnet wird:

$$
C_{V}=\frac{3}{2} N_{\mathrm{g}} R_{\mathrm{g}}+\left(3+\sum \frac{\left(\theta_{i} / T\right)^{2} e^{\theta_{i} / T}}{\left(e^{\theta_{i} / T}-1\right)^{2}}\right) N R_{\mathrm{g}}
$$

Die $\theta_{i}$ in Gl. (2.19) und (2.36) sind die Temperaturen der Schwingungsfreiheitsgrade der Wassermoleküle [29].

Die Werte von Schallgeschwindigkeit $c_{0}$, Dichte $\varrho$, Viskosität $\mu$ und Dampfdruck $p_{\mathrm{v}}$ hängen von der verwendeten Flüssigkeit ab (hier Wasser). Die folgenden Größen richten sich nach der Kombination aus Flüssigkeit, Dampf und Gas: Oberflächenspannung $\sigma$, Diffusionskoeffizient $D$, Temperaturleitfähigkeit $\alpha$ und Wärmeleitfähigkeit $k$, Wärmekapazität bei konstantem Volumen $C_{V}$ und spezifische Wärmekapazität bei konstantem Druck $c_{p}$, Van-der-Waals Parameter (Kovolumen/Radius) $a$.

Neben den bisher beschriebenen Blasenmodellen gibt es noch einige andere, die aber komplexer sind und damit zu aufwändigeren Rechnungen führen. Ein weiteres Modell, das neben der Kompressibilität der Flüssigkeit auch Phasenübergänge und Wärmeleitung berücksichtigt, stammt von Dreyer et al. [30]. In diesem Modell treten neben gewöhnlichen Differentialgleichungen auch partielle Differentialgleichungen und DelayDifferentialgleichungen auf, so dass die numerische Lösung wesentlich aufwändiger ist.

Es besteht auch die Möglichkeit der numerischen Lösung von Kontinuitäts- und Impulsgleichung. Die dabei auftretenden partiellen Differentialgleichungen lassen sich z. B. mit OpenFOAM lösen [31, 18]. Zur Berücksichtigung der Temperatur muss zusätzlich eine Energiegleichung eingeführt werden. 
Die Molekulardynamiksimulationen einer kollabierenden Blase von Schanz et al. [32] liefern Temperatur, Dichte und Druck innerhalb der Blase, jeweils orts- und zeitaufgelöst. Da die Rechnungen sehr aufwändig sind, bekommt man aber immer nur Ergebnisse für ein sehr kleines Zeitfenster (etwa $1 \mathrm{~ns}$ ).

\subsection{Blasendynamik in Wasser bei erhöhten Temperaturen}

Die Dynamik von Blasen hängt stark von der Flüssigkeit ab, in der sie sich befinden. Vor allem für das in Kap. 3 beschriebene Experiment sind daher die Eigenschaften von Wasser und insbesondere ihre Temperaturabhängigkeit von entscheidender Bedeutung.

Die in Abschnitt 2.1 besprochenen Blasenmodelle berücksichtigen den Dampfdruck $p_{\mathrm{v}}$, die Viskosität $\mu$ und die Oberflächenspannung $\sigma$. Die Schallabstrahlung von der schwingenden Blase ist im Rayleigh-Plesset Modell nicht enthalten; in allen anderen Modellen tritt die Schallgeschwindigkeit $c$ als zusätzlicher Parameter auf.

Die Temperaturabhängigkeit kann nun durch Einsetzen der Werte für $p_{\mathrm{v}}, \mu, \sigma$ und $c$ bei der entsprechenden Temperatur beschrieben werden. Es stellt sich aber die Frage, welche dieser Größen eine starke Temperaturabhängigkeit aufweisen und damit für die Blasendynamik besonders wichtig sind. Diese Temperaturabhängigkeit der genannten Größen gilt nur im Gleichgewicht. Darüber hinaus können aber während der Blasenschwingung auch Nichtgleichgewichtszustände auftreten.

Bei einer Erhöhung der Temperatur von Wasser ändert sich vor allem der Dampfdruck; dieser steigt exponentiell an (Abb. 2.3). Zunächst sind die Änderungen klein, aber mit zunehmender Temperatur steigt der Dampfdruck immer stärker an. Diese Temperaturabhängigkeit des Dampfdrucks ist wesentlich für die Blasendynamik. Die Viskosität der Flüssigkeit (Abb. 2.3) spielt vor allem bei kleinen Blasen sowie bei hohen Blasenwandgeschwindigkeiten (also im Kollaps) eine Rolle, weil dann der Term $4 \mu \dot{R} / R$ betragsmäßig groß wird. Bei sehr kleinen Blasen ist auch der Krümmungsradius der Oberfläche sehr klein und der Einfluss der Oberflächenspannung (Abb. 2.4) nimmt zu. Der Term $2 \sigma / R$ wird in diesem Fall betragsmäßig groß. Die Schallgeschwindigkeit von Wasser (Abb. 2.4) steigt bis zu einer Temperatur von etwa $72^{\circ} \mathrm{C}$ an und nimmt oberhalb davon wieder ab. Der Einfluss dieser Temperaturabhängigkeit auf die Blasendynamik ist meist vernachlässigbar. 

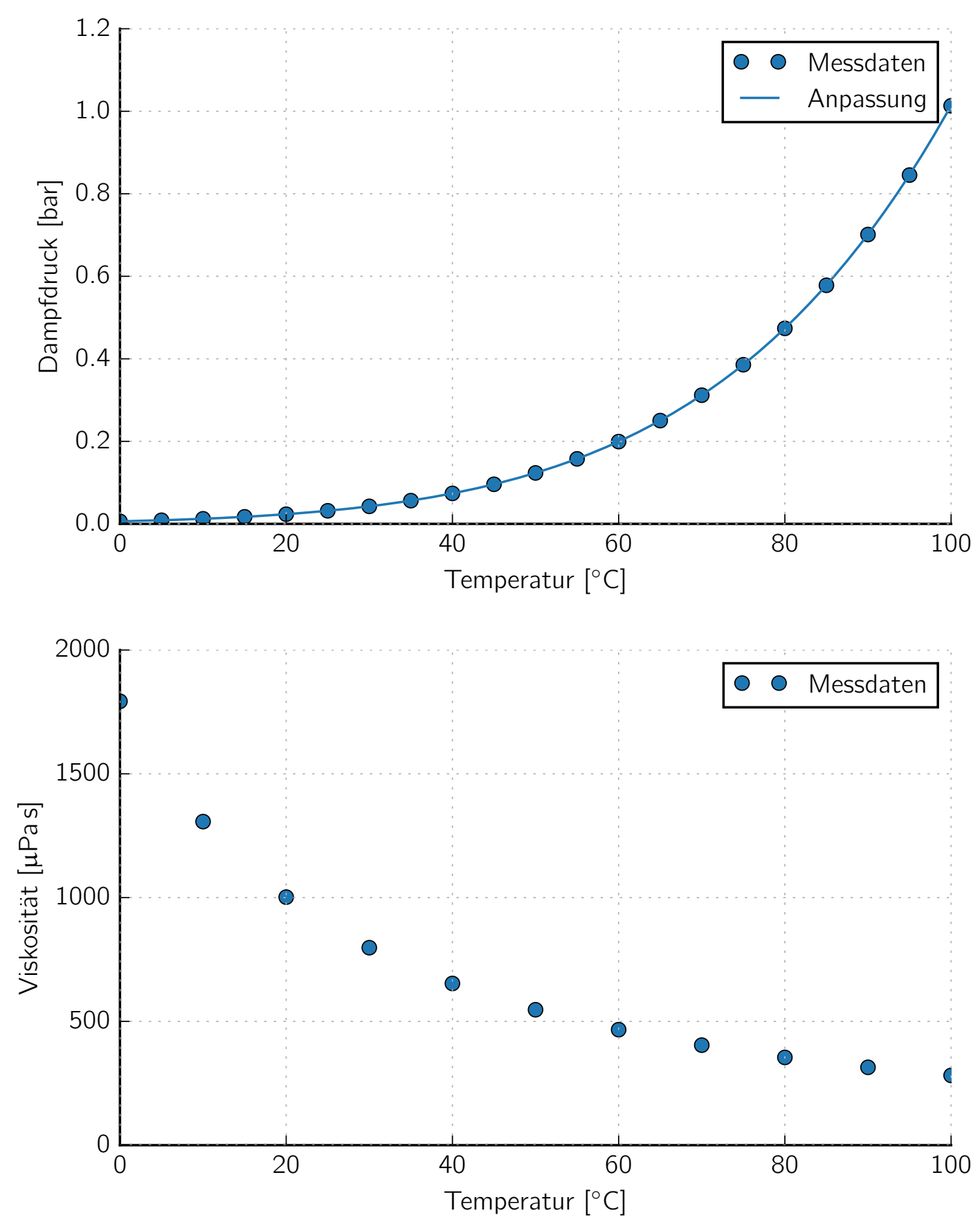

Abbildung 2.3: Dampfdruck von Wasser als Funktion der Temperatur (oben, Messdaten entnommen aus [33], Anpassung aus [27]) und Viskosität von Wasser als Funktion der Temperatur (unten, Messdaten entnommen aus [34]). 

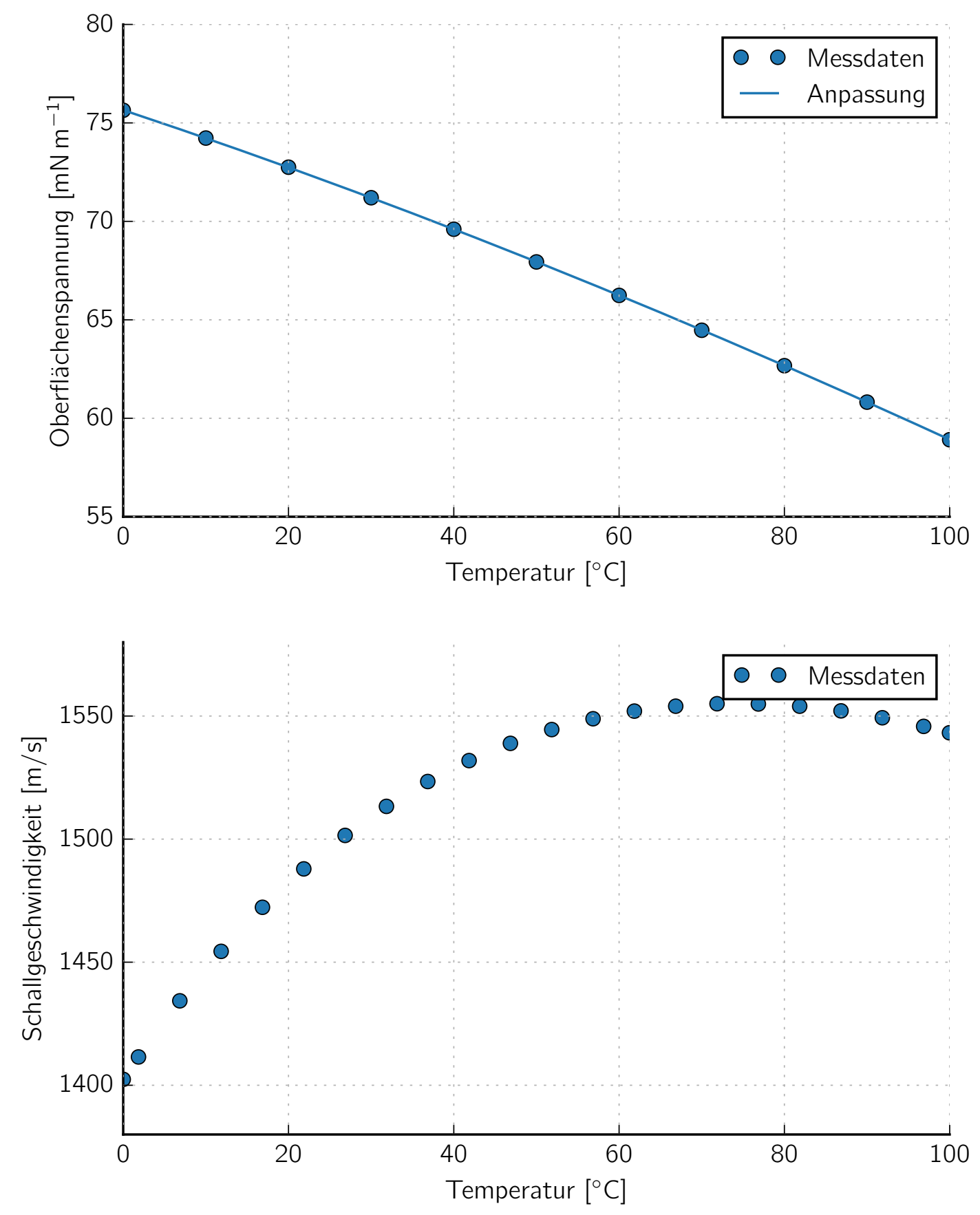

Abbildung 2.4: Oberflächenspannung von Wasser als Funktion der Temperatur (oben, Messdaten entnommen aus [34], Anpassung aus [35]). Schallgeschwindigkeit von Wasser bei verschiedenen Temperaturen (unten, Messdaten entnommen aus [27]). 


\subsection{Wärmetransport über die Blasenwand}

Grundsätzlich kann Wärmetransport auf drei verschiedene Arten erfolgen: durch Wärmeleitung, Konvektion und Strahlung. Im Fall von Kavitationsblasen muss man unterscheiden zwischen dem Wärmetransport innerhalb der Blase (also im Gas-/Dampfgemisch) und dem Wärmetransport in der Flüssigkeit rund um die Blase.

Im Kollaps steigt die Temperatur vor allem im Zentrum der Blase an. Der dadurch entstehende Temperaturgradient führt zu einem Wärmestrom vom Zentrum zur Blasenwand. Nun kann die Wärme auch in der Flüssigkeit transportiert werden, wobei hier angenommen wird, dass dies vor allem durch Wärmeleitung geschieht. Bei einem schnellen Blasenkollaps und besonders beim asphärischen Kollaps mit Jetbildung kann in Folge der Strömung auch Wärmetransport durch Konvektion stattfinden.

Die Wärmeleitungsgleichung lautet [26, 28]:

$$
\frac{\partial T}{\partial t}=\frac{k}{\varrho c_{p}} \nabla^{2} T=\alpha \nabla^{2} T
$$

Sie hat die gleiche Struktur wie die Diffusionsgleichung. Hier treten als Parameter die Wärmeleitfähigkeit $k$ und die Temperaturleitfähigkeit $\alpha$ auf, die wie folgt zusammenhängen:

$$
\alpha=\frac{k}{\varrho c_{p}}
$$

Wenn auch Wärmetransport durch Konvektion berücksichtigt werden soll, kann dazu ein zusätzlicher Term $\vec{v} \cdot \nabla T$ eingeführt werden [26]:

$$
\frac{\partial T}{\partial t}+\vec{v} \cdot \nabla T=\frac{k}{\varrho c_{p}} \nabla^{2} T
$$

Die Wärmeleitungsgleichung 2.37) ist eine parabolische Differentialgleichung und kann u. a. durch Fourieranalyse oder mit der Methode der finiten Differenzen gelöst werden [36].

Für die Temperaturleitfähigkeit im Blaseninneren wird $\alpha=20 \cdot 10^{-6} \mathrm{~m}^{2} \mathrm{~s}^{-1}$ (Luft) angenommen und in der Flüssigkeit $\alpha=0.143 \cdot 10^{-6} \mathrm{~m}^{2} \mathrm{~s}^{-1}$ (Wasser). Geht man von einer Blase mit dem Radius $R=100 \mu \mathrm{m}$ aus und von einer homogenen Anfangstemperatur von $T=100^{\circ} \mathrm{C}$ innerhalb der Blase, so ergeben sich in einer Flüssigkeit der Temperatur $T=20^{\circ} \mathrm{C}$ die in Abb. 2.5 dargestellten Temperaturverteilungen. 


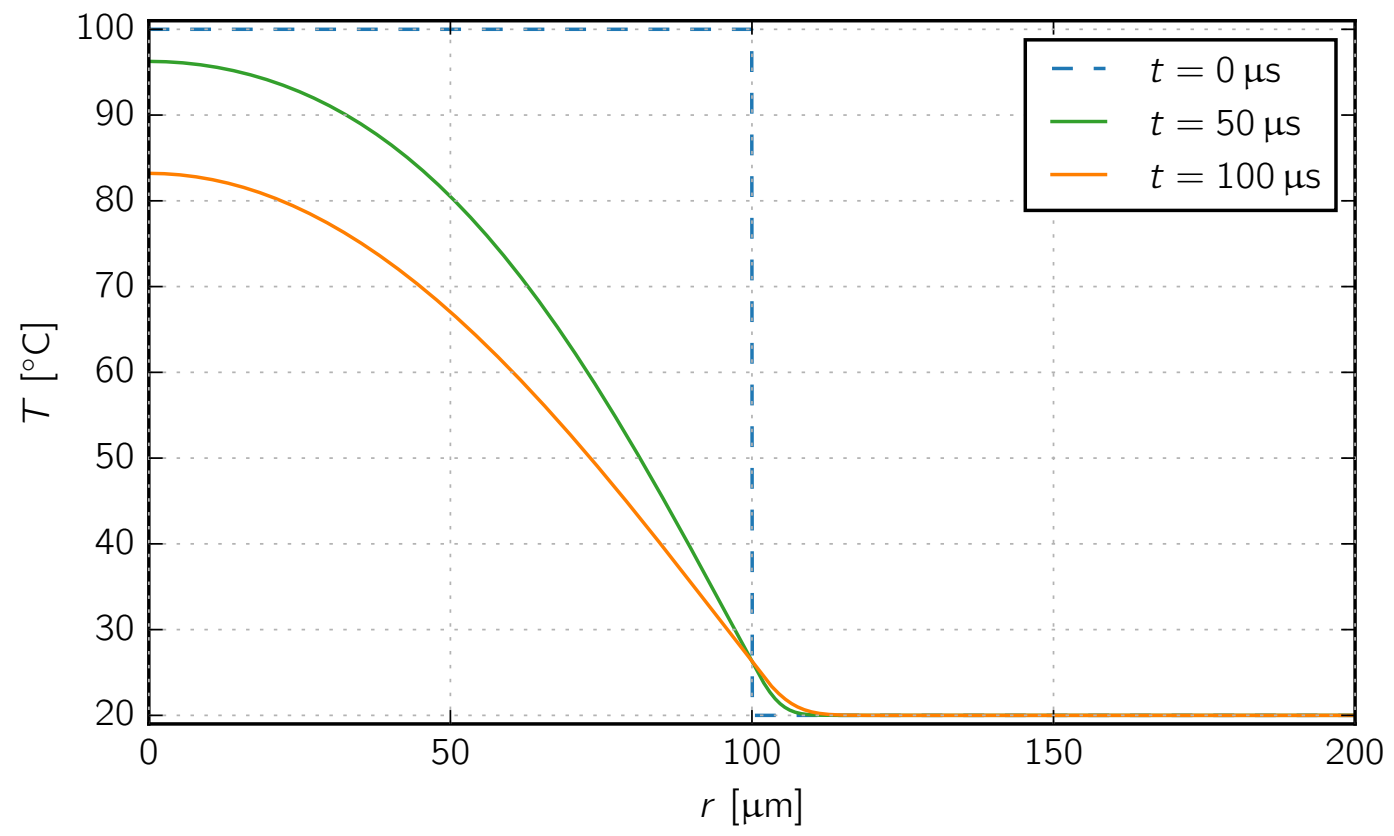

Abbildung 2.5: Numerische Lösung der Wärmeleitungsgleichung mit der Methode der finiten Differenzen. Im Blaseninneren (links) gilt $\alpha=20 \cdot 10^{-6} \mathrm{~m}^{2} \mathrm{~s}^{-1}$ (Luft), in der Flüssigkeit (rechts) ist $\alpha=0.143 \cdot 10^{-6} \mathrm{~m}^{2} \mathrm{~s}^{-1}$ (Wasser).

\subsection{Lasererzeugte Kavitation}

Für die Erzeugung von einzelnen Blasen im Experiment kann man einen gepulsten Laser verwenden. Ein kurzer Laserpuls wird in eine Flüssigkeit (z. B. Wasser) fokussiert, es kommt zum optischen Durchbruch und eine Blase entsteht [10, 37]. Ein Vorteil dieser Methode ist, dass man im Verlauf des Experiments viele gleiche Blasen reproduzierbar erzeugen kann. Diese Form der Kavitation wird als optische Kavitation oder lasererzeugte Kavitation bezeichnet.

Für einen optischen Durchbruch in transparenten Flüssigkeiten (z. B. Wasser) sind Laserpulse mit ausreichend hoher Pulsenergie notwendig [37]. Dann werden die Wassermoleküle durch das Laserlicht ionisiert und bilden zusammen mit den entstandenen freien Elektronen ein Plasma. Die freien Elektronen geben durch Stöße und Rekombinationen Energie an die Wassermoleküle ab. Dadurch steigen Druck und Temperatur stark an. Es wird eine Stoßwelle ausgesandt und eine Blase bildet sich, die aufgrund 


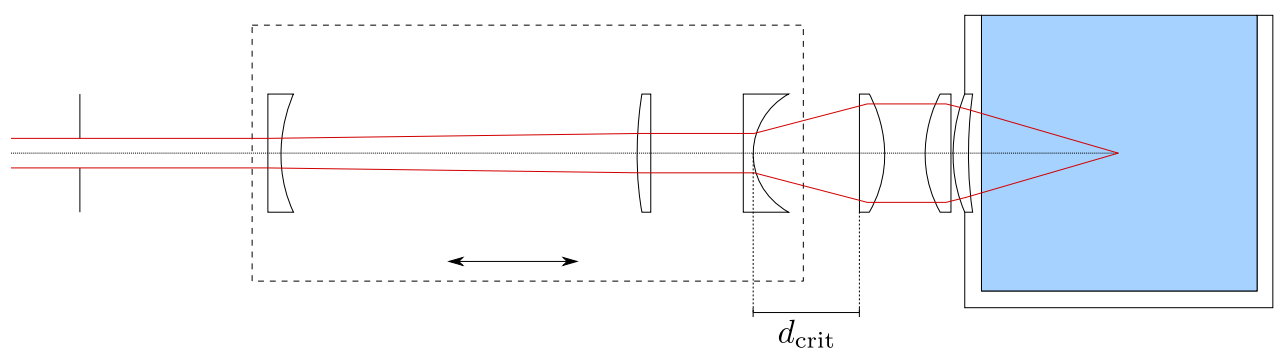

Abbildung 2.6: Linsensystem zur Fokussierung eines Laserpulses in eine Küvette. Die ersten drei Linsen sind als Einheit verschiebbar, so dass der Abstand $d_{\text {crit }}$ präzise eingestellt werden kann.

des hohen Drucks im Inneren zunächst anwächst.

Die Form der entstehenden Blasen hängt von Wellenlänge, Pulsdauer und Pulsenergie des Lasers sowie von der Fokussierungsoptik (z. B. von deren numerischer Apertur) ab. Außerdem verschiebt sich mit der Dauer der Laserpulse die Durchbruchschwelle [38].

Nachdem von Lauterborn [7] und anderen [37] um 1970 herum erstmals Einzelblasen mit gepulsten Lasern erzeugt wurden, folgten zahlreiche weitere Veröffentlichungen über lasererzeugte Blasen. Untersucht wurden u. a. die Kavitationserosion durch einzelne Blasen [2], die Dynamik von Nanosekunden- und Femtosekundenlaserblasen [39], lasererzeugte Blasen im Ultraschallfeld [40] sowie das Strömungsfeld um eine einzelne Blase [41]. Außerdem wurden Experimente zur Wechselwirkung zweier lasererzeugter Blasen [31], zum Einfluss der Viskosität [42] sowie der Gravitation [43] auf die Blasendynamik durchgeführt.

Akhatov et al. [44] stellen ein Modell für eine kugelförmige lasererzeugte Blase vor und vergleichen die Ergebnisse der numerischen Rechnungen mit gemessenen Blasenradien. Köhler [45] hat numerische Untersuchungen zum optischen Durchbruch durchgeführt.

\subsubsection{Blasenerzeugung im Experiment mit gepulsten Lasern}

Da in den Experimenten der vorliegenden Arbeit häufig von der lasererzeugten Kavitation Gebrauch gemacht wird, um einzelne Blasen zu erzeugen, soll deren experimentelle Realisierung hier kurz beschrieben werden. 
Ein Nd:YAG-Laser liefert Pulse mit einer Dauer von wenigen Nanosekunden, die in eine mit Wasser gefüllte Küvette fokussiert werden. Dazu dient ein speziell entwickeltes Linsensystem (Abb. 2.6), das auf minimale sphärische Aberrationen optimiert wurde um einen möglichst kleinen Fokus und damit sphärische Blasen zu erhalten. Mit der Software WinLens [46] der Firma qioptiq wurde eine Strahlverfolgung (raytracing) durchgeführt und das Linsensystem optimiert. Das hier verwendete Linsensystem ist ähnlich aufgebaut wie die von Geisler entwickelten Systeme [39].

Der Laserstrahl wird zunächst aufgeweitet und dann in die Küvette fokussiert. Auf diese Weise kann bei gleicher Position des Fokus in der Küvette (also bei gleicher Brennweite) in einem größeren Winkel fokussiert werden. Es ergibt sich eine höhere numerische Apertur und ein enger begrenzter Fokus. Zur Minimierung der sphärischen Aberrationen muss der Abstand $d_{\text {crit }}$ in Abb. 2.6 sehr genau (auf ca. $20 \mu \mathrm{m}$ ) eingestellt werden. Daher sind die ersten drei Linsen auf einem Verschiebetisch montiert und lassen sich mit einer Mikrometerschraube in Richtung der optischen Achse verschieben.

Es werden in den Experimenten verschiedene Küvetten und Laser verwendet. Dadurch wird jeweils eine Anpassung des Linsensystems notwendig. Beim Wechsel zu einer Küvette anderer Größe wird lediglich die letzte Linse vor der Küvette durch eine andere ersetzt, so dass der Fokus in der Mitte der Küvette liegt. Soll ein Laser mit anderer Wellenlänge zur Blasenerzeugung verwendet werden, müssen mehrere Linsen ausgetauscht werden um die sphärischen Aberrationen zu minimieren. Der prinzipielle Aufbau des Linsensystems bleibt aber gleich. Im Anhang B sind die verschiedenen Linsensysteme und die darin verwendeten Linsen aufgeführt.

Ein $\lambda / 2$-Plättchen vor einem polarisierenden Strahlteilerwürfel dient zur Einstellung der Pulsenergie. So kann der Laser bei hoher Leistung betrieben werden und liefert ein besseres Strahlprofil sowie eine stabilere Energie von Puls zu Puls. Mit dem Strahlteiler wird überschüssiges Laserlicht in einen Strahlsumpf (beam dump) gelenkt (siehe Abb. 3.1.

\subsection{Akustische Blasenfallen und Sonochemie}

Im Druckbauch eines stehenden Schallwellenfeldes kann eine einzelne Blase eingefangen und stabil gehalten werden [10]. Die Blase wird durch das angelegte Schallfeld zu Schwingungen angeregt und emittiert unter bestimmten Bedingungen im Kollaps einen Lichtpuls. Dies wird als Einzelblasen-Sonolumineszenz (single bubble sonoluminescence, SBSL) bezeichnet und wurde erstmals von Gaitan et al. [47] im Experiment erreicht. Ei- 
ne gute Übersicht zum Thema Sonolumineszenz bieten die Artikel von Crum [48] und Brenner et al. [49].

Das Einfangen einer Blase in einer akustischen Falle ist neben der lasererzeugten Kavitation eine weitere hilfreiche Methode zur experimentellen Untersuchung von einzelnen Blasen. Für eine akustische Blasenfalle wird meist eine Küvette aus Glas verwendet, an deren Unterseite eine Piezoscheibe geklebt ist. Letztere dient als Ultraschallwandler (transducer) und erzeugt ein stehendes Schallwellenfeld in der Küvette. Damit die eingefangene Blase über einen längeren Zeitraum stabil schwingt, ist es erforderlich, dass ihre Position, ihre Form und der Gasgehalt im Inneren stabil ist [10]. Folgende Parameter haben einen wesentlichen Einfluss auf die Blasendynamik: Frequenz und Amplitude des Anregungssignals, statischer Druck, Gasgehalt der Flüssigkeit, Füllhöhe in der Küvette, Temperatur. In diesem Parameterraum wurden u. a. von Koch et al. [50] durch numerische Rechnungen Bereiche bestimmt, in denen eine Blase stabil schwingt - die „Lebensräume“ der Blase.

Im Schallfeld schwingende Blasen können chemische Reaktionen beschleunigen, da im Kollaps kurzzeitig sehr hohe Drücke und Temperaturen erreicht werden. Dies wird als Sonochemie bezeichnet und es können dabei auch Reaktionsprodukte entstehen, die sich sonst nur aufwändig oder gar nicht herstellen lassen [51]. Während in den von Suslick [51] beschriebenen Experimenten viele Blasen an den sonochemischen Reaktionen beteiligt sind, berichten Lepoint et al. [52], dass auch einzelne Blasen im Schallfeld chemisch aktiv sind. Die sonochemischen Reaktionsprodukte können auch dazu dienen, Strömungen in Flüssigkeiten sichtbar zu machen, z. B. die Strömung in der Nähe einer SBSL-Blase [53]. 



\section{Dynamik lasererzeugter Kavitationsblasen bei verschiedenen Wassertemperaturen}

In diesem Kapitel wird der Einfluss der Wassertemperatur auf die Dynamik von frei schwingenden sphärischen Blasen untersucht. Dies ist überall dort relevant, wo Blasen in Flüssigkeiten hoher Temperatur auftreten, z. B. in Kühlsystemen. Im Experiment werden lasererzeugte Blasen bei verschiedenen Wassertemperaturen im Bereich von $10^{\circ} \mathrm{C}$ bis $80^{\circ} \mathrm{C}$ erzeugt und ihre Dynamik mit einer Hochgeschwindigkeitskamera aufgezeichnet. Zum Vergleich werden die Blasenschwingungen mit verschiedenen Modellen numerisch berechnet.

In Experimenten zur Sonolumineszenz von einzelnen Blasen (single bubble sonoluminescence, SBSL) wurden vor allem Blasen bei niedrigeren Temperaturen untersucht [54]. Beim Absenken der Temperatur wurde ein Anstieg in der Intensität der Sonolumineszenz beobachtet.

Ebeling [55] sowie Hentschel und Lauterborn [56] haben Experimente mit lasererzeugten Blasen in Wasser bei Raumtemperatur und numerische Rechnungen mit dem Gilmore Modell durchgeführt. In beiden Arbeiten werden Kondensation und Diffusion durch Änderung des Ruheradius während der Rechnung berücksichtigt.

Die Dynamik von lasererzeugten Blasen in Wasser bei Temperaturen im Bereich von $10^{\circ} \mathrm{C}$ bis $35^{\circ} \mathrm{C}$ wurde von Eick [20] und Koch et al. [57] experimentell untersucht. In [20] wird auch ein Modell zur Beschreibung der Blasendynamik bei verschiedenen Wassertemperaturen vorgestellt, das auf dem Gilmore Modell basiert (siehe Abschnitt 3.2.2).

Theoretische Untersuchungen zur Temperaturabhängigkeit der Dynamik von einzelnen frei schwingenden Blasen in Wasser wurden von Nigmatulin und Khabeev [22], Prosperetti et al. [23], Eick [20] und Hegedus et al. [42] durchgeführt.

Toegel et al. [24] (siehe auch Abschnitt 3.2.3), Storey und Szeri [58] sowie Stricker et al. [59] haben die Dynamik von SBSL-Blasen, also einzelnen Blasen im Schallfeld, numerisch untersucht. 
Ein vergleichsweise einfaches Modell für eine frei schwingende Blase in Wasser, das Kompressibilität, Phasenübergänge und Wärmeleitung berücksichtigt, haben Dreyer et al. [30] vorgestellt.

Genauere, aber auch aufwändiger zu lösende, Modelle basierend auf partiellen Differentialgleichungen (PDEs) wurden von Fujikawa und Akamatsu [8], Akhatov et al. [44], Fuster et al. [17], Han et al. [31] und Koch et al. [18] aufgestellt und numerisch gelöst.

Die genannten Arbeiten berücksichtigen bei der Modellierung einzelne oder mehrere der folgenden Effekte: Kompressibilität der Flüssigkeit, Phasenübergänge, Wärmeleitung, Diffusion. Es stellt sich nun die Frage, welche dieser Effekte für die Blasendynamik bei hohen Temperaturen wichtig sind.

Bislang gibt es nur wenige Veröffentlichungen zu Experimenten mit Blasen bei hohen Wassertemperaturen [20, 57]. Im Vergleich zu früheren Arbeiten wird in diesem Experiment ein größerer Temperaturbereich abgedeckt und dieser feiner abgetastet.

\subsection{Experiment}

Mit Hilfe von Nanosekundenlaserpulsen werden, wie im Abschnitt 2.4.1 beschrieben, einzelne Blasen in Wasser erzeugt. Ein Thermostat regelt die Wassertemperatur. Wie bereits in Abschnitt 2.2 angesprochen wurde, ändert sich bei steigender Wassertemperatur vor allem der Dampfdruck (siehe Abb. 2.3). In diesem Experiment soll untersucht werden, wie sich das auf die Blasendynamik auswirkt.

Der experimentelle Aufbau ist in Abb. 3.1 schematisch dargestellt. Für das Experiment hat die feinmechanische Werkstatt des Dritten Physikalischen Instituts eine spezielle Küvette aus Makrolon (Polycarbonat) angefertigt. Im Vergleich zu Plexiglas (PMMA), das schon für andere Küvetten verwendet wurde, bleibt dieses Material auch bei höheren Temperaturen fest und formstabil. Im Innern befindet sich eine weitere Küvette aus Glas (Innenabmessungen: $40 \mathrm{~mm} \times 40 \mathrm{~mm} \times 40 \mathrm{~mm}$ ), die durch einen Teflondeckel abgeschlossen ist. Der Deckel besitzt Aussparungen für ein Hydrofon, einen Temperatursensor (Pt100) sowie für einen kleinen Schlauch zur Befüllung. Die innere Glasküvette wird mit sauberem deionisierten Wasser gefüllt. In der äußeren Küvette zirkuliert Wasser (ebenfalls deionisiert) aus einem Thermostaten (Kühlwasserbad B. Braun Frigomix U mit Thermostat Lauda E200). Auf diese Weise kann die Temperatur des Wassers in der Glasküvette geregelt werden. Die zusätzliche Glasküvette sorgt dafür, dass das Wasser im Inneren sauber bleibt und dort von außen keine Strömungen hervorgerufen werden. 


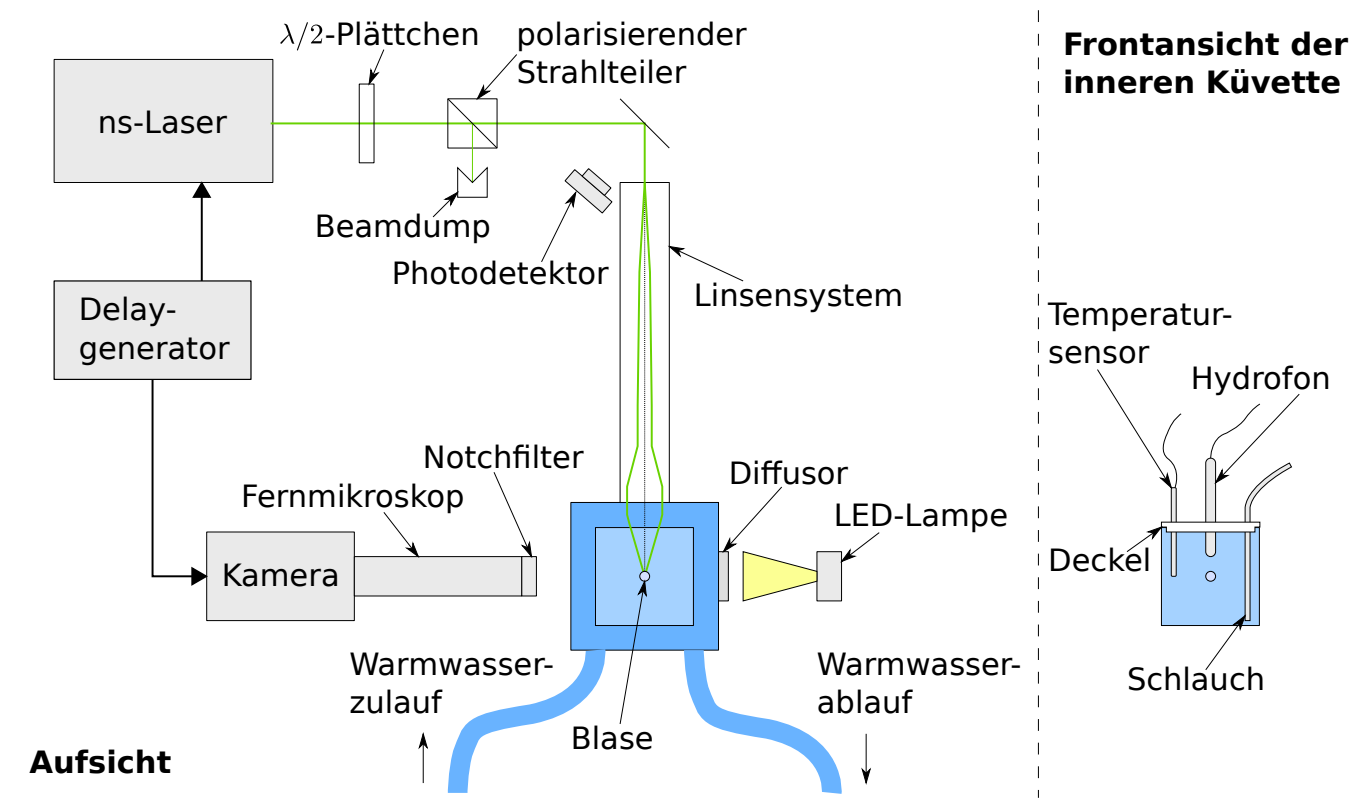

Abbildung 3.1: Experimenteller Aufbau zur Untersuchung von lasererzeugten Blasen bei verschiedenen Wassertemperaturen.

Vor dem Einfüllen in die Küvette wird das deionisierte Wasser mit einer Schlauchpumpe durch einen Membranfilter (Porengröße: $0.8 \mu \mathrm{m}$ ) geleitet um evtl. noch vorhandene Verunreinigungen zu entfernen.

Bei jeder Messung, d. h. für jede mit dem Laser erzeugte Blase, wird die Wassertemperatur abgelesen. Die einzelnen Messungen wurden in einem Abstand von etwa 2 Minuten gemacht. Beim Wechsel auf die nächsthöhere Temperatur dauert es etwa 30 Minuten, bis sich in der Küvette die gewünschte Temperatur eingestellt hat und diese stabil bleibt. Die Wassertemperatur wird im Bereich von $10^{\circ} \mathrm{C}$ bis $80^{\circ} \mathrm{C}$ stufenweise um $10^{\circ} \mathrm{C}$ erhöht. Die Abweichung der Temperatur vom Mittelwert ist für jede Temperaturstufe kleiner als $0.4^{\circ} \mathrm{C}$.

Eine Hochgeschwindigkeitskamera ermöglicht die Aufnahme der Blasenschwingungen mit bis zu 1 Million Bilder pro Sekunde, so dass die Dynamik einer einzigen Blase in Schritten von $1 \mu$ s abgetastet werden kann. Allerdings muss dazu die Auflösung stark reduziert werden. Der interne Speicher der Hochgeschwindigkeitskamera reicht aus, um die gesamte Dynamik einer einzelnen Blase zu erfassen (hier über einen Zeitraum 
von etwa $300 \mu s)$.

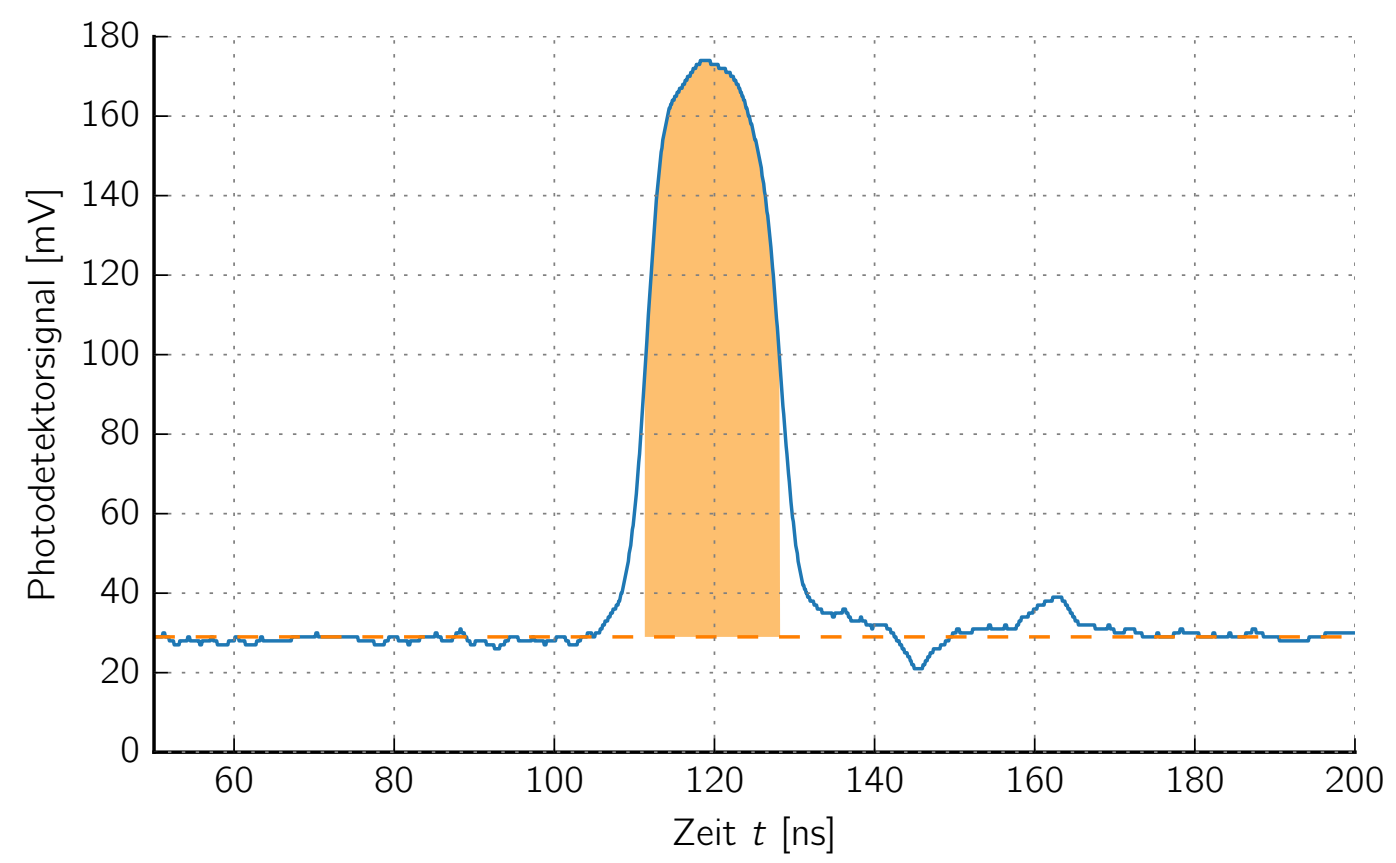

Abbildung 3.2: Ein typisches Photodetektorsignal; aufgenommen während einer Messung bei $T=60.4^{\circ} \mathrm{C}$ Wassertemperatur (Q-Switch Trigger bei $t=0$ ). Der Median ist als gestrichelte Linie eingezeichnet; die orange Fläche dient zur Abschätzung der Pulsenergie.

Die Blasen werden zur Zeit $t=t_{0}$ mit einem Laser (Spectra Physics Quanta Ray PIV400, $\lambda=532 \mathrm{~nm}$, Pulsdauer: $8 \mathrm{~ns}$ ) erzeugt. Bei der hier verwendeten Küvette befindet sich die letzte Linse der Fokussierungsoptik direkt in der Wand der äußeren Küvette. Ein Teil des Laserlichts wird zur Überwachung der Pulsenergie auf einen Photodetektor gelenkt. Direkt nach jeder Messung wird aus dem Photodetektorsignal (Abb. 3.2) die Laserpulsenergie berechnet (Integral über die 1/e-Pulsdauer). Falls diese um mehr als $10 \%$ von einem vorher gewählten Wert abweicht, wird die Messung verworfen. In Abb. 3.3 sind die integrierten Photodetektorsignale für eine gesamte Messreihe eingetragen. Der Mittelwert ist als gestrichelte Linie eingezeichnet; die Standardabweichung beträgt $5 \%$.

Die Hochgeschwindigkeitsaufnahmen werden mit der Kamera Photron SA5 und dem Fernmikroskop Infinity K2 mit CF-3 Objektiv aufgenommen. Vorne am Objektiv ist ein Notchfilter montiert, der das Laserlicht blockiert. Ein Nadelhydrofon mit nachgeschalte- 


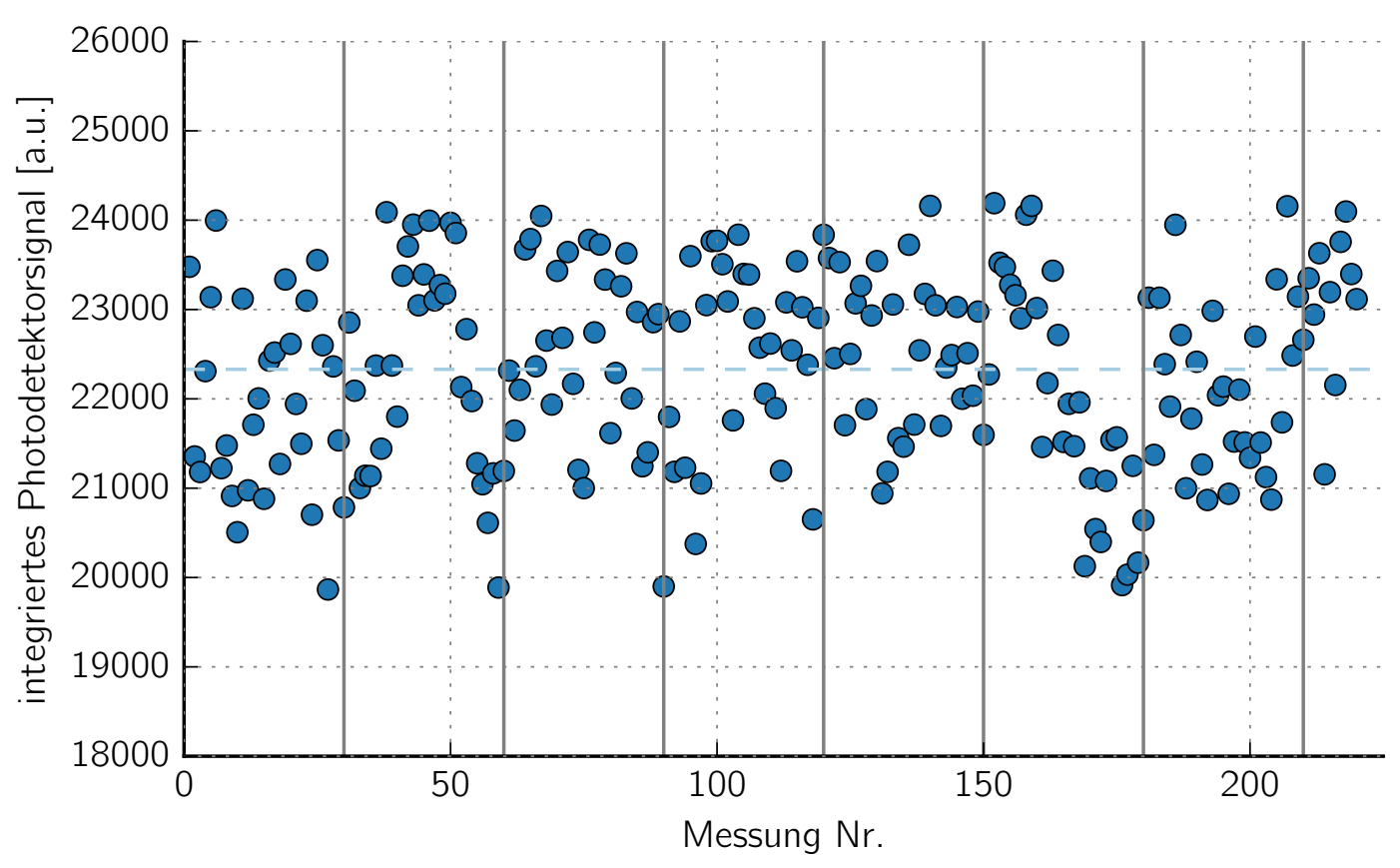

Abbildung 3.3: Integrierte Photodetektorsignale für eine gesamte Messreihe. Die durchgezogenen vertikalen Linien zeigen einen Wechsel der Wassertemperatur an. Der Mittelwert ist als gestrichelte Linie eingezeichnet. Die Standardabweichung beträgt $5 \%$.

tem Verstärker misst die von der Blase ausgehenden Stoßwellen. Für jede lasererzeugte Blase werden beide Signale (Hydrofon und Photodetektor) jeweils auf einem Oszilloskop (Tektronix TDS 2014 bzw. Tektronix TDS 224, beide $100 \mathrm{MHz}$ Bandbreite) digitalisiert und anschließend von einem PC ausgelesen. Das Hydrofonsignal wird zusätzlich mit einem schnelleren Oszilloskop (HP Infinium, 1.5 GHz Bandbreite, 4GS/s Abtastrate) aufgezeichnet, um die kurzen Schalldruckpulse der Stoßwellen besser auflösen zu können. Als Trigger für die Oszilloskope wird das Q-Switch-Triggersignal des Lasers verwendet. Während der Aufnahme wird die Blase von hinten kontinuierlich mit einer LED-Lampe beleuchtet; eine Mattscheibe sorgt für diffuses Licht.

Die Bildwiederholrate der Kamera wird auf 300000 fps eingestellt, so dass der Bildabstand $3.33 \mu$ s beträgt. Die Belichtungszeit wird auf $1 \mu$ s festgelegt. Der Bildausschnitt ist damit auf $256 \times 64$ Pixel begrenzt. Diese Einstellung stellt einen optimalen Kompromiss zwischen Auflösung und Bildwiederholrate dar. Die Vergrößerung des Fernmikroskops ist so gewählt, dass die Blasen im maximal aufgeschwungenen Zustand die verfügba- 


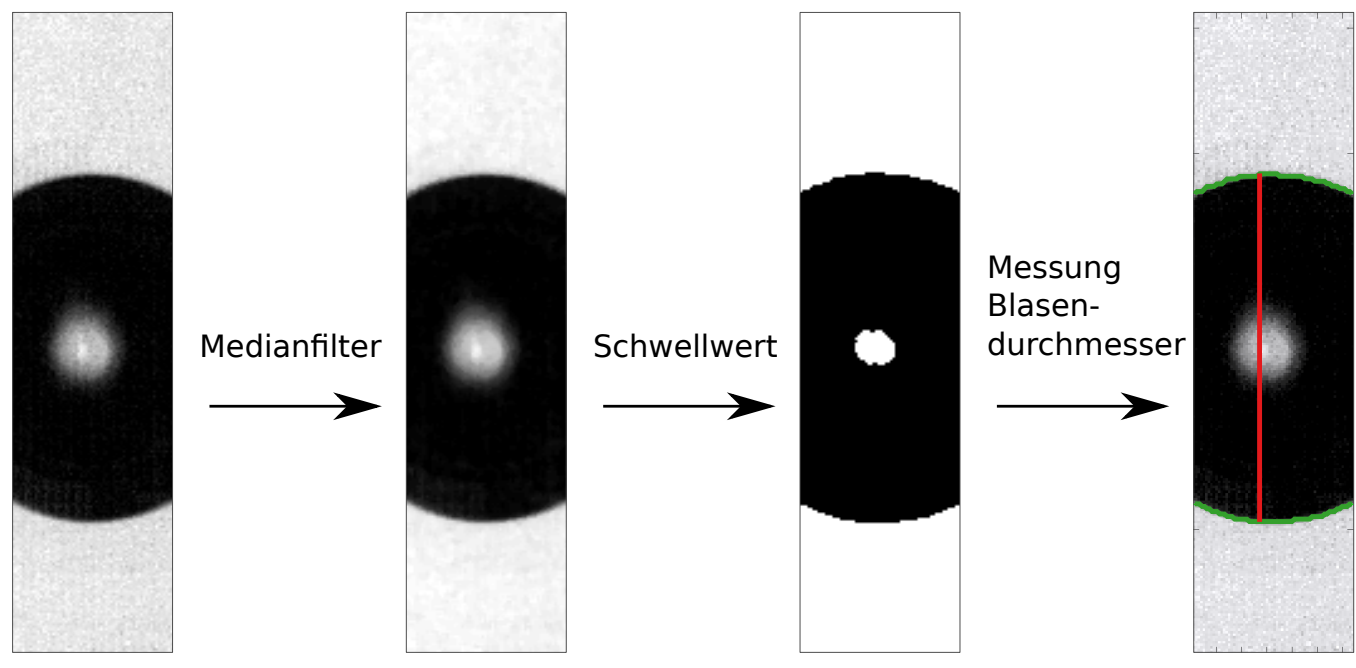

Rohdaten

Abbildung 3.4: Bestimmung des Blasenradius aus den Hochgeschwindigkeitsaufnahmen. Die grüne Linie markiert den Blasenrand, die rote zeigt den gefundenen Blasendurchmesser.

re Breite von 256 Pixeln möglichst gut ausfüllen. Da die Höhe des Bildausschnitts nur 64 Pixel beträgt, wird in dieser Richtung ein Teil der Blase abgeschnitten. Der Blasenradius lässt sich aber in der anderen Richtung bestimmen (siehe Abb. 3.4.

\subsection{Numerische Simulationen}

Für die numerischen Rechnungen werden hier drei verschiedene Modelle für sphärische Blasen herangezogen, die in Abschnitt 2.1 beschrieben werden: Gilmore [13], Eick [20] und Toegel [24]. Alle drei Modelle basieren auf gewöhnlichen Differentialgleichungen, die mit einem Runge-Kutta Verfahren integriert werden. Dabei kommt der Löser dopri 5 aus der Klasse scipy. integrate. ode der Bibliothek SciPy [60] zum Einsatz.

Die verwendeten Modelle erfassen nicht die Erzeugung und das Aufschwingen einer lasererzeugten Blase. Die Simulation wird daher im maximalen Blasenradius gestartet. 
Für die Rechnungen werden der Blasenradius $R(0)$ und die Blasenwandgeschwindigkeit $\dot{R}(0)$ als Anfangswerte benötigt. Als Parameter treten in allen Modellen der Ruheradius $R_{\mathrm{n}}$, die Viskosität $\mu$ der Flüssigkeit, die Oberflächenspannung $\sigma$, der Dampfdruck $p_{\mathrm{v}}(T)$ und die Wassertemperatur $T$ auf.

Der Ruheradius ist schwierig zu bestimmen, da sich nur mit hohem experimentellen Aufwand feststellen ließe, was sich im Inneren einer Laserblase befindet (Stoffzusammensetzung und -menge). Auch eine theoretische Bestimmung des Blaseninhalts ist schwierig. Lindau [61] gibt einige Abschätzungen für die Wasserdampfmasse im Blaseninneren an. Üblicherweise wird angenommen, dass sich ein kleiner Anteil Gas in einer lasererzeugten Blase befindet. Das Gas kann sich beim Laserdurchbruch durch Plasma-Rekombinationen und chemische Reaktionen bilden oder durch Diffusion in die Blase gelangen.

\subsubsection{Gilmore Modell}

Das Gilmore Modell wurde bereits in Abschnitt 2.1]vorgestellt. Für die numerischen Berechnungen wird am Anfang der Dampfdruck $p_{\mathrm{v}}(T)$ entsprechend der Wassertemperatur festgelegt und bleibt dann für die gesamte Rechnung gleich. Die Flüssigkeit wird als schwach kompressibel betrachtet und als Zustandsgleichung wird die Tait Gleichung verwendet. Das Gas in der Blase wird durch ein Van-der-Waals Gesetz beschrieben. Außerdem werden die Oberflächenspannung sowie die Viskosität der Flüssigkeit berücksichtigt; diese Größen sind aber nicht temperaturabhängig.

\subsubsection{Eick Modell}

Gegenüber dem Gilmore Modell gibt es im Eick Modell [20] eine zusätzliche gewöhnliche Differentialgleichung für den Gasdruck $p_{\mathrm{g}}$ in der Blase (siehe Abschnitt 2.1). Damit lässt sich die Wärmeleitung über die Blasenwand beschreiben.

In diesem Modell ist zusätzlich ein Anfangswert für den Gasdruck $p_{\mathrm{g}}(0)$ in der Blase nötig. Dieser wird abhängig vom Anfangsradius $R(0)$ auf

$$
p_{\mathrm{g}}(0)=\left(p_{\mathrm{stat}}+\frac{2 \sigma}{R_{\mathrm{n}}}-p_{\mathrm{v}}\right)\left(\frac{R_{\mathrm{n}}^{3}-b R_{\mathrm{n}}^{3}}{R(0)^{3}-b R_{\mathrm{n}}^{3}}\right)^{\kappa}
$$

gesetzt. Der Dampfdruck $p_{\mathrm{v}}$ wird am Anfang aus der Temperatur der Flüssigkeit bestimmt und bleibt dann für die gesamte Rechnung fest. Die Viskosität der Flüssigkeit, 
die Oberflächenspannung und die Schallgeschwindigkeit in der Flüssigkeit sind in diesem Modell nicht temperaturabhängig.

\subsubsection{Toegel Modell}

Das Toegel Modell wird in Abschnitt2.1 beschrieben. Da es sich in dem hier vorgestellten Experiment um eine freie Blasenschwingung handelt, gibt es im Gegensatz zu [24] keine externe Schallanregung $\left(p_{\text {ext }}=0\right)$. Als Anfangswerte werden $R(0), \dot{R}(0), N(0)$ und $T(0)=T_{1}$ vorgegeben. Die Parameter des Modells sind die Temperatur der Flüssigkeit $T_{1}$ und der Gleichgewichtsradius $R_{\mathrm{n}, \mathrm{g}}$ für eine reine Gasblase ohne Wasserdampf (also bei $N=0$ ). Außerdem gibt es noch einige Stoffparameter, die von der Flüssigkeit und vom Gas in der Blase abhängig sind. Als Flüssigkeit wird hier Wasser angenommen und innerhalb der Blase befindet sich vor allem Wasserdampf. Es kann auch ein kleiner Gasanteil berücksichtigt werden (siehe Abschnitt 2.1). Bei lasererzeugten Blasen ist, wie weiter oben schon erwähnt, nicht klar, welche Gassorten und welche Gasmenge sich in der Blase befinden. Hier wird angenommen, dass die Blase Wasserstoff enthält.

Da die Rechnung beim Maximalradius gestartet wird und sich der Blasenradius zunächst kaum ändert, kann man davon ausgehen, dass sich in der Blase der Dampfdruck einstellt. Der Anfangswert für $N$ wird mit Hilfe der idealen Gasgleichung aus dem Dampfdruck bestimmt:

$$
N(0)=\frac{p_{\mathrm{v}}(T(0))}{R_{\mathrm{g}} T(0)} \frac{4 \pi}{3} R(0)^{3}
$$

Abbildung 3.5 zeigt die Ergebnisse des Toegel Modells bei Raumtemperatur. Die Ergebnisse für eine höhere Temperatur $\left(T_{1}=40^{\circ} \mathrm{C}\right)$ sind in Abb. 3.6 dargestellt. Der Ruheradius $R_{\mathrm{n}}$ der Blase nimmt nach jedem Kollaps ab, da Wasserdampf an der Blasenwand kondensiert und dadurch die Stoffmenge $N$ abnimmt.

\subsubsection{Vergleich der verschiedenen Blasenmodelle}

In Abb. 3.7 sind zum Vergleich der drei Modelle die Ergebnisse der Rechnungen bei Raumtemperatur $\left(T=21^{\circ} \mathrm{C}\right)$ und bei einer höheren Wassertemperatur $\left(T=40^{\circ} \mathrm{C}\right)$ dargestellt. Für alle Rechnungen wurden mit Ausnahme des Ruheradius $R_{\mathrm{n}}$ bzw. $R_{\mathrm{n}, \mathrm{g}}$ die gleichen Parameter und Anfangsbedingungen verwendet: Anfangsradius $R(0)=$ $350 \mu \mathrm{m}$, Blasenwandgeschwindigkeit $\dot{R}(0)=10^{-9} \mathrm{~m} \mathrm{~s}^{-1}$. Der Ruheradius wird jeweils 

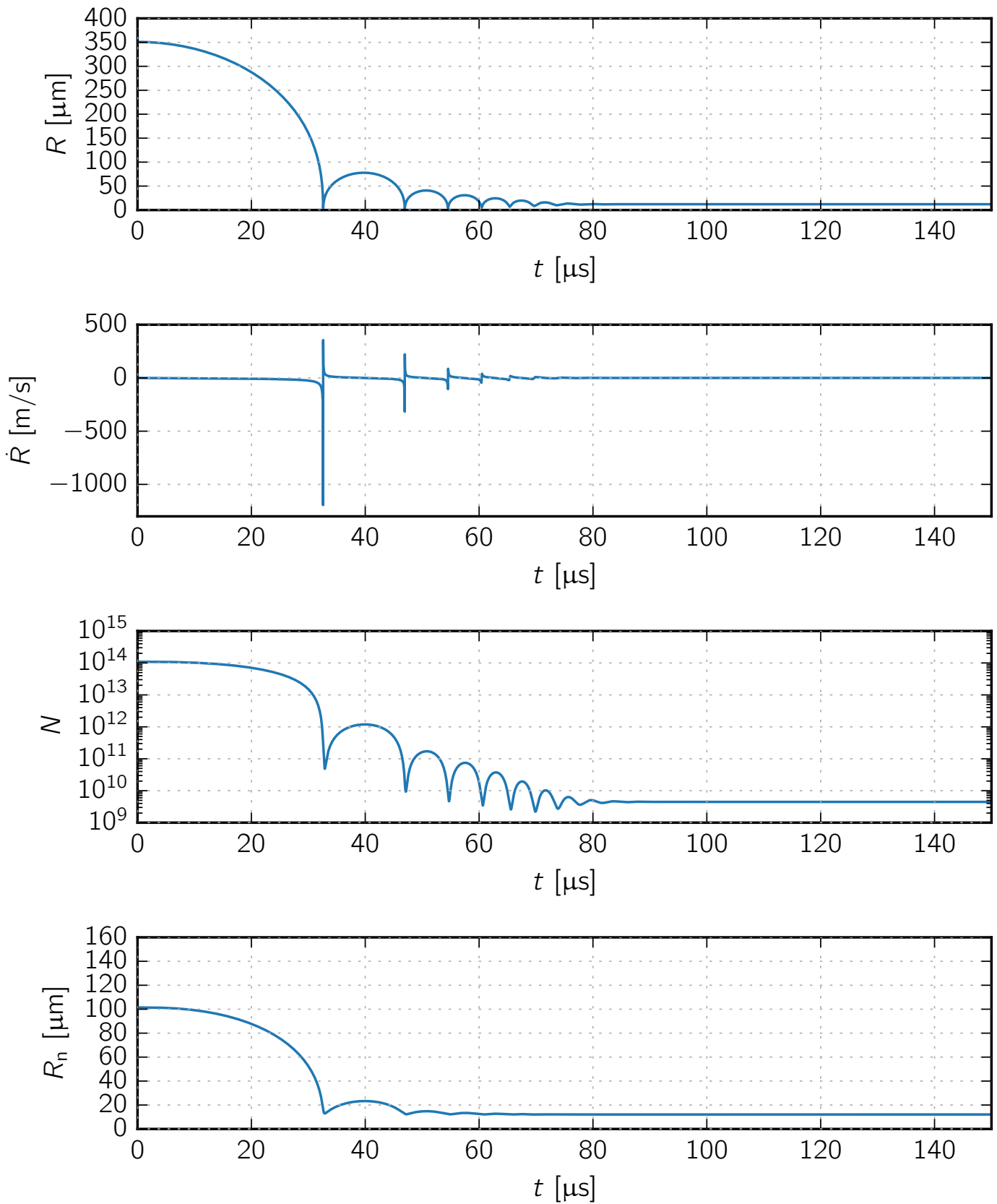

Abbildung 3.5: Ergebnisse der numerischen Rechnungen nach dem Toegel Modell bei einer Wassertemperatur von $T_{1}=21^{\circ} \mathrm{C}$ und einem Ruheradius von $R_{\mathrm{n}, \mathrm{g}}=12 \mu \mathrm{m}$. Dargestellt sind der Blasenradius $R$, die Blasenwandgeschwindigkeit $\dot{R}$, die Stoffmenge $N$ für den Wasserdampf in der Blase und der Ruheradius $R_{\mathrm{n}}$, der über die Gl. (2.32) mit $N$ zusammenhängt. 

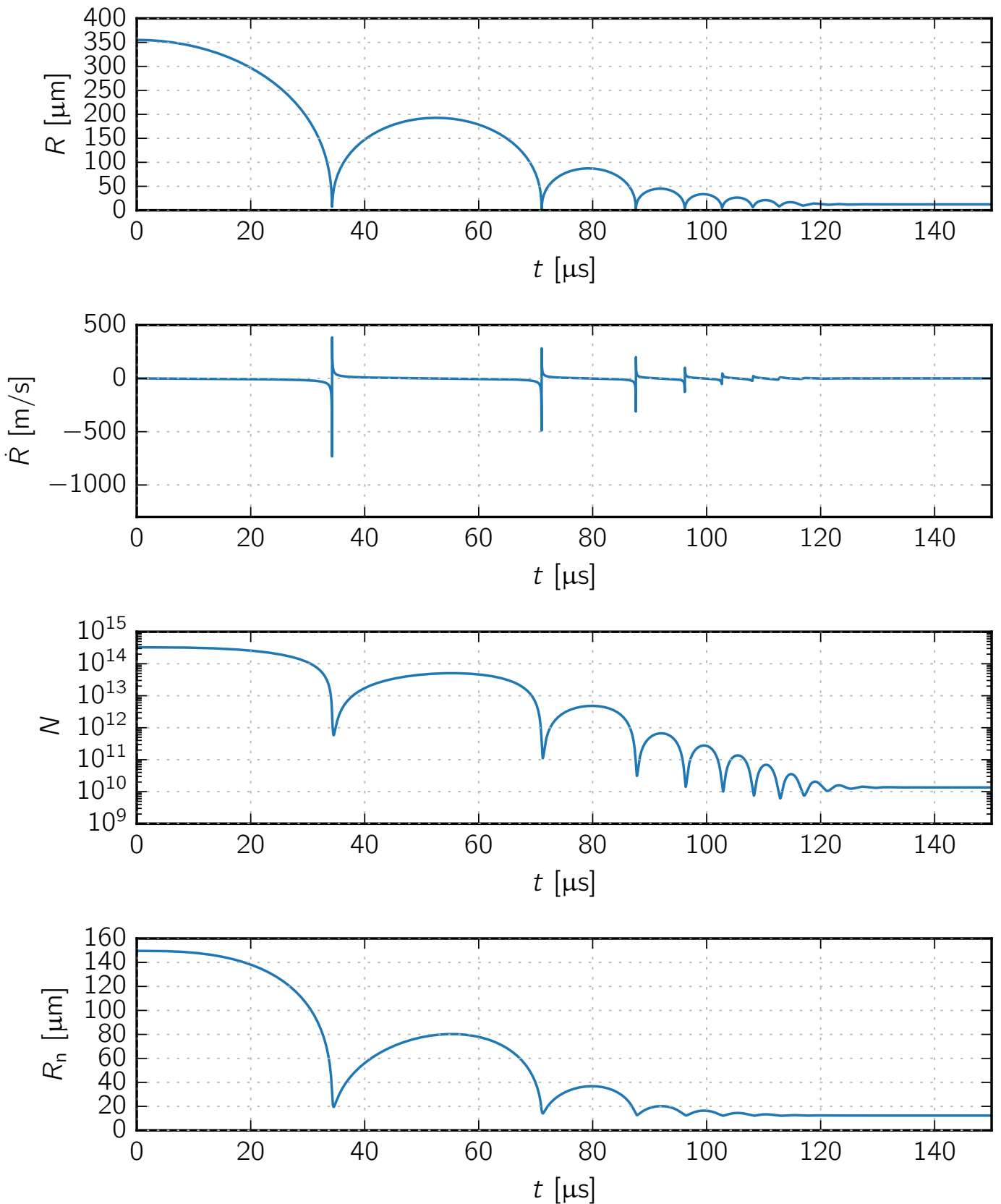

Abbildung 3.6: Ergebnisse der numerischen Rechnungen nach dem Toegel Modell bei einer Wassertemperatur von $T_{1}=40^{\circ} \mathrm{C}$ und einem Ruheradius von $R_{\mathrm{n}, \mathrm{g}}=12 \mu \mathrm{m}$. Dargestellt sind der Blasenradius $R$, die Blasenwandgeschwindigkeit $\dot{R}$, die Stoffmenge $N$ für den Wasserdampf in der Blase und der Ruheradius $R_{\mathrm{n}}$, der über die Gl. 2.32) mit $N$ zusammenhängt. 
so gewählt, dass die Modelle bis zum 2. Kollaps den gleichen Blasenradius liefern. Für das Eick Modell wurde am Anfang der Gasdruck in der Blase aus dem Anfangsradius $R(0) \mathrm{zu} p_{\mathrm{g}}(0)=10 \mathrm{~Pa}$ bestimmt (siehe oben). Im Toegel Modell ergibt sich bei $T(0)=$ $21^{\circ} \mathrm{C}$ für die Stoffmenge ein Anfangswerte von $N(0)=1.10 \cdot 10^{14}$; bei $T(0)=40^{\circ} \mathrm{C}$ erhält man $N(0)=3.07 \cdot 10^{14}$.

Im Eick Modell ist im zweiten Rebound der Blasenradius kleiner als im Gilmore Modell, da die Blasenschwingung durch die zusätzlich vorhandene Wärmeleitung stärker gedämpft wird. Im Toegel Modell kann auch Wasserdampf kondensieren, wodurch die Blasenschwingung noch weiter gedämpft wird.

Eine Änderung der Wassertemperatur von $21^{\circ} \mathrm{C}$ auf $40^{\circ} \mathrm{C}$ hat sowohl im Gilmore als auch im Eick Modell kaum einen Einfluss auf den maximalen Blasenradius im ersten Rebound. Im Gilmore Modell steigt er von $76 \mu \mathrm{m}$ auf $77 \mu \mathrm{m}$; im Eick Modell bleibt er bei $76 \mu \mathrm{m}$. Im Toegel Modell dagegen steigt bei $T_{1}=40^{\circ} \mathrm{C}$ der Blasenradius im ersten Rebound auf $193 \mu \mathrm{m}$ im Vergleich zu $78 \mu \mathrm{m}$ bei $T_{1}=21^{\circ} \mathrm{C}$.

Beim Wechsel auf die höhere Wassertemperatur $\left(T=40^{\circ} \mathrm{C}\right)$ verlängert sich im Fall von Gilmore und Eick die Kollapszeit um $1.3 \mu \mathrm{s}$, im Toegel Modell um 1.7 $\mu \mathrm{s}$. Die Blase kollabiert bei $40^{\circ} \mathrm{C}$ also langsamer.

Das Toegel Modell beschreibt das Verhältnis der Maximalradien in aufeinanderfolgenden Rebounds besser als die anderen beiden Modelle (siehe Abschnitt 3.3.2).

\subsection{Ergebnisse}

Im Folgenden werden die Ergebnisse der Hochgeschwindigkeitsaufnahmen ausgewertet und diskutiert. Anschließend werden die Messdaten mit den Ergebnissen der numerischen Rechnungen verglichen.

\subsubsection{Hochgeschwindigkeitsaufnahmen}

Die mit der Hochgeschwindigkeitskamera aufgenommenen Videos liegen als AVI-Datei vor. Mit einem Matlabprogramm werden zunächst die Einzelbilder extrahiert, um sie anschließend weiter zu verarbeiten. Als Beispiel ist in Abb. 3.8 eine Bildsequenz dargestellt, die bei einer Wassertemperatur von $T=20.7^{\circ} \mathrm{C}$ aufgenommen wurde. Für 

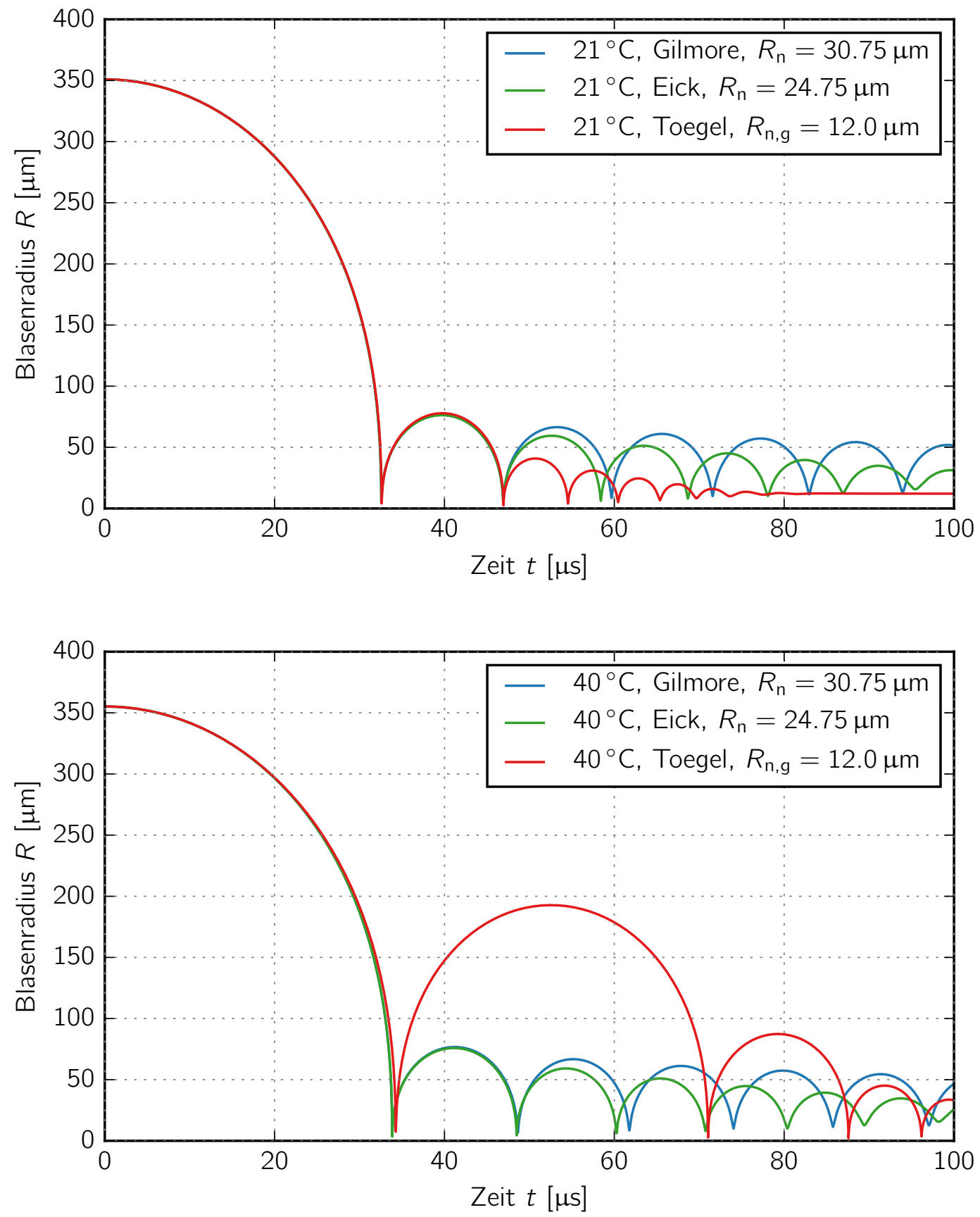

Abbildung 3.7: Vergleich von Gilmore, Eick und Toegel Modell bei einer Wassertemperatur von $21^{\circ} \mathrm{C}$ (oben) und $40^{\circ} \mathrm{C}$ (unten). Anfangswerte: $R(0)=350 \mu \mathrm{m}$, $\dot{R}(0)=10^{-9} \mathrm{~m} \mathrm{~s}^{-1}$. 


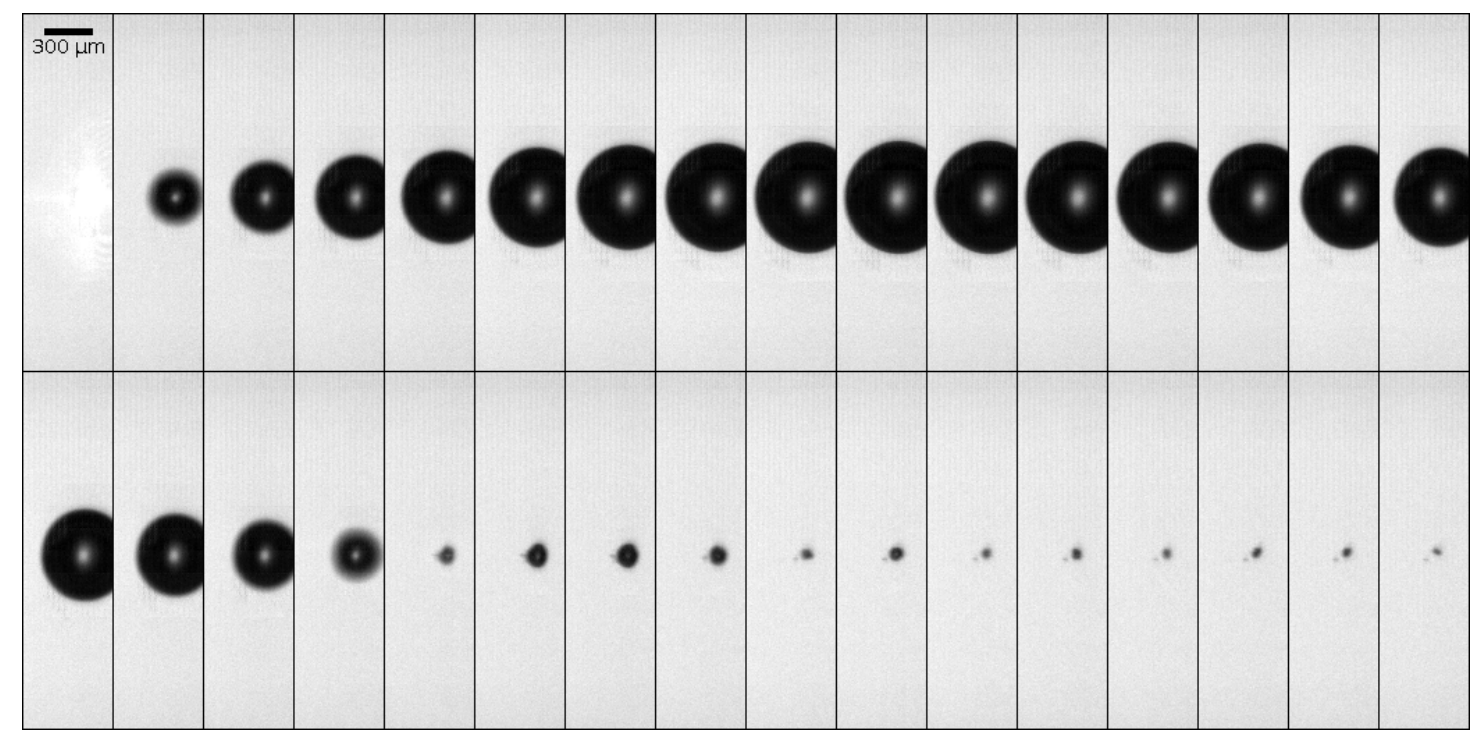

Abbildung 3.8: Bildsequenz einer lasererzeugten Blase bei $T=20.7^{\circ} \mathrm{C}$, aufgenommen mit der Hochgeschwindigkeitskamera. Bildabstand: $3.3 \mu \mathrm{s}$.

jedes einzelne Bild soll nun der Blasenradius bestimmt werden. Der Programmablauf dazu ist in Abb. 3.4 an einem Beispielbild veranschaulicht. Im ersten Schritt wird zur Reduzierung des Rauschens ein Medianfilter angewendet. Dann wird anhand eines Schwellwertes entschieden, welche Pixel zur Blase gehören und welche zum Hintergrund. Schließlich kann der Blasenradius bestimmt werden. Dies geschieht wegen des länglichen Bildausschnitts nur in Richtung der längeren Bildachse. Für jede Spalte des Bildes wird von oben und von unten aus der Blasenrand gesucht. Damit erhält man pro Spalte zwei Punkte auf dem Blasenrand, deren Abstand abgespeichert wird. Der Maximalwert all dieser Abstände ist der Blasendurchmesser. Falls die Blase sich in mehrere kleinere Blasen aufspaltet, wird dies vom Algorithmus erkannt. In diesem Fall kann kein Blasenradius angegeben werden.

Im Experiment (siehe Abb. 3.1) ist die Kamera um $90^{\circ}$ um die optische Achse des Fernmikroskops gedreht, so dass der Laserpuls im Originalvideo von unten kommt. Die einzelnen Bilder der Abb. 3.8 wurden so gedreht, dass hier der Laserpuls von rechts kommt. Die Kamera wurde für die dargestellte Sequenz zeitgleich mit dem Q-Switch des Lasers getriggert. Da das Triggersignal zunächst die Kabel und die Elektronik im Steuergerät des Lasers durchlaufen muss, ist der Laserpuls um einige Nanosekunden verzögert (siehe Abb. 3.2. Im ersten Bild der Sequenz in Abb. 3.8 hat sich bereits ein Plasma gebildet (im Bild weiß und daher kaum zu erkennen). Danach wächst die Blase bis zu einem gewissen Maximalradius an und kollabiert anschließend. Nach dem 
ersten Kollaps schwingt sie nochmal auf, bleibt aber sehr viel kleiner als beim ersten Aufschwingen.

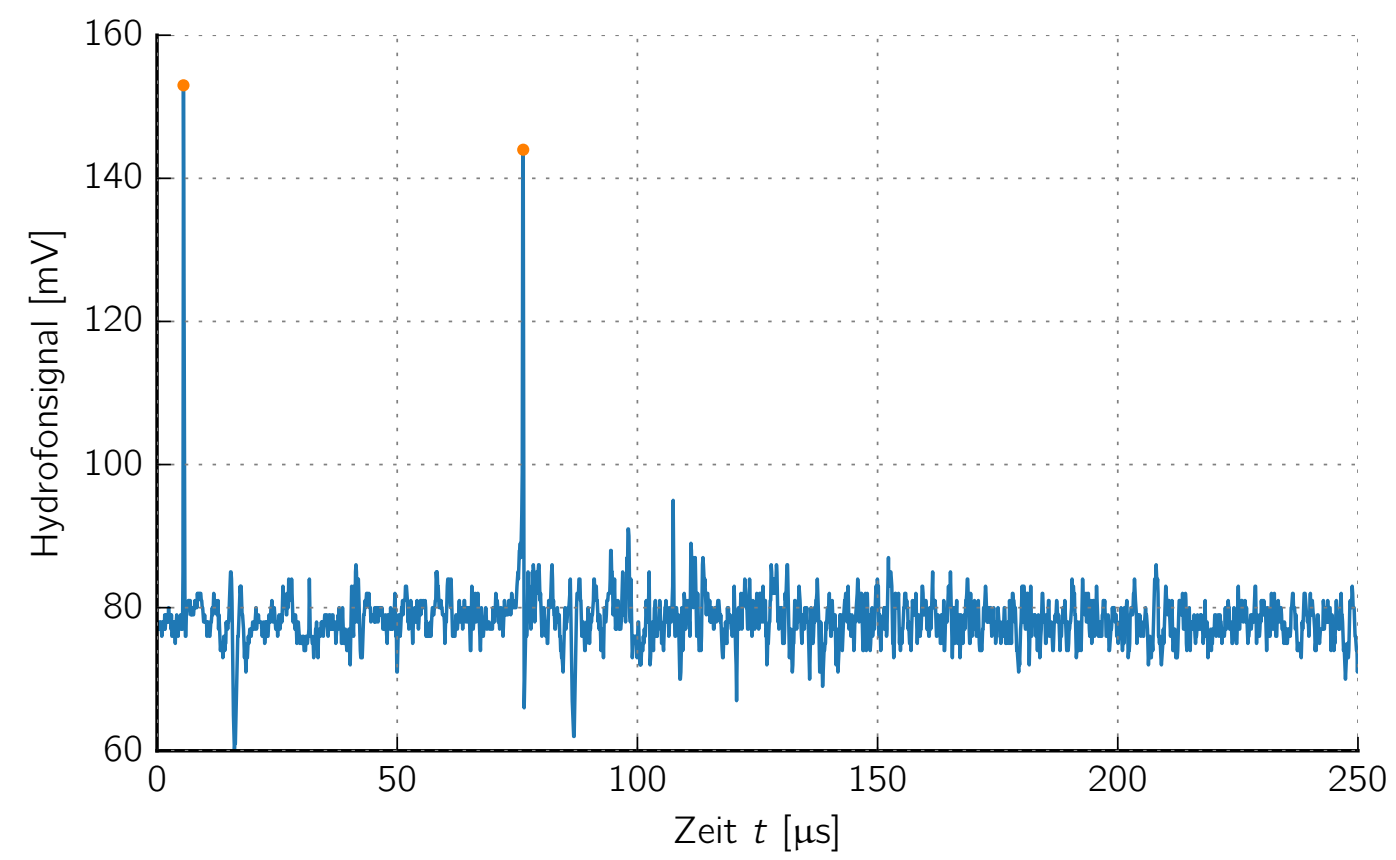

Abbildung 3.9: Typischer Verlauf einer Hydrofonaufzeichnung bei einer Wassertemperatur von $T=60.4^{\circ} \mathrm{C}$. Die Stoßwellen, die bei der Blasenerzeugung und beim ersten Kollaps entstehen, sind als Peak erkennbar. Der zeitliche Abstand der beiden Stoßwellen beträgt $71 \mu$ s.

Bei der Erzeugung einer Blase mit dem Laser wird eine Stoßwelle ausgesandt, die im Hydrofonsignal als Peak erkennbar ist. Bei jedem Kollaps geht eine weitere Stoßwelle von der Blase aus. In Abb. 3.9 sind die Stoßwellen bei der Blasenerzeugung und beim ersten Kollaps zu sehen. Der zeitliche Abstand der beiden Peaks im Hydrofonsignal entspricht der doppelten Kollapszeit, also $2 t_{c}$, und beträgt hier $71 \mu \mathrm{s}$.

Aus allen Hochgeschwindigkeitsaufnahmen wurde der Blasenradius ermittelt und gegen die Zeit aufgetragen. Die erstellten Radius-Zeit-Kurven für einige ausgewählte Wassertemperaturen sind in Abb. 3.10 dargestellt. Alle aufgenommenen Messungen werden nach dem maximalen Blasenradius sortiert. Für die gezeigten Messungen beträgt der maximale Blasenradius $R_{\max } \approx 350 \mu \mathrm{m}$. Im ersten Rebound ist der Einfluss der Wassertemperatur auf die Blasendynamik deutlich zu erkennen: Bei hohen Temperaturen ist in dieser Phase der maximale Blasenradius wesentlich größer als bei niedrigen. Insbe- 


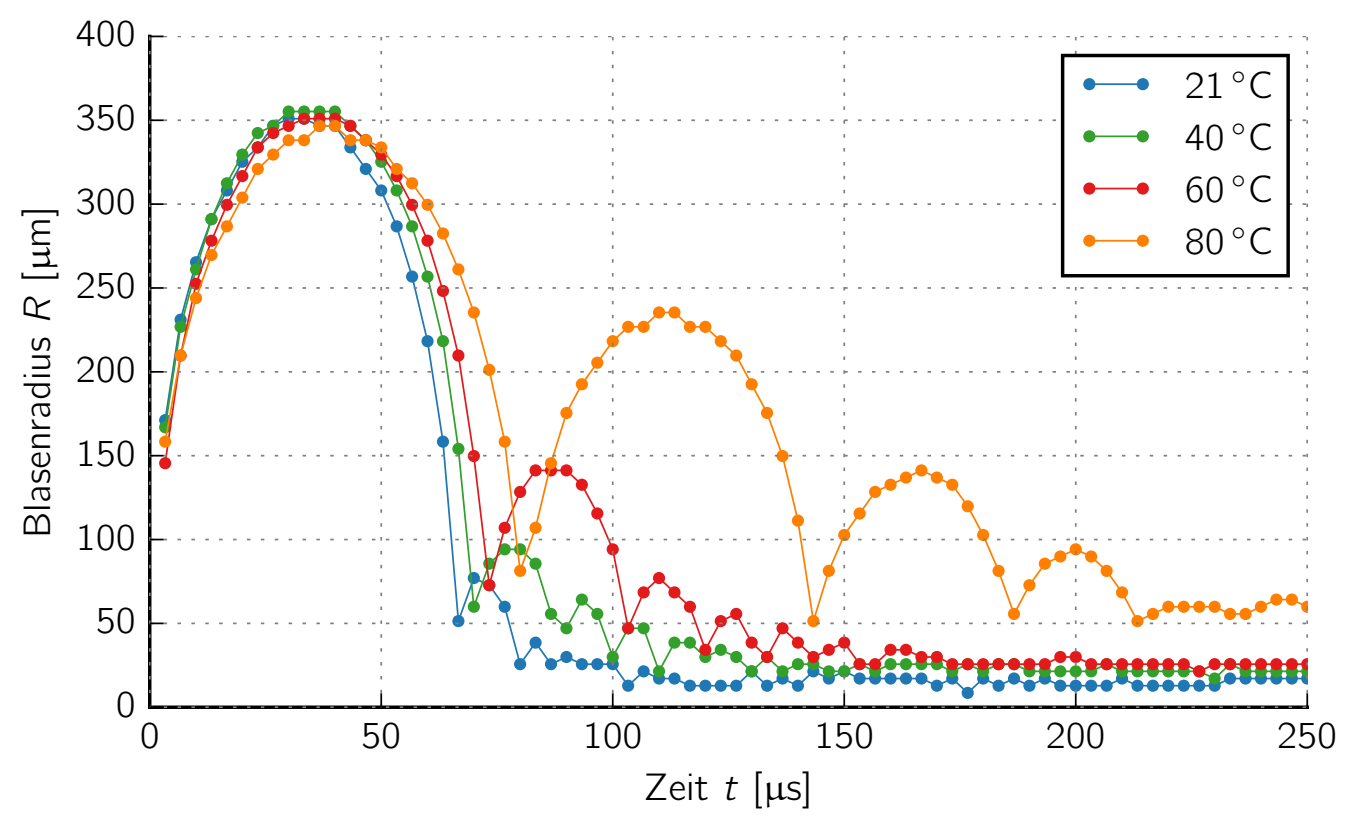

Abbildung 3.10: Blasenradius als Funktion der Zeit bei verschiedenen Wassertemperaturen für ausgewählte Blasen mit $R_{\max } \approx 350 \mu \mathrm{m}$.

sondere bei der höchsten hier dargestellten Temperatur von $T=80^{\circ} \mathrm{C}$ ist dies auch in den späteren Rebounds zu sehen.

Zum Vergleich sind in Tab. 3.1 die maximalen Blasenradien im ersten Rebound für die in Abb. 3.10 gezeigten Messungen angegeben. Bei $T=80^{\circ} \mathrm{C}$ ist der maximale Radius im ersten Rebound mehr als dreimal so groß wie bei $T=21^{\circ} \mathrm{C}$. Bei der höheren Temperatur schwingt die Blase im ersten Rebound bis auf zwei Drittel ihres maximalen Radius auf. Bei einer Wassertemperatur von $T=21^{\circ} \mathrm{C}$ erreicht sie im ersten Rebound hingegen nur $22 \%$ ihres maximalen Radius.

Abbildung 3.11 zeigt ergänzend zu Abb. 3.10 die Radius-Zeit-Kurven für zwei weitere ausgewählte Maximalradien $\left(R_{\max } \approx 300 \mu \mathrm{m}\right.$ und $\left.R_{\max } \approx 400 \mu \mathrm{m}\right)$. Bei größerem Maximalradius wird auch der maximale Radius im Rebound größer. Für das Verhältnis von $R_{\max , \mathrm{Rb} 1}$ zu $R_{\max }$ für die Blasen in Abb. 3.11 ergeben sich ähnliche Werte wie bei $R_{\max } \approx 350 \mu \mathrm{m}$ (siehe Tab. 3.1). Beispielsweise erhält man bei $R_{\max } \approx 300 \mu \mathrm{m}$ und $T=80^{\circ} \mathrm{C}: R_{\max , \mathrm{Rb} 1} / R_{\max }=0.68$. 

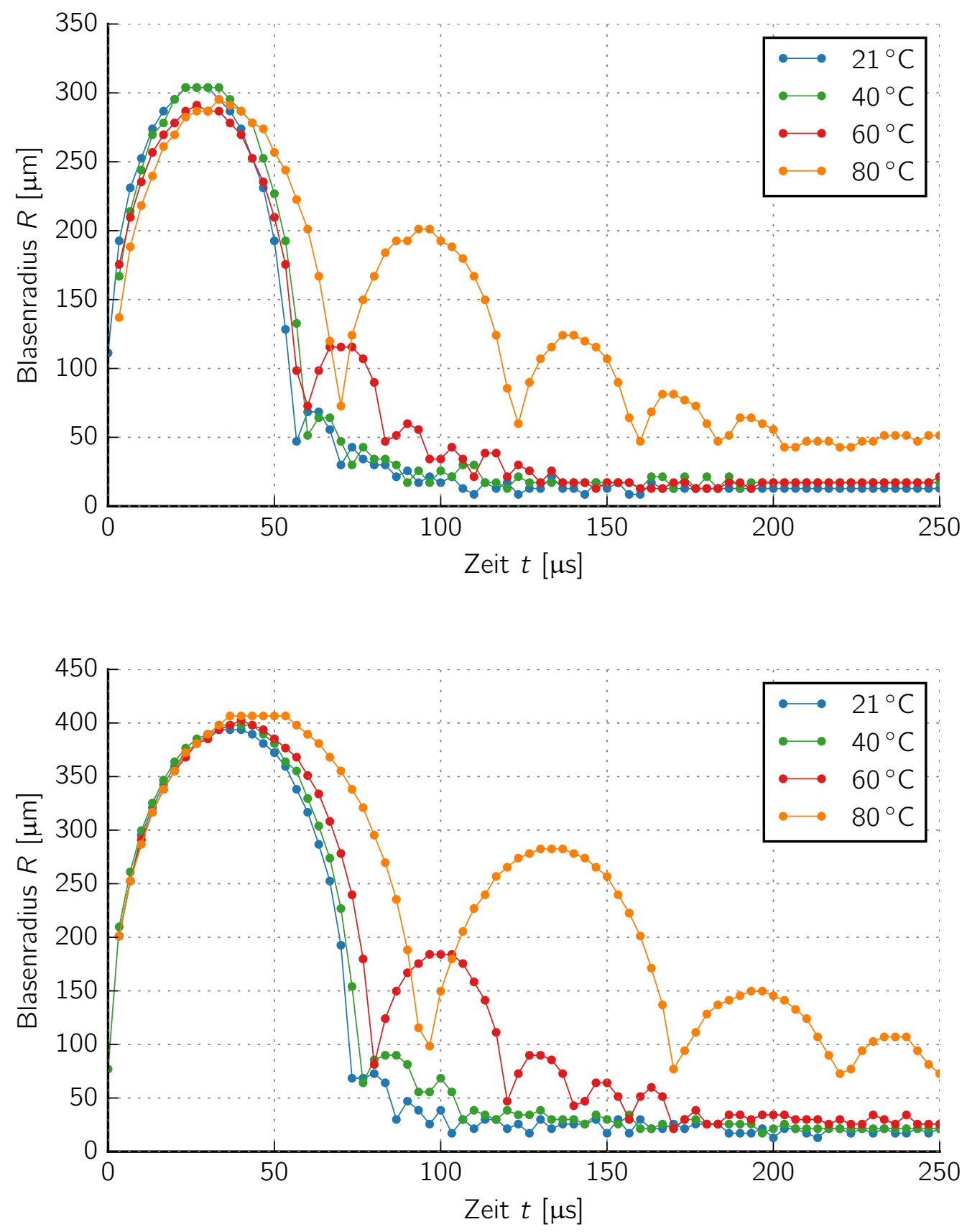

Abbildung 3.11: Blasenradius als Funktion der Zeit bei verschiedenen Wassertemperaturen für ausgewählte Blasen mit $R_{\max } \approx 300 \mu \mathrm{m}$ (oben) und $R_{\max } \approx$ $400 \mu \mathrm{m}$ (unten). 


\begin{tabular}{lccc}
\hline$T\left[{ }^{\circ} \mathrm{C}\right]$ & $R_{\max , \mathrm{Rb} 1}[\mu \mathrm{m}]$ & $R_{\max , \mathrm{Rb} 1} / R_{\max }$ & $R_{\max , \mathrm{Rb} 1} /\left(\left.R_{\max , \mathrm{Rb} 1}\right|_{T=21{ }^{\circ} \mathrm{C}}\right)$ \\
\hline 21 & 77 & 0.22 & 1.0 \\
40 & 94 & 0.27 & 1.2 \\
60 & 141 & 0.40 & 1.8 \\
80 & 235 & 0.67 & 3.1 \\
\hline
\end{tabular}

Tabelle 3.1: Maximaler Blasenradius im 1. Rebound für die Blasen mit $R_{\max } \approx 350 \mu \mathrm{m}$ bei verschiedenen Wassertemperaturen.

Der größere maximale Blasenradius im Rebound bei hohen Wassertemperaturen ist auf den erhöhten Dampfdruck zurückzuführen. Dieser führt dazu, dass die in den Blasen enthaltene Dampfmenge größer ist.

Bei hohen Wassertemperaturen läuft der Kollaps langsamer ab (Abbn. 3.10 und 3.16). Eine Erhöhung der Temperatur von $21^{\circ} \mathrm{C}$ auf $60^{\circ} \mathrm{C}$ führt zu einem Anstieg der Kollapszeit um $2 \mu$ s von $35 \mu$ s auf $37 \mu \mathrm{s}$. Bei einer Temperaturänderung von $40^{\circ} \mathrm{C}$ auf $80^{\circ} \mathrm{C}$ verlängert sich die Kollapszeit um $7 \mu$ s von $35 \mu$ s auf $42 \mu$ s.

Der minimale Blasenradius im Kollaps ist bei hohen Temperaturen größer. Für die in Abb. 3.10 gezeigten Messungen nimmt der minimale Blasenradius im ersten Kollaps von $51 \mu \mathrm{m}$ (bei $T=21^{\circ} \mathrm{C}$ ) bis auf $81 \mu \mathrm{m}$ (bei $T=80^{\circ} \mathrm{C}$ ) zu. Allerdings ist der tatsächlich erreichte Minimalradius sicherlich kleiner, da die zeitliche Auflösung von $3.3 \mu$ s bei diesem Experiment nicht ausreicht, um den schnellen Blasenkollaps zu erfassen.

Da die Blase bei hohen Wassertemperaturen im Kollaps nicht so klein wird (minimaler Blasenradius ist größer), ist der maximale Druck der abgestrahlten Stoßwellen kleiner. Es wird dann weniger Energie in Form von Stoßwellen abgegeben. Die Blase behält mehr Energie für das Aufschwingen im nächsten Rebound und kann einen größeren Maximalradius erreichen.

In Abb. 3.12 sind für zwei ausgewählte Temperaturen die Spitzenwerte im Hydrofonsignal beim 1. Kollaps der Blase für jeweils 30 Messungen dargestellt. Die Spitzenwerte für den Druck der Stoßwellen streuen sehr stark und es ist keine Temperaturabhängigkeit zu erkennen.

Betrachtet man den Mittelwert $\overline{R_{\max }}$ des maximalen Blasenradius für verschiedene Wassertemperaturen bei gleicher Laserpulsenergie (Abb. 3.13), so ist keine eindeutige Abhängigkeit zu erkennen. Um die Signifikanz zu erhöhen, wäre eine größere Anzahl an Messungen nötig. 

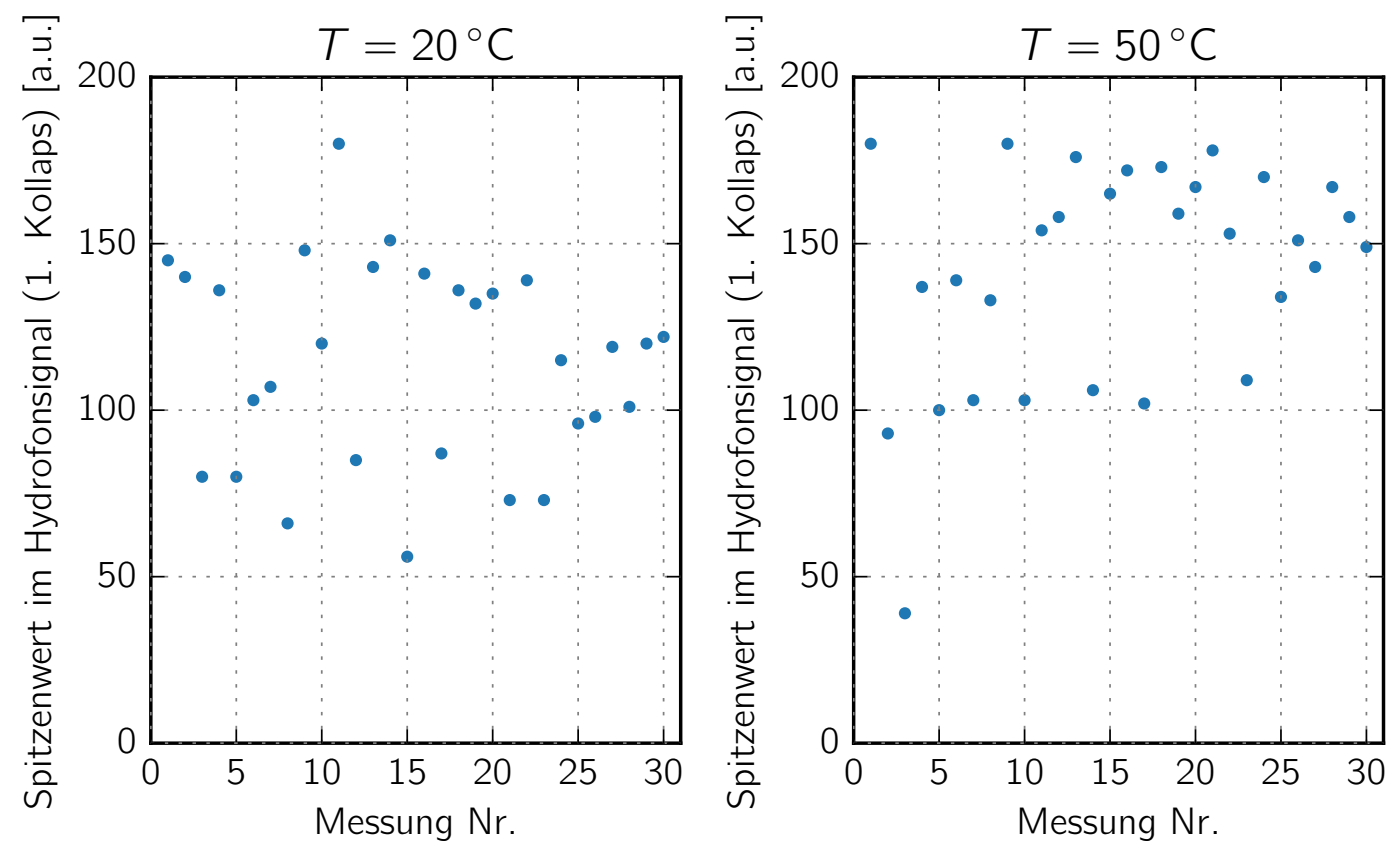

Abbildung 3.12: Spitzenwerte im Hydrofonsignal beim 1. Kollaps für zwei ausgewählte Temperaturen.

Abbildung 3.14 zeigt den maximalen Blasenradius als Funktion der Temperatur für verschiedene Pulsenergien. Der maximale Blasenradius schwankt stark und es ist keine eindeutige Abhängigkeit von der Laserpulsenergie zu erkennen. Eine Ursache für die Schwankungen können Bläschen in der äußeren Temperierküvette sein, die die Fokussierung des Laserpulses beeinflussen.

Der maximale Druck der Stoßwellen hängt auch von der Form der Blase ab. Die stärksten Stoßwellen gibt es bei perfekt kugelförmigen Blasen. Weicht die Blasenform von der Kugelform ab, nimmt der maximale Druck der abgestrahlten Stoßwellen ab. Da im Experiment jede Blase eine etwas andere Form hat, gibt es bei der Messung mit dem Hydrofon eine starke Streuung in den Spitzenwerten des Drucks (siehe Abb. 3.12).

Durch Einstellen der Laserpulsenergie können auch kleinere oder größere Blasen erzeugt werden. Wenn die Laserpulsenergie zu hoch ist, werden die erzeugten Blasen instabil und bleiben nicht mehr kugelförmig (siehe Abb. 3.15). In dieser Bildsequenz hat sich die Blase nach dem ersten Kollaps in zwei kleinere Blasen aufgeteilt. Dies liegt daran, dass der fokussierte Laserpuls eine endliche Ausdehnung hat und das Laserplasma 


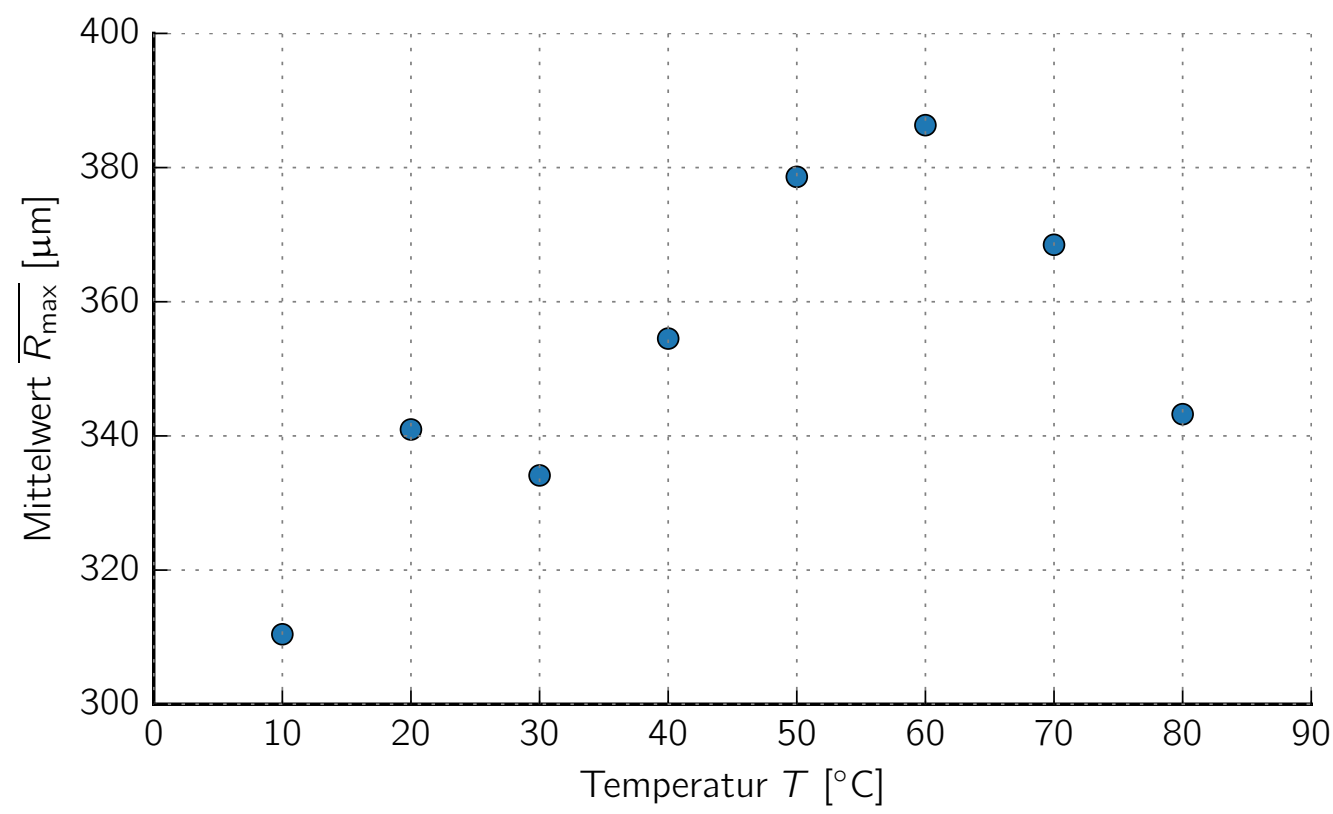

Abbildung 3.13: Mittlerer Maximalradius $\overline{R_{\max }}$ bei verschiedenen Wassertemperaturen. Es wurde jeweils über 30 Messungen gemittelt; bei der höchsten Temperatur nur über 10 Messungen.

etwas in die Länge gezogen ist. Im letzten Bild ist ein zweiter Kollaps zu erkennen.

\subsubsection{Ergebnisse der numerischen Rechnungen und Vergleich mit den Messdaten}

Die numerischen Rechnungen wurden wie in Abschnitt 3.2.2 beschrieben durchgeführt. Der Ruheradius, der als Parameter in die Rechnung eingeht, wurde jeweils durch Anpassung an die Messdaten ermittelt. Bei der Anpassung wird im Zeitfenster vom maximal aufgeschwungenen Zustand $R=R_{\max }$ bis zum 2. Kollaps die Abweichung des Modells von den Messdaten minimiert. Es wird also der Fehler

$$
\sum_{t\left(R_{\max }\right)}^{t(2 . \text { Kollaps })}\left(R_{\text {num }}(t)-R_{\exp }(t)\right)^{2}
$$

minimiert. Die Ergebnisse der numerischen Rechnungen mit dem Eick Modell sind zum Vergleich mit den Messdaten in Abb. 3.16 dargestellt. Abbildung 3.19 zeigt die Ergeb- 


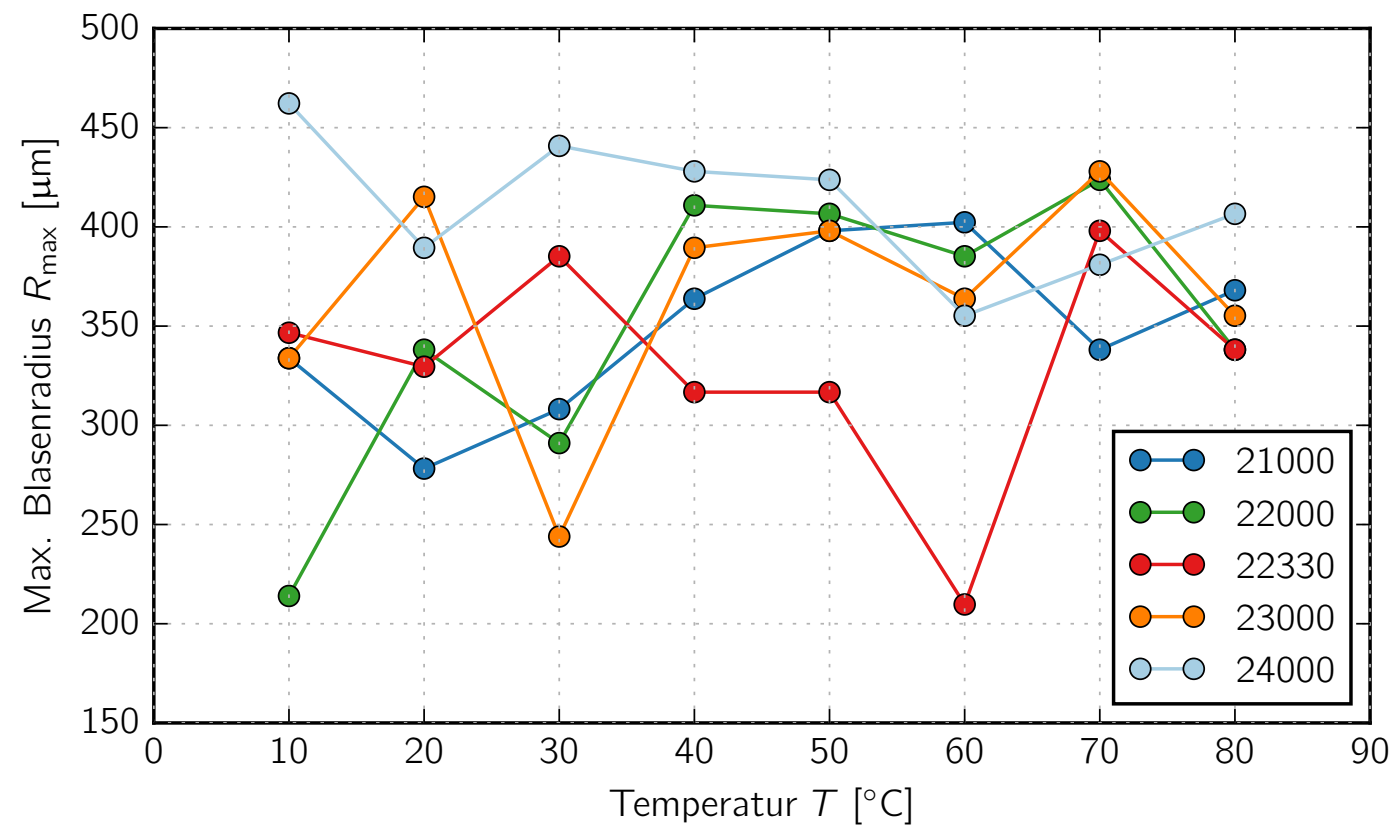

Abbildung 3.14: Maximaler Blasenradius in Abhängigkeit von der Temperatur für verschiedene Pulsenergien.

nisse des Toegel Modells im Vergleich mit den Messdaten.

Modell und Experiment stimmen bis zum 2. Kollaps gut überein. Die längere Kollapszeit bei erhöhten Wassertemperaturen wird durch das Modell richtig beschrieben. Nach dem 2. Kollaps wird der Blasenradius durch das Modell überschätzt und der nächste Kollaps findet später als im Experiment statt.

Die durch Anpassung des Eick Modells an die Messdaten bestimmten Ruheradien für verschiedene Wassertemperaturen sind in Abb. 3.17 eingetragen. Es ist auch eine Anpassung der Funktion $R_{\mathrm{n}}(T)=a T^{3}+b$ an die Ruheradien dargestellt.

Abbildung 3.18 zeigt die gleichen Werte für den Ruheradius wie in Abb. 3.17, mit dem Unterschied, dass hier die Abszisse den Dampfdruck bei der jeweiligen Temperatur angibt. Offenbar hängt der Ruheradius $R_{\mathrm{n}}$ linear vom Dampfdruck $p_{\mathrm{v}}$ ab.

Der Grund für den zu großen Blasenradius in den späteren Rebounds bei Gilmore und Eick ist offenbar, dass in diesen Modellen keine Phasenübergänge (z. B. Kondensation) 




Abbildung 3.15: Bildsequenz vom 1. Kollaps einer lasererzeugten Blase bei $T=30.5^{\circ} \mathrm{C}$. Bildabstand: $3.3 \mu$ s. Das erste Bild wurde $96.7 \mu$ s nach dem Laserdurchbruch aufgenommen.

stattfinden können, diese aber im Experiment auftreten. Die Diffusion von Gas über die Blasenwand wird in allen drei Modellen vernachlässigt.

Außerdem ist die Stoßwellenabstrahlung in diesem Modell nicht exakt berechnet. Fuster et al. [17] und Koch et al. [18] vergleichen die maximalen Drücke der Stoßwellen sowie die Blasenradien im Rebound, die sich aus dem Gilmore Modell ergeben, mit den Ergebnissen einer Finite Volumen Simulation. Dabei zeigte sich, dass diese beiden Modelle sich vor allem bei heftigen Kollapsen unterscheiden, also wenn das Verhältnis $R_{\mathrm{n}} / R_{\max }$ klein ist. Für die Dynamik von Blasen mit hohem Dampfanteil ist dieser Unterschied vermutlich vernachlässigbar.

In der Finite Volumen Simulation kann der Druck in der Blase inhomogen sein. Im Gilmore Modell (und auch bei Eick und Toegel) ist der Druck in der Blase homogen. Die daraus resultierende Abweichung im Blasenradius ist allerdings klein [18].

Wenn der Ruheradius nicht angepasst wird, können Gilmore und Eick Modell die stärkeren Rebounds bei hohen Wassertemperaturen nicht wiedergeben. Ein höherer Dampfdruck verlängert die Kollapszeit, ändert aber den maximalen Radius im Rebound kaum (siehe Abschnitt 3.2.4).

\subsection{Ausblick}

Möglicherweise ist durch den schwächeren Kollaps, also die vergleichsweise großen minimalen Radien im Kollaps, bei hohen Wassertemperaturen die Erosion weniger stark.

Laut Prosperetti und Lezzi [15] ist das Gilmore Modell dem Keller-Miksis Modell überlegen, da in den Differentialgleichungen die Enthalpie anstatt des Drucks verwendet wird. Wenn man also das Toegel Modell auf der Gilmore Gleichung aufbaut, erhält man vermutlich eine bessere Übereinstimmung mit den Messdaten. 

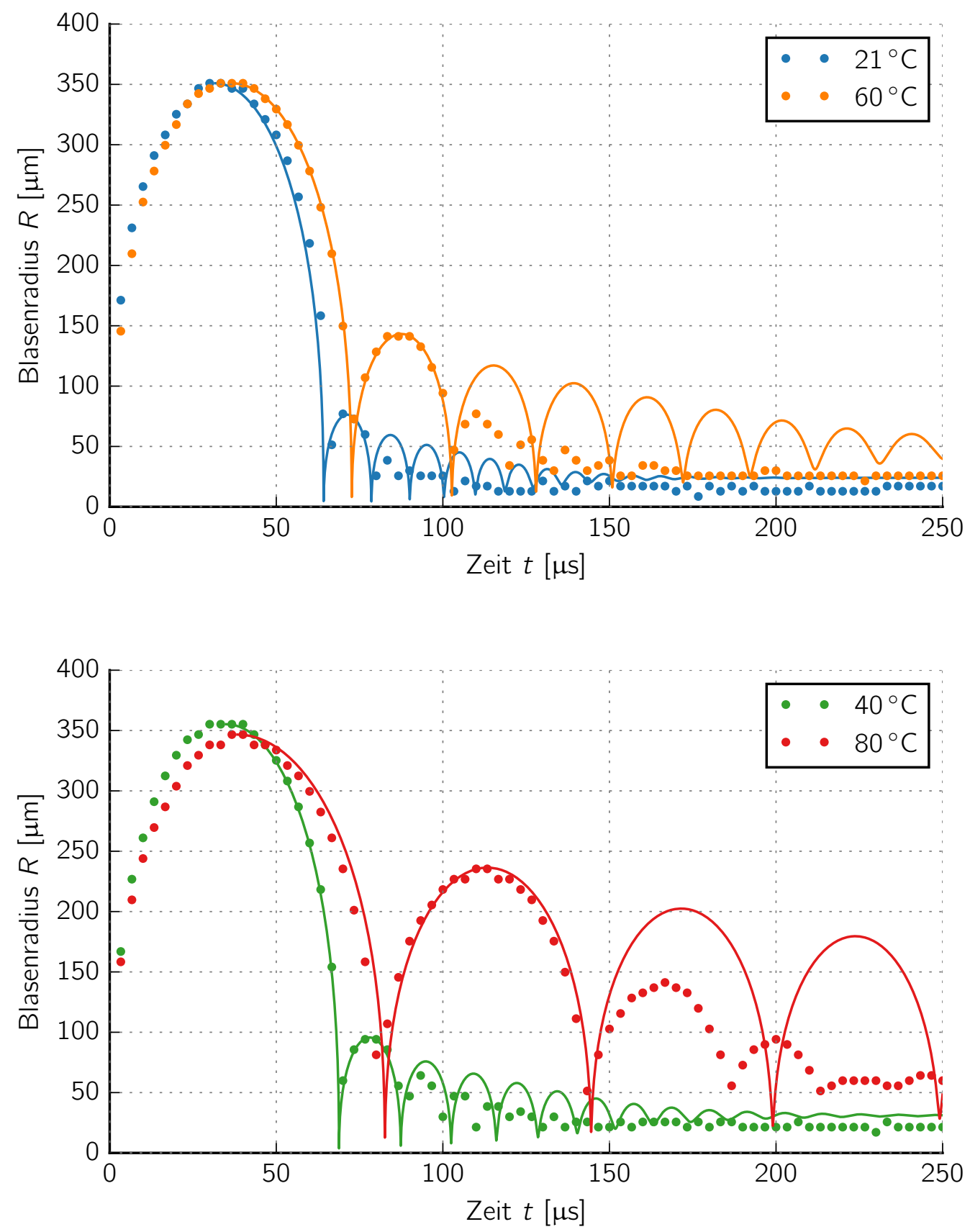

Abbildung 3.16: Blasenradius $R$ als Funktion der Zeit $t$ bei verschiedenen Wassertemperaturen. Vergleich der experimentellen Ergebnisse (Punkte) mit den numerischen Rechnungen nach dem Eick Modell (durchgezogene Linien). 


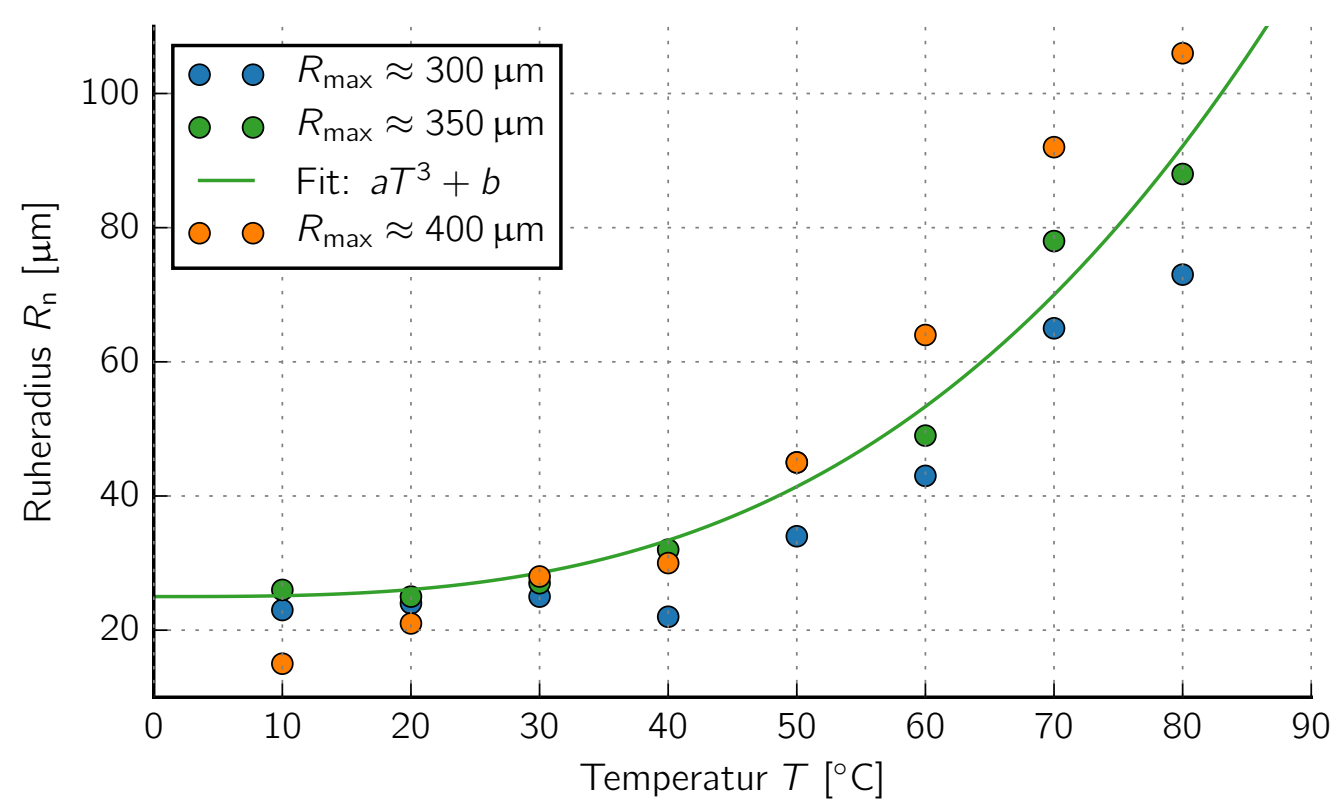

Abbildung 3.17: Ruheradius $R_{\mathrm{n}}$ als Funktion der Temperatur $T$. Der Ruheradius wurde jeweils durch Anpassung des Eick Modells an die Messdaten ermittelt. Die durchgezogene Linie stellt eine kubische Anpassung dar mit den Parametern $a=1.3 \cdot 10^{-4} \mu \mathrm{m}\left({ }^{\circ} \mathrm{C}\right)^{-3}$ und $b=25.0 \mu \mathrm{m}$.

Interessant wäre auch ein Vergleich des Modells von Dreyer et al. [30] mit experimentellen Ergebnissen. 




Abbildung 3.18: Auftragung des Ruheradius $R_{\mathrm{n}}$ gegenüber dem Dampfdruck für verschiedene Temperaturen. Der Ruheradius wurde jeweils durch Anpassung des Eick Modells an die Messdaten ermittelt. Die durchgezogene Linie stellt eine lineare Anpassung dar mit den Parametern $m=151.4 \cdot 10^{-3} \mu \mathrm{mbar}^{-1}$ und $c=21.5 \mu \mathrm{m}$. 

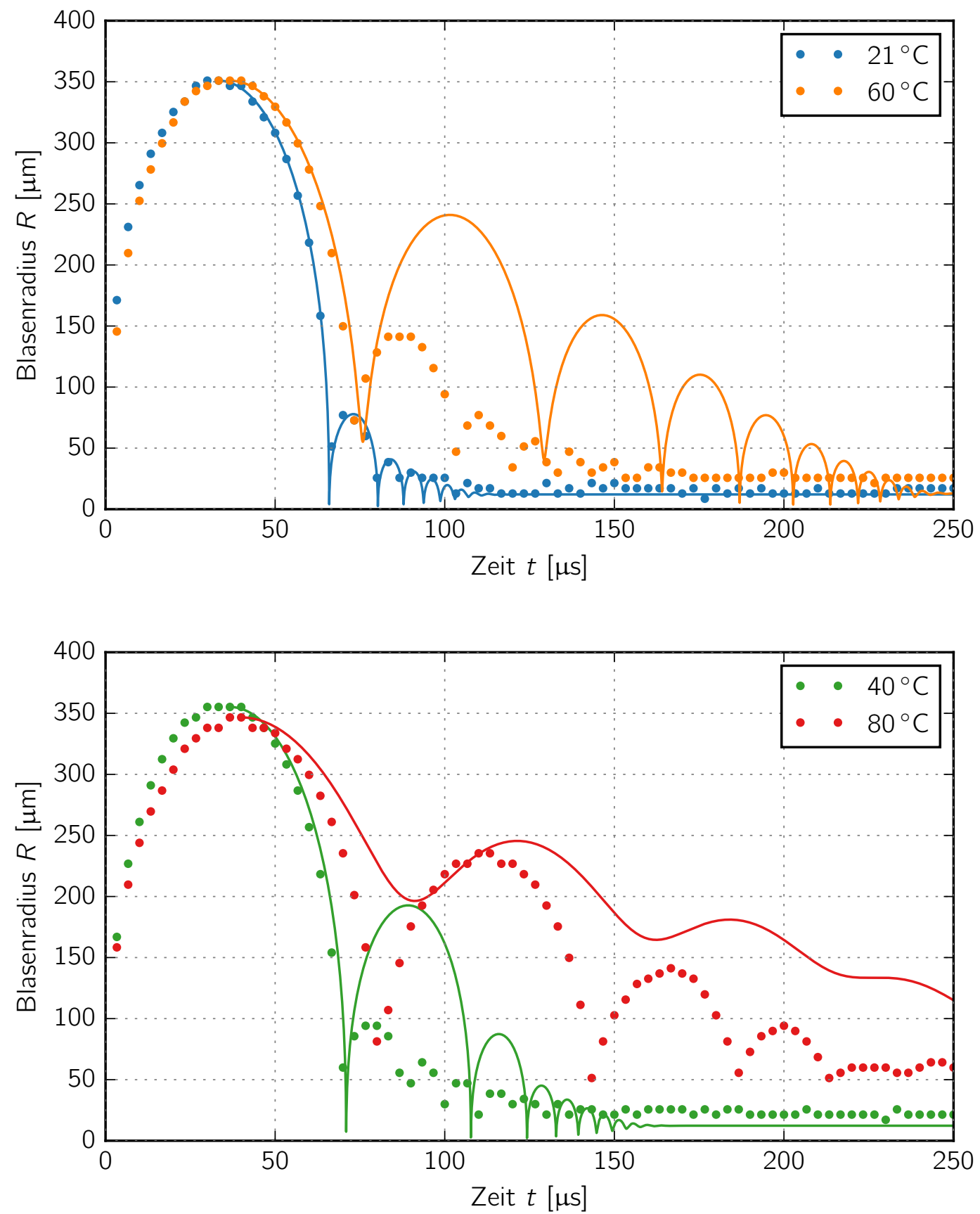

Abbildung 3.19: Blasenradius $R$ als Funktion der Zeit $t$ bei verschiedenen Wassertemperaturen. Vergleich der experimentellen Ergebnisse (Punkte) mit den numerischen Rechnungen nach dem Toegel Modell (durchgezogene Linien). Ruheradius in allen Rechnungen: $R_{\mathrm{n}, \mathrm{g}}=12 \mu \mathrm{m}$. 



\section{Temperaturmessung mittels laserinduzierter Fluoreszenz}

Im Kollaps erwartet man aufgrund der hohen Temperaturen im Blaseninneren einen Anstieg der Wassertemperatur in einer dünnen Schicht um die Blase (thermische Grenzschicht). Wenn die Blase ihren Maximalradius erreicht, kann die Flüssigkeit dagegen abgekühlt werden, da Druck und Temperatur in der Blase sinken und Wasser an der Grenzfläche verdampft (latente Wärme).

Für eine typische Kollapszeit von $t=60 \mu$ s ergibt sich mit der Temperaturleitfähigkeit $\alpha$ von Wasser eine Grenzschichtdicke von $d=\sqrt{\alpha t} \approx 3 \mu \mathrm{m}$. Es stellt sich nun die Frage, wie groß die Temperaturänderung innerhalb der thermischen Grenzschicht ist. Die Temperaturdifferenz $\Delta T$ hängt wie folgt mit der Energieänderung $\Delta Q$ zusammen:

$$
\begin{aligned}
c_{p} & =\frac{\Delta Q}{m \Delta T} \\
\Rightarrow \Delta Q & =c_{p} m \Delta T
\end{aligned}
$$

Dabei ist $c_{p}$ die spezifische Wärmekapazität von Wasser (bei konstantem Druck) und $m=\varrho V$ die Masse. Das Volumen der thermischen Grenzschicht beträgt $V=4 \pi R^{2} d$. Bei einem Blasenradius von $R=50 \mu \mathrm{m}$ (Blase im Kollaps) führt eine Energieänderung von $\Delta Q=10 \mu \mathrm{J}$ innerhalb der Grenzschicht zu einem Temperaturanstieg von $\Delta T=$ $25.4 \mathrm{~K}$.

Die Laserpulsenergie für die Blasenerzeugung beträgt in diesem Experiment wenige Millijoule. Tatsächlich wird nur ein sehr kleiner Teil der Energie in Wärme umgewandelt. Die restliche Energie wird beim Laserdurchbruch als Stoßwelle abgestrahlt, bewirkt in Form von mechanischer Energie das Anwachsen der Blase, führt zur Verdampfung von Wasser und wird in Plasmastrahlung umgewandelt [62]. Zur Blasenerzeugung ist nur ein einzelner Laserpuls nötig. Nimmt man an, dass ein Laserpuls eine Energie von $1 \mathrm{~mJ}$ hat und $10 \%$ davon in Wärme umgewandelt werden, so ergibt sich die oben bereits berechnete Temperaturerhöhung in der Grenzschicht von $\Delta T=25.4 \mathrm{~K}$.

In früheren Experimenten an einzelnen Blasen konnten schon Temperaturänderungen 
sichtbar gemacht und gemessen werden. Eine experimentelle Arbeit von Vogel und Lauterborn [63] behandelt zwar nicht die Messung der Temperatur, es konnten aber Temperaturschichtungen zur Strömungsvisualisierung nutzbar gemacht werden. Die Autoren haben in einer wassergefüllten Küvette ein Temperaturgefälle (oben $50^{\circ} \mathrm{C}$, unten $10^{\circ} \mathrm{C}$ ) eingestellt und darin die Jetströmung einer asphärisch kollabierenden Laserblase mit dem Dunkelfeldverfahren sichtbar gemacht. Dabei konnten noch Temperaturdifferenzen von $0.5^{\circ} \mathrm{C}$ zwischen Jet und umgebender Flüssigkeit beobachtet werden.

Temperaturmessungen an größeren Blasen $\left(R_{\max } \approx 10 \mathrm{~mm}\right)$ mit einer Infrarotkamera sind von Dular und Coutier-Delgosha [64] durchgeführt worden. Die Blasen waren dabei dicht vor einer festen Wand $(\gamma \leq 1.3)$ und es wurde in der Nähe der Blase ein Temperaturanstieg von bis zu $3{ }^{\circ} \mathrm{C}$ gemessen. Aufgrund der relativ starken Absorption von Infrarotstrahlung in Wasser kann die Temperatur mit dieser Methode nur dicht hinter einem Fenster in der Küvettenwand gemessen werden.

Quinto-Su et al. [65] haben Temperaturmessungen mit einem Fluoreszenzfarbstoff an lasererzeugten Mikroblasen $\left(R_{\max } \approx 10-35 \mu \mathrm{m}\right)$ in einem $19 \mu \mathrm{m}$ hohen Mikrokanal sowie in einem nach oben offenen Behälter durchgeführt. In beiden Fällen wurde die Blase etwa $2 \mu \mathrm{m}$ oberhalb der unteren Wand erzeugt $(\gamma \leq 0.2)$. Der Farbstoff wurde mit einer Quecksilberdampflampe angeregt und es konnte ein Temperaturanstieg von bis zu $12{ }^{\circ} \mathrm{C}$ gemessen werden, da hier ein sehr kleines Volumen erwärmt wird.

Im Unterschied zu früheren Experimenten haben die in diesem Kapitel untersuchten Blasen einen maximalen Radius von etwa $500 \mu \mathrm{m}$ und werden in der Mitte einer relativ großen Küvette $(30 \mathrm{~mm} \times 30 \mathrm{~mm} \times 30 \mathrm{~mm})$ erzeugt, so dass sie frei schwingen können (keine Wand in der Nähe). Die LIF-Methode zur Temperaturmessung wurde ausgehend von [66] an die Temperaturmessung an einzelnen Blasen angepasst. Die zentrale Fragestellung dieses Kapitels ist: Wieviel Wärme wird im Kollaps von der Blase an die umliegende Flüssigkeit abgegeben?

Dazu wird ein Experiment zur Temperaturmessung mittels laserinduzierter Fluoreszenz (LIF) an kollabierenden Blasen beschrieben. Die Temperaturmessungen sollen Aufschluss über den Wärmetransport über die Grenzfläche Blase-Flüssigkeit geben. Zunächst wird das LIF-Prinzip vorgestellt und die Auswahl der Fluoreszenzfarbstoffe erläutert. Der nächste Abschnitt beschäftigt sich mit Vorexperimenten und Tests der Temperaturmessung mit laserinduzierter Fluoreszenz. Schließlich werden LIF-Messungen an einzelnen lasererzeugten Blasen sowie an einzelnen im Schallfeld gefangenen Blasen beschrieben. Numerische Modelle dienen zur Abschätzung des Temperaturprofils in der Grenzschicht um die Blase. 


\subsection{Prinzip der laserinduzierten Fluoreszenz}

Laserinduzierte Fluoreszenz ist eine nicht-invasive Methode, mit der u. a. die Temperatur in Flüssigkeiten gemessen werden kann [67]. Dazu wird ein Fluoreszenzfarbstoff in einer Flüssigkeit gelöst und durch einen Laser angeregt. Einen Teil der dabei absorbierten Energie gibt der Farbstoff unter Emission von Photonen spontan wieder ab. Da auch strahlungslose Übergänge stattfinden können und dadurch ein Teil der Energie verbraucht wird, ist die Wellenlänge des Fluoreszenzlichts größer als die Anregungswellenlänge. Bei Farbstoffen mit temperaturabhängiger Fluoreszenz ändert sich das Emissionsspektrum des Fluoreszenzlichts mit der Temperatur. Dabei hängt vor allem die Fluoreszenzintensität von der Temperatur ab. Wenn vorher eine Kalibrierung durchgeführt wird, kann man also die Temperatur aus der gemessenen Intensität des Fluoreszenzlichts bestimmen.

Bei einfallendem Licht mit einer Intensität $I_{\text {in }}$ beträgt die Intensität des emittierten Fluoreszenzlichts

$$
I=I_{\text {in }} C \phi \varepsilon \text {. }
$$

Dabei ist $C$ die Konzentration, $\phi$ die Quanteneffizienz und $\varepsilon$ der Absorptionskoeffizient des Farbstoffs. Bei den meisten Farbstoffen hängt nur die Quanteneffizienz von der Temperatur ab; bei manchen auch der Absorptionskoeffizient [66]. Bei fester Intensität $I_{\text {in }}$ und fester Konzentration $C$ kann man also die Temperatur aus der Fluoreszenzintensität bestimmen, die sich mit $\phi \varepsilon$ ändert.

Da es praktisch immer Schwankungen in der Anregungsintensität $I_{\text {in }}$ gibt, sollte man einen zusätzlichen Farbstoff verwenden, der eine sehr schwache Temperaturabhängigkeit aufweist. Damit lässt sich die Genauigkeit der Temperaturmessung erhöhen [66]. Das Verhältnis der Intensitäten $I_{A}$ und $I_{B}$ der beiden Farbstoffe

$$
\frac{I_{A}}{I_{B}}=\frac{C_{A} \phi_{A} \varepsilon_{A}}{C_{B} \phi_{B} \varepsilon_{B}}
$$

ist unabhängig von der Anregungsintensität $I_{\text {in }}$. Es kann allerdings zu einem Übersprechen kommen (siehe Abschnitt 4.4.2).

Für die LIF-Experimente werden Fluoreszenzfarbstoffe verwendet, die wasserlöslich sind und sich mit Laserlicht der Wellenlänge $532 \mathrm{~nm}$ anregen lassen. Als temperaturabhängiger Farbstoff wird Rhodamin B (RhodB) verwendet, dessen Fluoreszenzintensität mit steigender Temperatur abnimmt. Um die Genauigkeit der Temperaturmessung zu erhöhen, wird als zusätzlicher Farbstoff Sulforhodamin 101 (SR101) verwendet, der nur eine sehr schwache Temperaturabhängigkeit aufweist. 
Im Emissionsspektrum von Rhodamin B in Wasser tritt ein Maximum bei etwa $560 \mathrm{~nm}$ auf. Für Sulforhodamin 101 in Wasser liegt das Maximum bei etwa $600 \mathrm{~nm}$. Abbildung 4.1 zeigt die überlagerten Spektren beider Farbstoffe in einer gemeinsamen Lösung bei verschiedenen Temperaturen. Sie wurden bei einer Anregungswellenlänge von $532 \mathrm{~nm}$ (Linienbreite: $5 \mathrm{~nm}$ ) mit einem Spektrometer vom Typ Jasco FP-6500 aufgenommen.

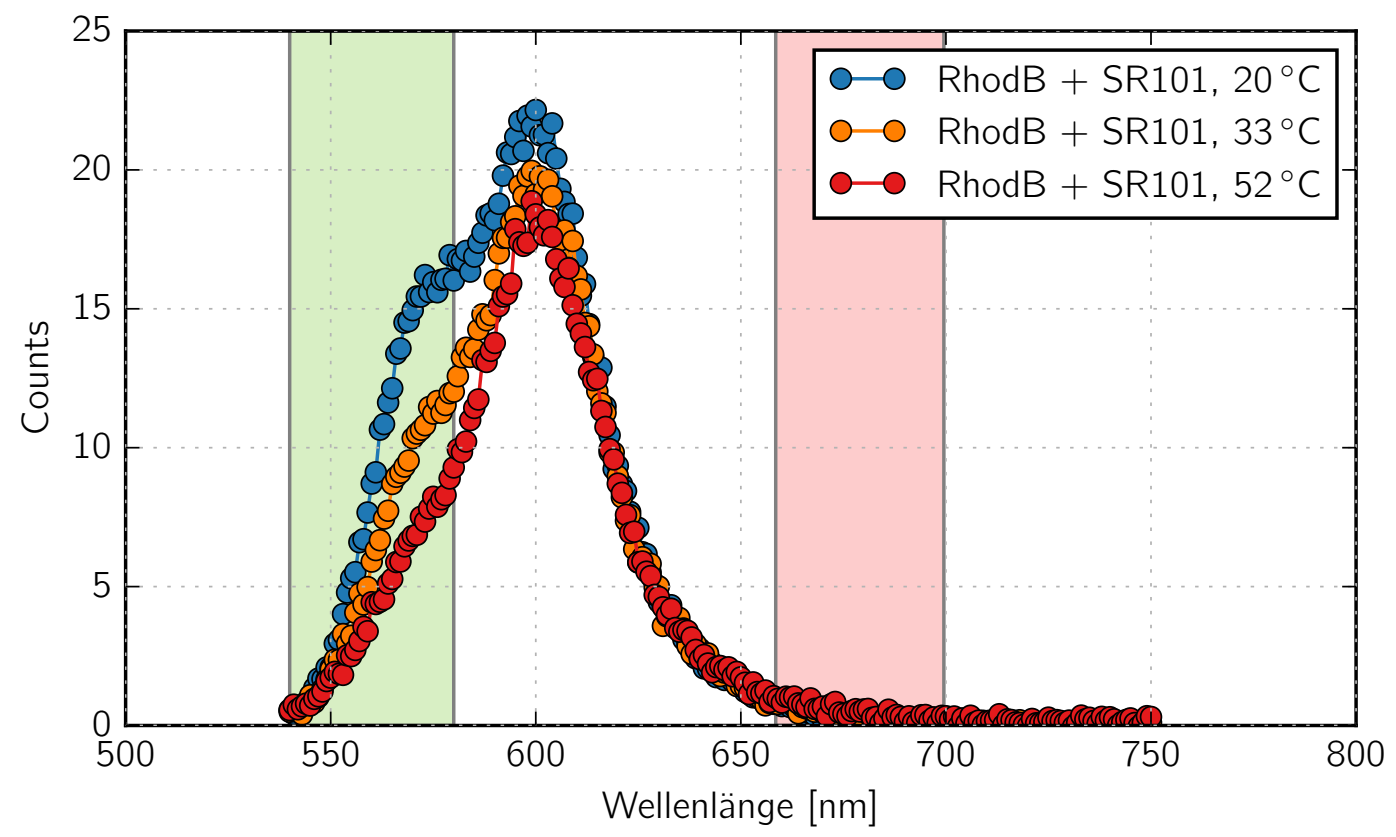

Abbildung 4.1: Gemessene Emissionsspektren von Rhodamin B $\left(C=1.0 \cdot 10^{-8} \mathrm{~mol} / \mathrm{l}\right)$ und Sulforhodamin $101\left(C=1.6 \cdot 10^{-8} \mathrm{~mol} / \mathrm{l}\right)$ in deionisiertem Wasser bei verschiedenen Temperaturen. Die farbig markierten Wellenlängenbereiche werden von den Bandpassfiltern an den Kameras durchgelassen.

Da die verwendeten Farbstoffe bei einer Wellenlänge von $532 \mathrm{~nm}$ effizient anregbar sind und Emissionsmaxima deutlich oberhalb davon aufweisen, können Anregungslaserlicht und Fluoreszenzlicht leicht voneinander getrennt werden. 


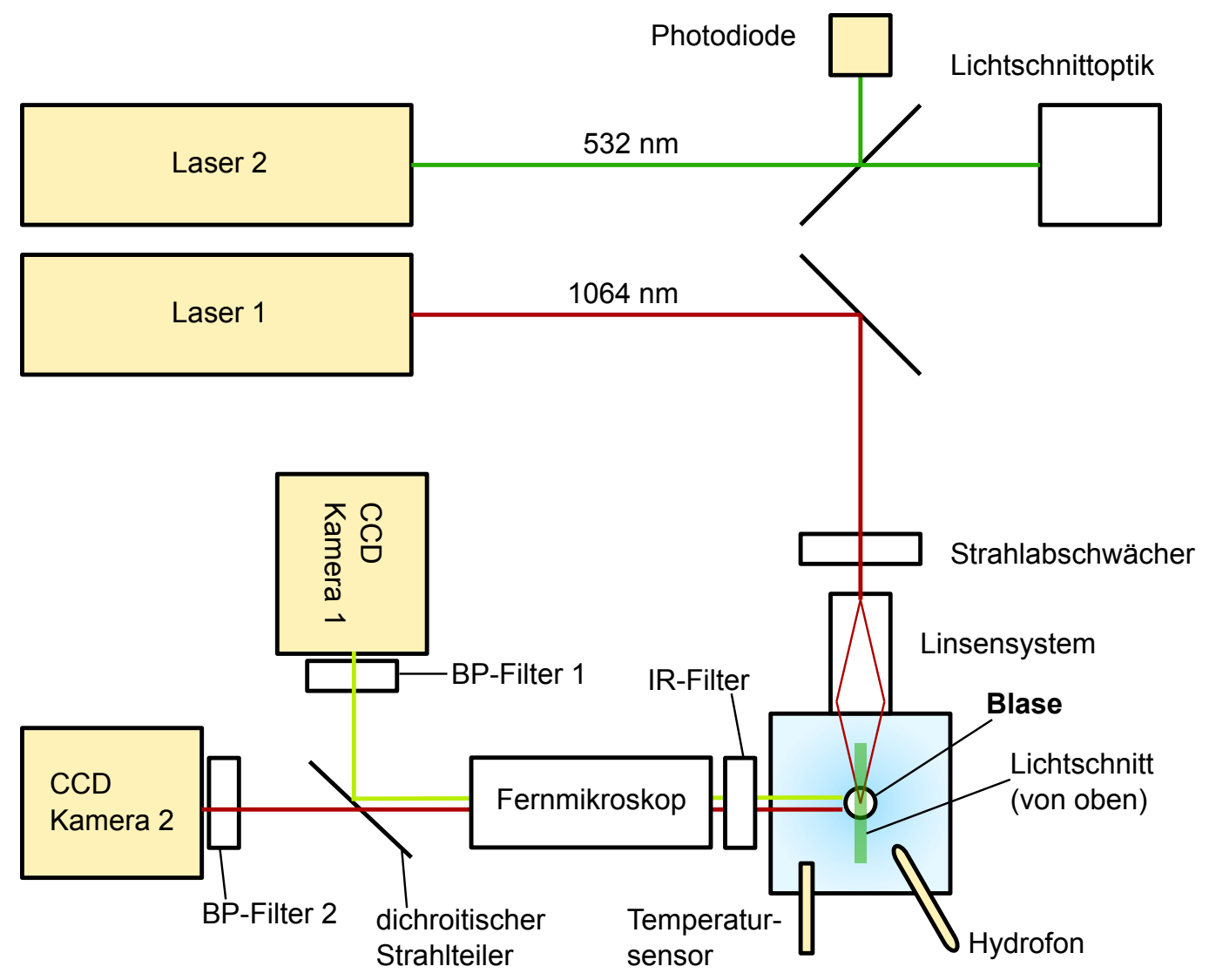

Abbildung 4.2: Experimenteller Aufbau zur Temperaturmessung an einer laserinduzierten Blase. Die beiden Nanosekundenlaser dienen zur Blasenerzeugung (Laser 1) und zur Anregung der Farbstoffe (Laser 2). Der dichroitische Strahlteiler lenkt das Fluoreszenzlicht auf die beiden CCDKameras, an denen zusätzlich jeweils ein Bandpassfilter montiert ist. Der IR-Filter blockiert das infrarote Licht von Laser 1. 


\subsection{Experimenteller Aufbau}

Der experimentelle Aufbau zur Temperaturmessung an einzelnen Blasen ist in Abb. 4.2 dargestellt. Laser 1 (Spectra Physics Quanta Ray PIV-400, $\lambda=1064 \mathrm{~nm}$, Pulsdauer: $8 \mathrm{~ns}$ ) erzeugt wie in Abschnitt 2.4.1 beschrieben eine einzelne Blase in der Farbstofflösung. Das Absorptionsmaximum der Farbstoffe liegt im grünen Wellenlängenbereich, dieser Laser regt sie daher nicht an.

Mit einem Puls aus Laser 2 (Litron nano, $\lambda=532 \mathrm{~nm}$, Pulsdauer: $4 \mathrm{~ns}$ ) wird ein Lichtschnitt erzeugt, der die Farbstoffe anregt. Auf diese Weise kann die räumliche Temperaturverteilung in einer dünnen Flüssigkeitsschicht in einem Zeitfenster von wenigen Nanosekunden (Laserpulsdauer, Fluoreszenzlebensdauer) gemessen werden. Dazu lenkt ein Spiegelarm der Firma Dantec das Laserlicht so um, dass es von oben in die Küvette fällt. Am Ende des Spiegelarms befindet sich eine Zylinderlinse ( $f=250 \mathrm{~mm}$, plankonvex), die sich mit Hilfe von Verschiebetischen in alle drei Raumrichtungen bewegen lässt.

Die Küvette ist gefüllt mit einer Lösung von Rhodamin B mit einer Konzentration von $1 \cdot 10^{-6} \mathrm{~mol} / \mathrm{l}$ und Sulforhodamin $101 \mathrm{mit}$ einer Konzentration von $2 \cdot 10^{-6} \mathrm{~mol} / \mathrm{l}$ in deionisiertem Wasser. Die Lösung weist einen $\mathrm{pH}-$ Wert von 7 auf und ist damit neutral. Das Fluoreszenzlicht wird mit einem Fernmikroskop (Infinity K2) aufgenommen. Ein dichroitischer Strahlteiler (Transmission $605 \mathrm{~nm}$ bis $870 \mathrm{~nm}$ (SR101), Reflexion $450 \mathrm{~nm}$ bis $580 \mathrm{~nm}(\mathrm{RhodB}))$ trennt das Fluoreszenzlicht der beiden Farbstoffe voneinander und lenkt es auf die beiden Kameras $(2 \times$ PCO SensiCam qe). Die Belichtungszeit beträgt $5 \mu$ s und ist damit wesentlich länger als die Fluoreszenzlebensdauer der Farbstoffe. Die Dauer einer einzelnen Temperaturmessung wird daher durch die Fluoreszenzlebensdauer, die wenige Nanosekunden beträgt, bestimmt. Zusätzlich ist an jeder Kamera ein Bandpassfilter (BP-Filter 1: ET 560/40 und BP-Filter 2: HC 679/41, siehe Abb.4.2) montiert, so dass nur Licht mit den in Abb. 4.1 markierten Wellenlängenbereichen durchgelassen wird. Ein zusätzlicher Infrarotfilter (IR-Filter: Kurzpass mit Grenzwellenlänge $750 \mathrm{~nm}$ ) vor dem Fernmikroskop sorgt dafür, dass das Laserlicht zur Blasenerzeugung $(\lambda=1064 \mathrm{~nm})$ nicht in die Kameras gelangt.

Die Verzögerungszeit zwischen dem Puls aus Laser 1 (Blasenerzeugung) und dem Puls aus Laser 2 (Lichtschnitt) sowie zwischen Laserpuls 1 und dem Auslösen der beiden Kameras kann mit einem Delaygenerator (Stanford Research Systems DG535) präzise eingestellt werden.

Bei der Belichtung können mehrere Pixel auf dem CCD-Sensor der Kamera zusammengefasst werden (Binning), um ein stärkeres Signal zu erhalten. Einige Messungen wur- 
den mit Binning $2 \times 2$ gemacht.

Die Temperatur der Farbstofflösung wird am Rand der Küvette mit einem Pt100-Sensor gemessen. Außerdem befindet sich noch ein Nadelhydrofon in der Küvette, um die Stoßwellen aufzunehmen, die bei der Erzeugung und beim Kollaps von der Blase ausgehen. Die Pulsenergie von Laser 2 (Anregung der Farbstoffe) wird mit einem Photodetektor überwacht.

Zur Überprüfung der Lichtschnittdicke kann man die Zylinderlinse um $90^{\circ}$ drehen (Blickrichtung dann in der Lichtschnittebene). Für die eigentliche Messung wird die Zylinderlinse so gedreht, dass die optische Achse der Abbildungsoptik (Kameras und Fernmikroskop) senkrecht zur Lichtschnittebene verläuft.

Vor der eigentlichen Messung muss eine Kalibrierung durchgeführt werden. Dazu wird die Temperatur der Farbstofflösung in der Küvette mit einem Thermostaten geregelt und es werden verschiedene Temperaturen im Bereich von $18^{\circ} \mathrm{C}$ bis $37^{\circ} \mathrm{C}$ eingestellt. Die Temperatur wird mit dem Pt100-Sensor gemessen und das Fluoreszenzlicht wird mit den beiden Kameras aufgenommen. So ergibt sich eine Abhängigkeit der Fluoreszenzintensität von der Temperatur.

\subsection{Auswertung}

Die aufgenommenen Bilder werden mit Hilfe von Matlab und Python ausgewertet. Als erstes wird von jedem aufgenommenen Kamerabild ein Dunkelbild abgezogen. (Der Rauschpegel der Kameras liegt bei etwa 40 Counts von max. $4096=2^{12}$.) Es ist praktisch unmöglich, die Kameras so auszurichten, dass beide genau den gleichen Bildausschnitt aufnehmen. Daher wird mit beiden Kameras ein Punktgitter aufgenommen und die beiden Bilder werden durch Verschiebung, Drehung und Skalierung zur Deckung gebracht. Die Transformationen werden auf die Bilder von Kamera 1 (RhodB) angewendet; die Bilder von Kamera 2 (SR101) bleiben unverändert.

Für die Kalibrierung werden die bei verschiedenen Temperaturen aufgenommenen Bilder ausgewertet. Durch Mittelung über einen Ausschnitt erhält man aus jedem Bild einen mittleren Intensitätswert. Aus den mittleren Intensitäten beider Kamerabilder wird jeweils das Verhältnis

$$
\frac{\bar{I}_{\text {RhodB }}}{\bar{I}_{\text {SR101 }}}
$$


gebildet und gegen die Temperatur aufgetragen. Dabei ergibt sich ein linearer Zusammenhang. Durch Anpassung der Funktion

$$
T\left(\bar{I}_{\mathrm{RhodB}}, \bar{I}_{\mathrm{SR} 101}\right)=p_{1} \frac{\bar{I}_{\mathrm{RhodB}}}{\bar{I}_{\mathrm{SR} 101}}+p_{2}
$$

an die Messdaten erhält man die Parameter $p_{1}$ und $p_{2}$, mit deren Hilfe später aus dem Intensitätsverhältnis die Temperatur bestimmt werden kann.

Die Intensitätsverteilung des Lichtschnitts, und damit auch die Intensität des Fluoreszenzlichts, ist nicht homogen. Daher werden am Anfang einer Messreihe einige Referenzbilder aufgenommen, die die ungestörte Intensitätsverteilung ohne das zu untersuchende Objekt zeigen. Die anschließend aufgenommenen LIF-Bilder werden durch diese Referenzverteilung $I_{0, \operatorname{RhodB}}(x, y)$ bzw. $I_{0, \mathrm{SR} 101}(x, y)$ geteilt.

Bei der Auswertung der LIF-Messungen an Blasen wird für jedes Bilderpaar (Kamera 1 und 2) pixelweise das Verhältnis

$$
\frac{I_{\mathrm{RhodB}}(x, y)}{I_{\mathrm{SR} 101}(x, y)}
$$

gebildet.

Die Umrechnung der Intensität $I$ in eine Temperatur $T$ erfolgt wie in [68] beschrieben:

$$
T(I(x, y))=T_{\mathrm{c}}\left(I_{\mathrm{c}}\left(T_{\mathrm{ref}}\right) \frac{I(x, y)}{I_{\mathrm{ref}}}\right)
$$

Dabei ist entweder

$$
I(x, y)=\frac{I_{\mathrm{RhodB}}(x, y)}{I_{0, \operatorname{RhodB}}(x, y)} \quad \text { (einzelner Farbstoff) }
$$

oder

$$
I(x, y)=\frac{I_{\mathrm{RhodB}}(x, y)}{I_{0, \mathrm{RhodB}}(x, y)} \frac{I_{0, \mathrm{SR} 101}(x, y)}{I_{\mathrm{SR} 101}(x, y)} \quad \text { (zwei Farbstoffe) }
$$

Eine Kalibrierung vor der eigentlichen Messung liefert die Abhängigkeit $I_{\mathrm{c}}\left(T_{\mathrm{c}}\right)$ sowie die Umkehrung $T_{\mathrm{c}}\left(I_{\mathrm{c}}\right)$. Die Intensität $I_{\text {ref }}$ ergibt sich aus einem Bildausschnitt, in dem die bekannte Temperatur $T_{\text {ref }}$ herrscht, wobei $T_{\text {ref }}$ mit einem Pt100-Sensor gemessen wird. 
4.4 Vorexperimente, Testen der LIF-Methode

4.4.1 Messung der Lichtschnittdicke

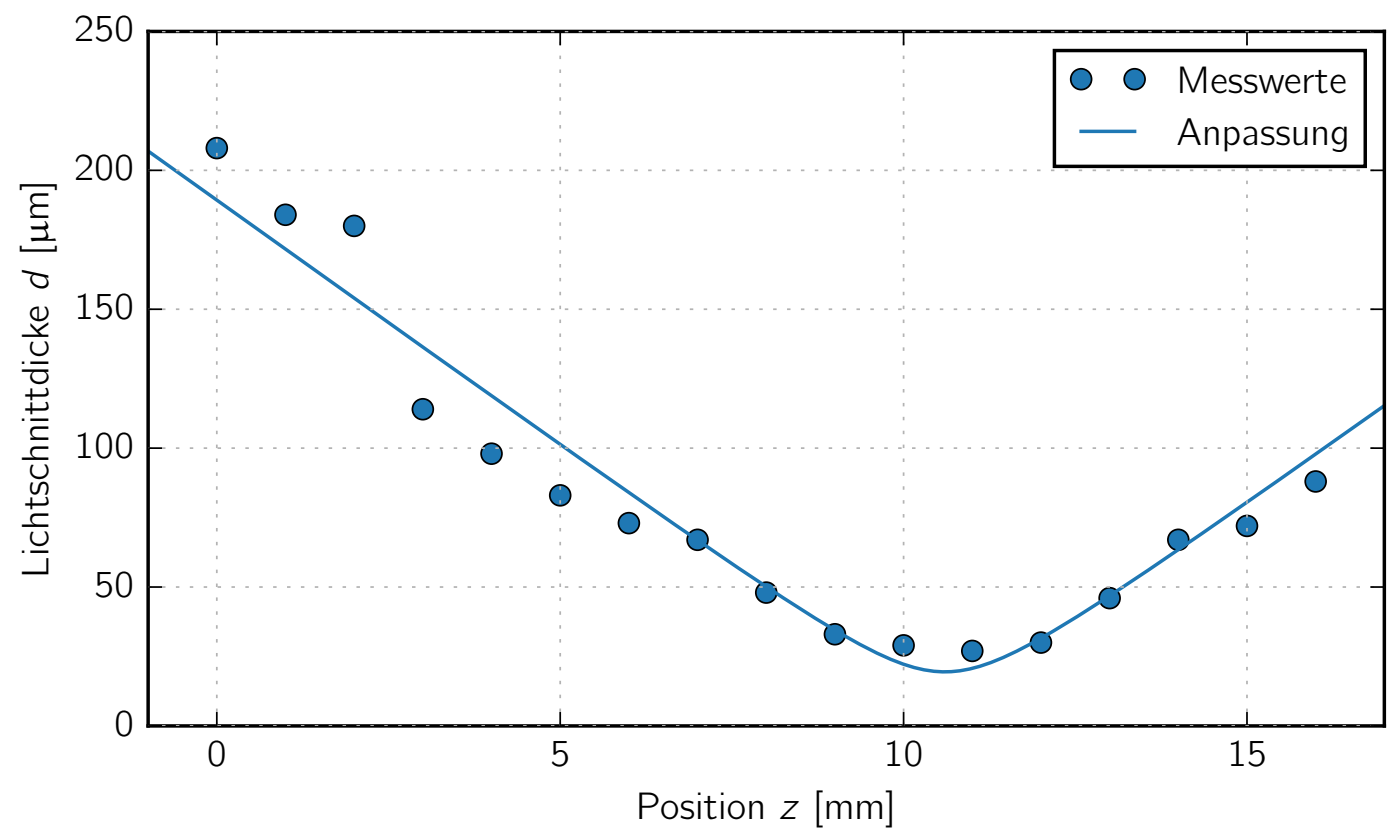

Abbildung 4.3: Messung der Lichtschnittdicke in Luft mit einem Laser Beam Profiler in verschiedenen Abständen zur Zylinderlinse. Die minimale Lichtschnittdicke beträgt $27 \mu \mathrm{m}$ (bei $z=11 \mathrm{~mm}$ ).

Um eine möglichst kleine Lichtschnittdicke zu erreichen, muss der Abstand der Lichtschnittoptik zur Küvette optimiert werden. Betrachtet man den Lichtschnitt als Gaußschen Strahl, so gibt es eine Position (die Strahltaille), an der der Strahldurchmesser minimal ist. Der optimale Abstand ergibt sich durch eine Messung der Lichtschnittdicke (FWHM) in Luft in verschiedenen Abständen von der Zylinderlinse $(f=250 \mathrm{~mm}) \mathrm{mit}$ einem Laser Beam Profiler (Metrolux BeamLux II ML 1200). Dabei wird der Abstand relativ gemessen, d.h. es wird jeweils die Position des Beam Profilers bestimmt (nicht der absolute Abstand der Linse zum Beam Profiler).

Wie in Abb. $4.3 \mathrm{zu}$ sehen ist, wird die Lichtschnittdicke an der Position $z=11 \mathrm{~mm}$ mit 
$d=27 \mu \mathrm{m}$ minimal. Die Funktion

$$
w(z)=w_{0} \sqrt{1+\frac{\left(z-z_{\min }\right)^{2}}{z_{0}^{2}}}
$$

gibt den Radius eines Gaußschen Strahls entlang der optischen Achse ( $z$-Achse) in der Nähe der Strahltaille an [69]. Eine Anpassung dieser Funktion an die Messwerte (siehe Abb. 4.3) liefert die Rayleighlänge $z_{0}=1.1 \mathrm{~mm}$, die minimale Lichtschnittdicke $w_{0}=$ $19.5 \mu \mathrm{m}$ sowie die Position des Minimums $z_{\min }=10.6 \mathrm{~mm}$.

4.4.2 Überprüfung der Bandpassfilter, Übersprechen

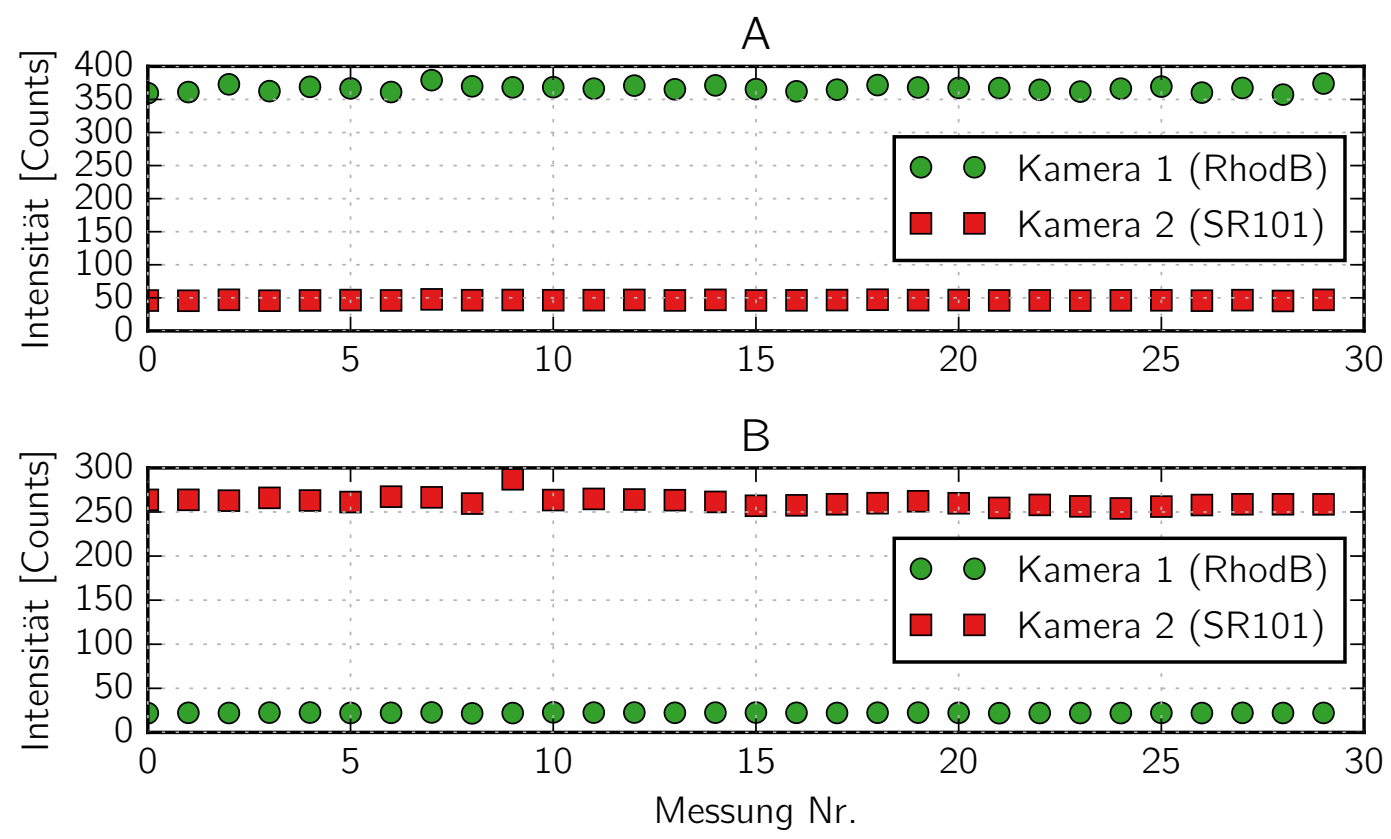

Abbildung 4.4: Test des Übersprechens durch Aufnahme des Fluoreszenzlichts jeweils eines Farbstoffs mit beiden Kameras bei Raumtemperatur. Rhodamin B Lösung $\left(C=1 \cdot 10^{-6} \mathrm{moll}^{-1}\right)(\mathrm{A})$, Sulforhodamin 101 Lösung $(C=$ $\left.2 \cdot 10^{-6} \mathrm{moll}^{-1}\right)(\mathrm{B})$.

Zur Überprüfung der Bandpassfilter wird jeder Farbstoff einzeln in deionisiertem Wasser gelöst und das Fluoreszenzlicht mit beiden Kameras aufgenommen. Idealerweise lässt der Bandpassfilter an Kamera 1 nur Fluoreszenzlicht von Rhodamin B und der 
Bandpassfilter an Kamera 2 nur Fluoreszenzlicht von Sulforhodamin 101 durch. Jede Messung liefert zwei Kamerabilder (eins für jeden Farbstoff). Eine Mittelung über das gesamte Kamerabild ergibt jeweils einen Wert, der proportional zur Fluoreszenzintensität ist.

Wie in Abb. 4.4zu erkennen ist, gelangt auch Fluoreszenzlicht vom jeweils anderen Farbstoff in die Kamera. Wenn die Farbstofflösung nur Rhodamin B enthält, ergibt sich das Verhältnis $I_{\text {Kamera1 }} / I_{\text {Kamera2 }}=7.9$. Bei einer reinen Sulforhodamin 101 Lösung ergibt sich $I_{\text {Kamera2 }} / I_{\text {Kamera1 }}=11.8$. Das Fluoreszenzlicht der beiden Farbstoffe lässt sich also in dem beschriebenen Aufbau ausreichend voneinander trennen.

\subsubsection{Kalibrierung}

Die Durchführung der Kalibrierung wurde bereits in Abschnitt 4.2 beschrieben. Für jede eingestellte Temperatur werden 30 Messungen gemacht, jede Messung liefert zwei Kamerabilder. Nun wird wie in Abschnitt 4.3 beschrieben jeweils über einen Ausschnitt des Kamerabildes gemittelt, so dass sich ein Wert für die Fluoreszenzintensität ergibt.

In Abb. 4.5 oben sind die bei verschiedenen Temperaturen gemessenen Fluoreszenzintensitäten für jeden Farbstoff einzeln aufgetragen. Es ist deutlich zu erkennen, dass im Fall von Rhodamin $B$ die Intensität mit steigender Temperatur abnimmt $(\Delta I / \Delta T=$ $-0.013 /{ }^{\circ} \mathrm{C}$ ). Sulforhodamin 101 weist dagegen nur eine sehr geringe Temperaturabhängigkeit auf $\left(-0.002 /{ }^{\circ} \mathrm{C}\right)$.

Das Verhältnis der Fluoreszenzintensitäten beider Farbstoffe (Rhodamin B und Sulforhodamin 101) ist in Abb. 4.5 unten gegen die Temperatur aufgetragen. Dabei wurde wie in Abschnitt 4.3 angegeben jeweils der Mittelwert über einen Bildausschnitt gebildet. Es ergibt sich ein linearer Abfall des Intensitätsverhältnisses bei steigender Temperatur. Aus der linearen Anpassung erhält man eine Temperaturempfindlichkeit von $-0.008 /{ }^{\circ} \mathrm{C}$. Außerdem fällt auf, dass die Fehler im Vergleich zu den einzelnen Farbstoffen (Abb. 4.5 oben) wesentlich kleiner geworden sind.

Aus der Messung mit dem Photodetektor ergibt sich eine Standardabweichung der Anregungspulsenergie von $3 \%$ für die gesamte Kalibrierung. 

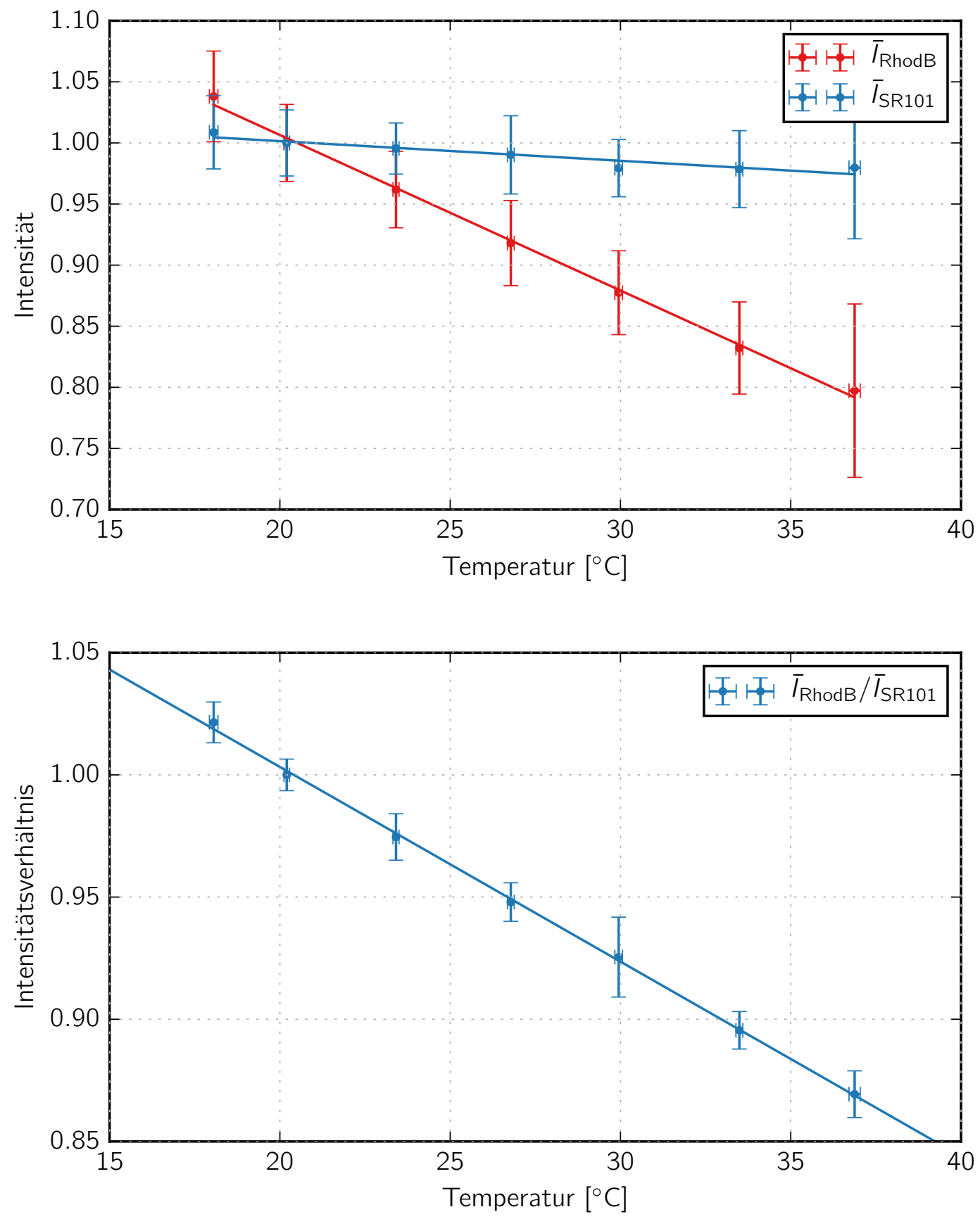

Abbildung 4.5: Gemessene Fluoreszenzintensitäten von Rhodamin B und Sulforhodamin 101 (oben) und Verhältnis der Fluoreszenzintensitäten von Rhodamin B und Sulforhodamin 101 (unten), jeweils bei verschiedenen Wassertemperaturen. In beiden Abbildungen wurden die Intensitäten auf den Wert bei $20^{\circ} \mathrm{C}$ normiert. 


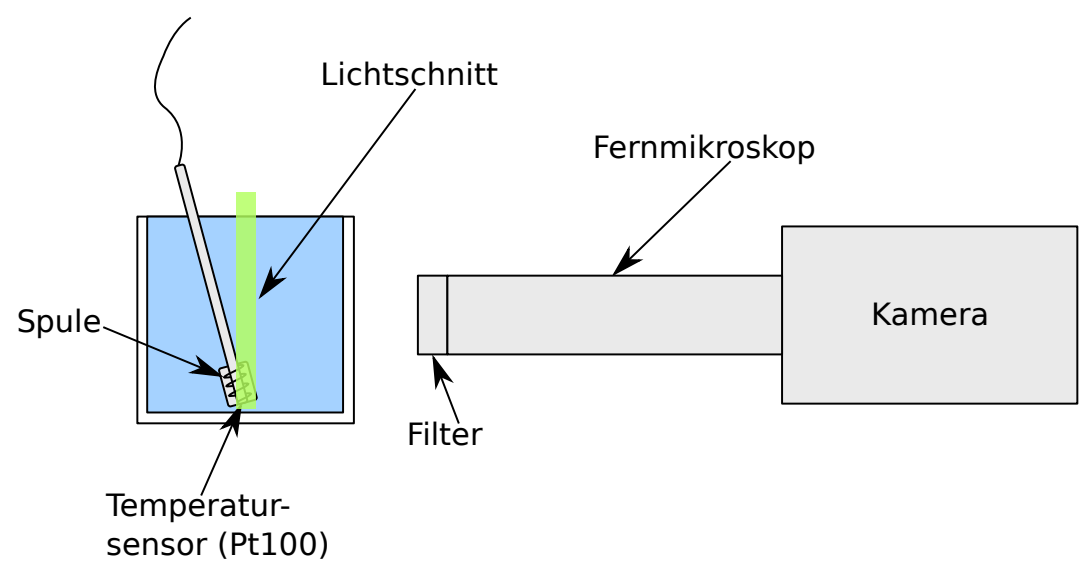

Abbildung 4.6: Testaufbau mit Heizelement in Rhodamin B Lösung.

\subsubsection{Lokales Heizen mit kleiner Spule}

Zum Testen der Temperaturmessung mit der LIF-Methode soll eine Farbstofflösung lokal erwärmt werden. Dazu hat die Elektronikwerkstatt des 3. Physikalischen Instituts ein Heizelement konstruiert, das aus einem Pt100-Sensor mit einer darum gewickelten Spule besteht (Abb.4.6). Fließt ein Strom durch die Spule, so erwärmt sie sich. Im Experiment werden Ströme von 1 bis $2 \mathrm{~A}$ an die Spule angelegt und damit die Farbstofflösung in einer Küvette lokal erwärmt. Einmal pro Sekunde wird die Farbstofflösung (Rhodamin B, $C=1 \cdot 10^{-7} \mathrm{moll}^{-1}$ ) mit einem Laserpuls angeregt und das Fluoreszenzsignal mit einer Kamera aufgenommen. Vor dem Einschalten des Heizelements werden 30 Referenzbilder aufgenommen. Man erwartet bei Erwärmung ein Absinken der Intensität (siehe Abb.4.5).

Aus der Fluoreszenzintensität wird mit Hilfe einer Kalibrierung wie in Abschnitt 4.3 beschrieben die Temperaturänderung bestimmt. Die Ergebnisse für einige ausgewählte Zeitpunkte sind in Abb. 4.7 gezeigt. Das Heizelement ist in den Bildern nicht sichtbar; es befindet sich einige Millimeter unterhalb der unteren Bildkante. Es sind Bereiche erhöhter und verminderter Temperatur erkennbar. In den Rohdaten treten lokal erhöhte Intensitäten auf, die in der Auswertung zu negativen Temperaturänderungen führen. Tatsächlich treten allerdings solche niedrigen Temperaturen in der Küvette nicht auf. 

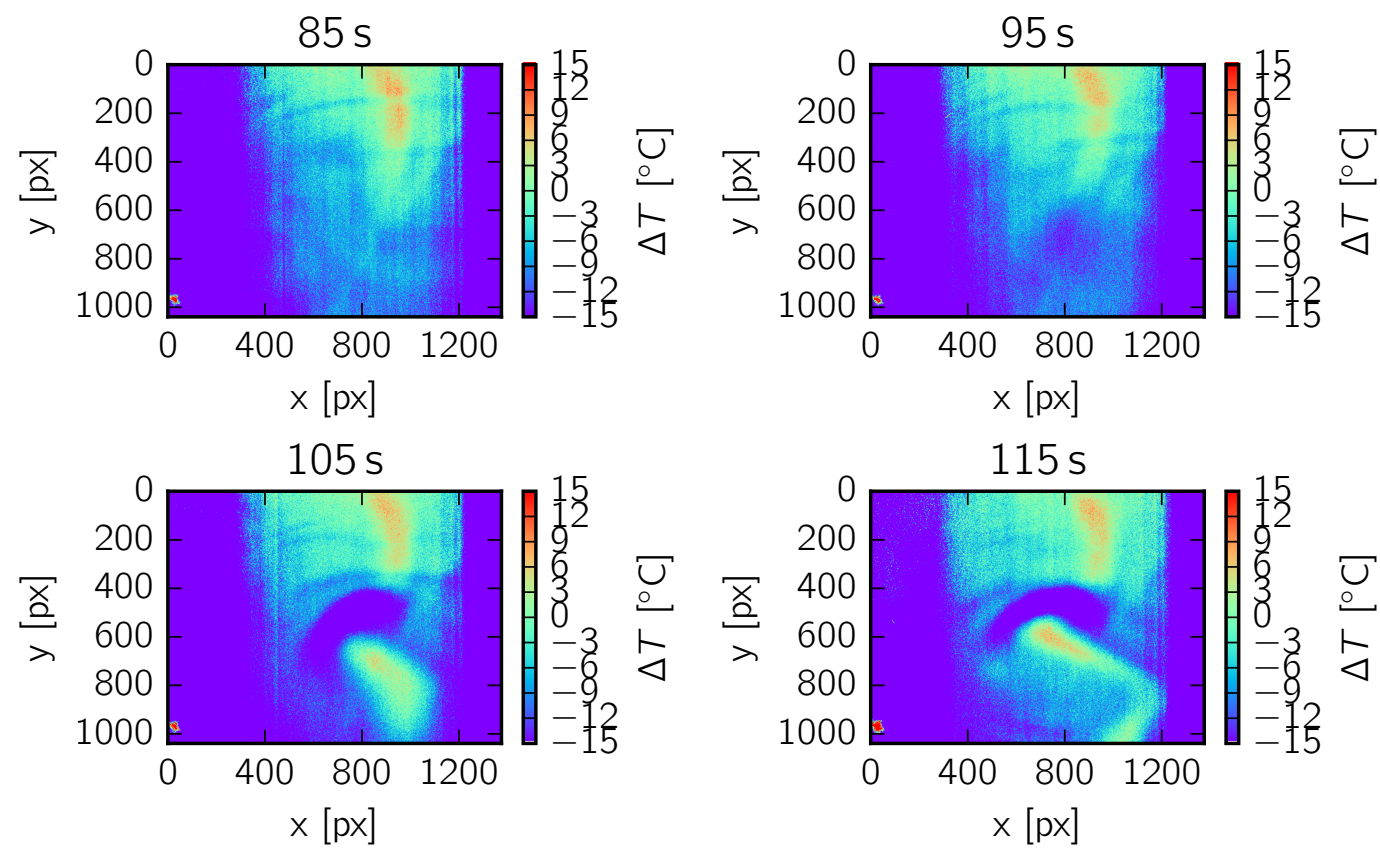

Abbildung 4.7: Ausgewählte Ergebnisse der Testmessung mit Heizelement (unterhalb des Bildausschnitts) in Rhodamin B Lösung. Die Farbe gibt die Abweichung von der Umgebungstemperatur $T=20.0^{\circ} \mathrm{C}$ an. Die Zeitpunkte der Aufnahmen sind jeweils relativ zum Einschalten des Heizelements angegeben.

\subsubsection{Glasküvette mit Peltierelement an einer Seite}

In einem weiteren Testexperiment wurde ein Peltierelement an eine Seitenwand einer Glasküvette geklebt. In der mit Farbstofflösung (Rhodamin B, $C=1 \cdot 10^{-6} \mathrm{moll}^{-1}$ und Sulforhodamin 101, $C=2 \cdot 10^{-6} \mathrm{~mol}^{-1}$ ) gefüllten Küvette befindet sich ein Pt100-Sensor, der ebenso wie das Peltierelement an eine Temperaturregelung angeschlossen ist. Die Anregung der Farbstoff und das Auslösen der Kameras erfolgt zweimal pro Sekunde. Zunächst werden 10 Referenzbilder aufgenommen. Anschließend wird die Solltemperatur auf $30^{\circ} \mathrm{C}$ eingestellt.

Das Ergebnis dieser LIF-Testmessung ist in Abb. 4.8 dargestellt. An der linken Küvettenwand wurde mit dem Peltierelement geheizt. Die mit dem Pt100-Sensor gemessene Temperatur in Wandnähe betrug etwa $30^{\circ} \mathrm{C}$. Es ist deutlich ein Temperaturgefälle von links (warm) nach rechts (kalt) zu erkennen. Zum Vergleich sind in Abb. 4.8 zwei ver- 

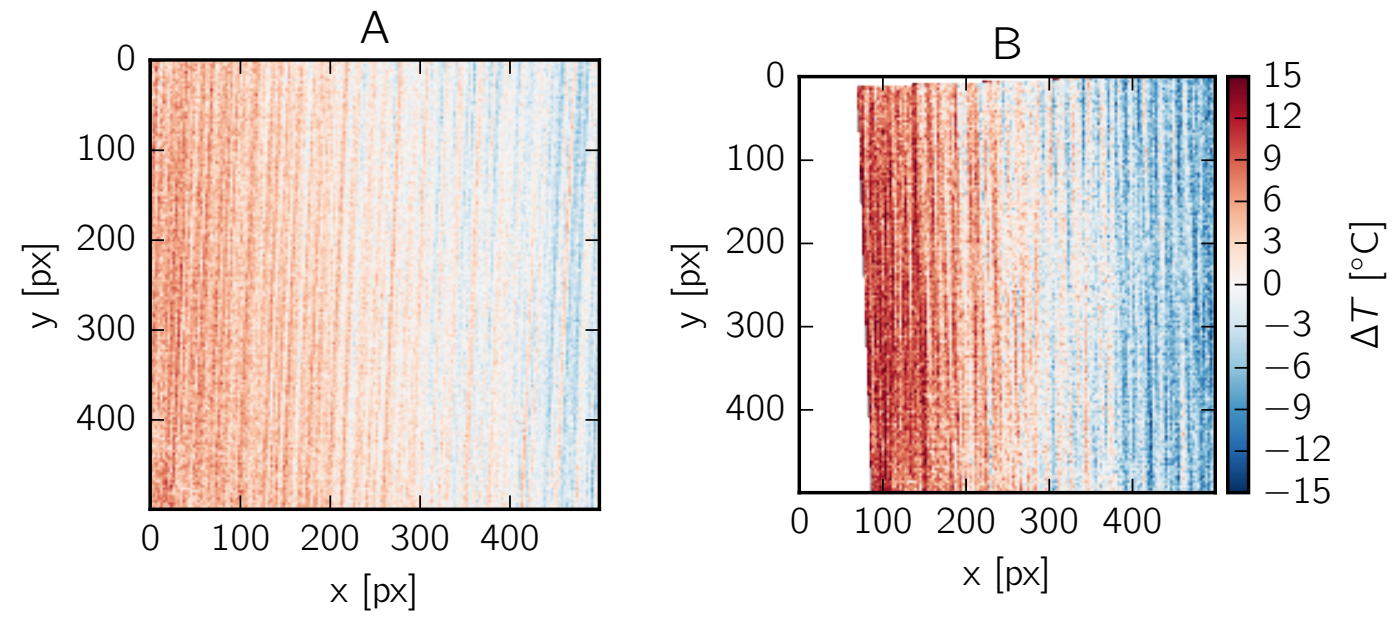

Abbildung 4.8: Ergebnis einer Testmessung, bei der mit einem Peltierelement die linke Küvettenwand erwärmt wird. In der Küvette befindet sich eine Lösung mit beiden Farbstoffen Rhodamin B und Sulforhodamin 101. Auswertung des Kamerabildes nur für Rhodamin B (A); Auswertung beider Kamerabilder für Rhodamin B und Sulforhodamin 101 (B). Im weißen Bereich auf der linken Seite (B) sind nur Pixelwerte von einer Kamera vorhanden, da die Bilder der beiden Kameras gegeneinander verschoben und verdreht sind.

schiedene Ergebnisse der gleichen Messung dargestellt: Links wurde nur das Signal der einen Kamera für Rhodamin B ausgewertet; rechts wurden die Signale beider Kameras (Rhodamin B und Sulforhodamin 101) ausgewertet.

Wie auch in Abschnitt 4.4.4 treten hier in den Rohdaten erhöhte Intensitäten auf, die in der Auswertung zu negativen Temperaturänderungen führen. Die tatsächliche Temperatur in der Küvette betrug aber je nach Position zwischen 20 und $30^{\circ} \mathrm{C}$.

\subsubsection{Abhängigkeit der Fluoreszenzintensität von der Energie des Anregungspulses}

Die vom Farbstoff abgegebene Fluoreszenzintensität ist begrenzt. Bei einer Erhöhung der Anregungsintensität wird also ab einem bestimmten Punkt eine Sättigung eintreten und die Fluoreszenzintensität nicht weiter ansteigen. Bei extrem starker Anregung 


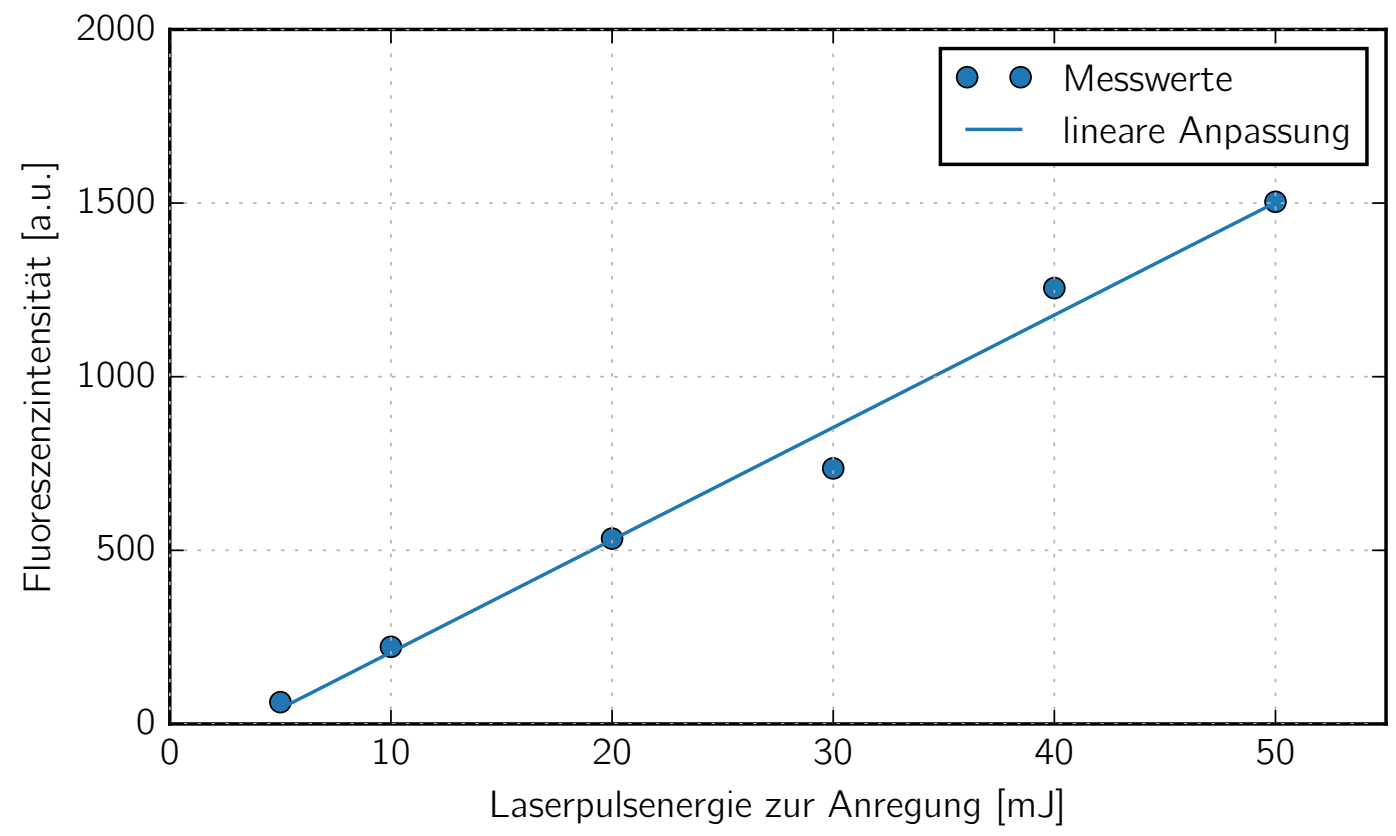

Abbildung 4.9: Abhängigkeit der Fluoreszenzintensität von der Laserpulsenergie zur Anregung.

können sogar die Farbstoffmoleküle zerstört werden, so dass sie nicht mehr fluoreszieren können.

Zur Überprüfung, ob im verwendeten Pulsenergiebereich eine Sättigung auftritt, werden für die Anregung verschiedene Pulsenergien eingestellt und eine Kamera nimmt das Fluoreszenzlicht auf (jeweils 30 Bilder). Die Pulsenergie wird mit einem Laserleistungsmessgerät (Coherent Fieldmaster) bestimmt.

Für jede eingestellte Pulsenergie wird über alle Kamerabilder gemittelt. Eine weitere Mittelung über einen Bildausschnitt liefert einen einzelnen Wert für die Fluoreszenzintensität. Die Ergebnisse sowie eine lineare Anpassung sind in Abb. 4.9 eingetragen. Bei den hier verwendeten Pulsenergien im Bereich von 5 bis $50 \mathrm{~mJ}$ ist keine Sättigung erkennbar. 

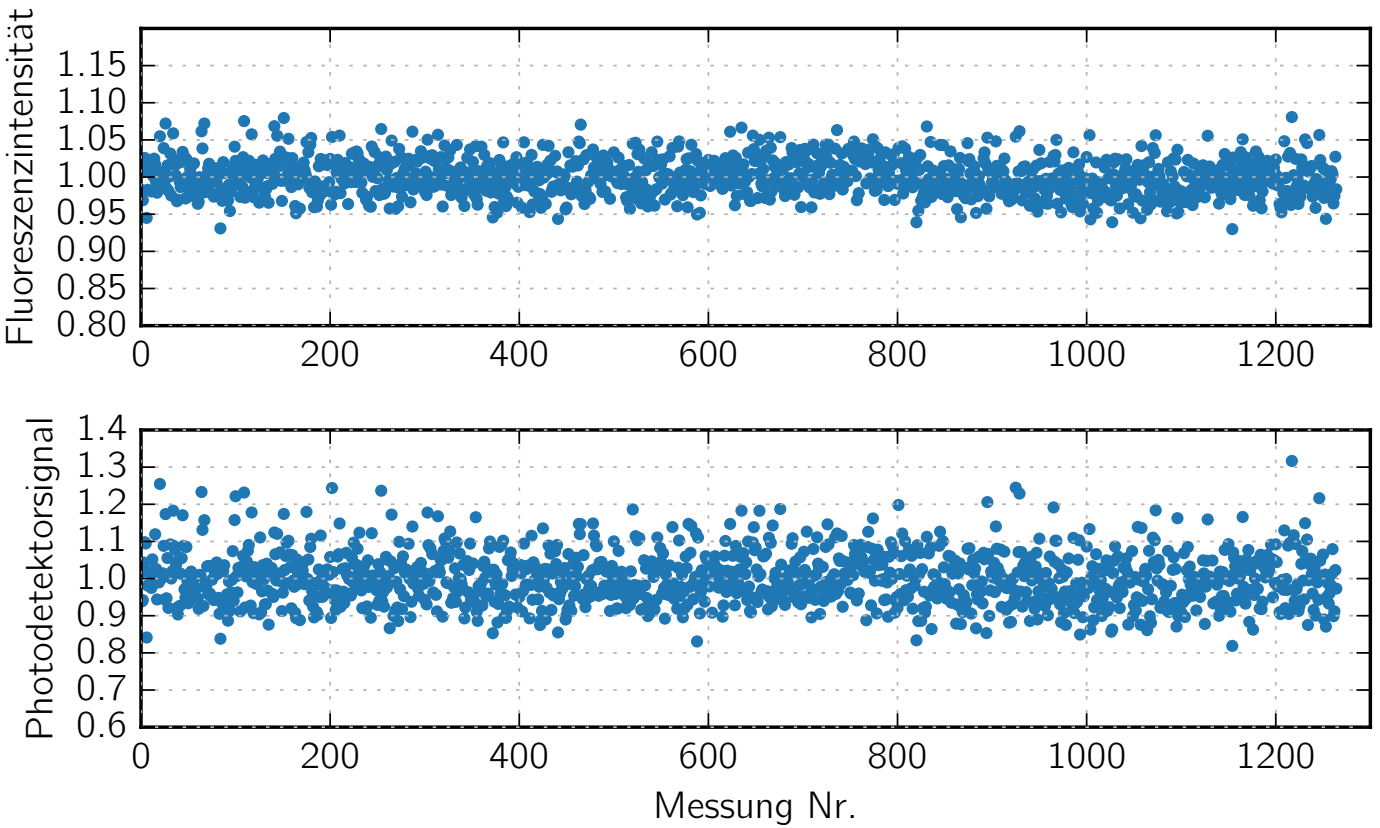

Abbildung 4.10: Langzeitmessung von LIF-Intensität und Photodetektorsignal (Anregungspuls) über einen Zeitraum von $1.75 \mathrm{~h}$. Beide Größen sind auf ihren Mittelwert normiert.

\subsubsection{Ausbleichen der Farbstoffe (Photobleaching)}

Wenn Fluoreszenzfarbstoffe sehr stark oder sehr oft angeregt werden, können sie ausbleichen (photobleaching). Daher wird in diesem Testexperiment eine Farbstofflösung mit Rhodamin B alle $5 \mathrm{~s}$ unter den gleichen Bedingungen wie bei der Temperaturmessung angeregt und das Fluoreszenzlicht mit der Kamera aufgenommen. Eine Mittelung über alle Pixel ergibt für jedes aufgenommene Bild einen Intensitätswert. Ein Photodetektor überwacht die Energie der Anregungspulse.

Bei der in Abb. 4.10 dargestellten Langzeitmessung ist auch nach $1.75 \mathrm{~h}$ und über 1200 Laserschüssen kein Ausbleichen des Farbstoffs erkennbar. 


\subsection{LIF-Messung an laserinduzierter Blase}

Ein gepulster Laser $(\lambda=1064 \mathrm{~nm})$ erzeugt wie in Abschnitt 4.2 beschrieben eine einzelne Blase und es wird eine LIF-Temperaturmessung vorgenommen. Von jeder laserinduzierten Blase nehmen beide Kameras jeweils ein Bild auf. Die Verzögerungszeit zwischen Blasenerzeugung und Belichtung wird variiert, so dass man Momentaufnahmen $\mathrm{zu}$ verschiedenen Zeitpunkten der Blasenschwingung bekommt. Bei jeder Messung erzeugt ein Laserpuls eine einzelne Blase, die nach dem ersten Aufschwingen wenige Male kollabiert.

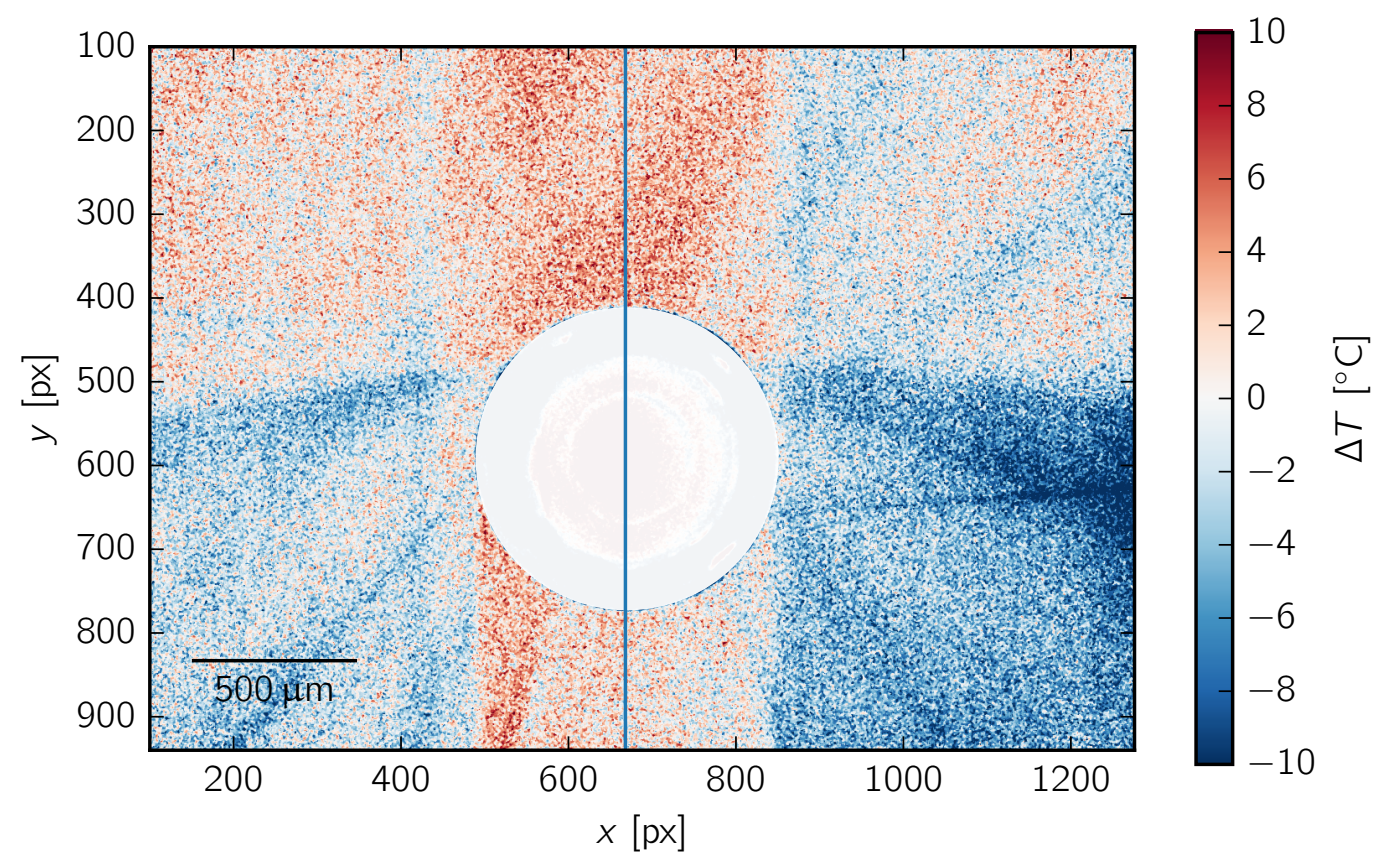

Abbildung 4.11: LIF-Aufnahme einer kollabierenden Laserblase; Kollapszeit: $78 \mu \mathrm{s}$; aufgenommen $11.0 \mu$ s vor dem ersten Kollaps. Die Farbe gibt die gemessene Abweichung $\Delta T$ von der Umgebungstemperatur $T=20.6{ }^{\circ} \mathrm{C}$ an.

Abbildung 4.11 zeigt ein Ergebnis der LIF-Messungen an einer laserinduzierten Blase mit einem maximalen Radius von $R_{\max }=459 \mu \mathrm{m}$. Die Aufnahme wurde $11 \mu$ s vor dem ersten Kollaps gemacht. Dargestellt ist die Temperaturänderung bezüglich der Umgebungstemperatur $T=20.6^{\circ} \mathrm{C}$ in der Küvette. An der Blasenwand wird das Fluoreszenzlicht reflektiert und gebrochen, so dass es zu Artefakten im LIF-Bild kommt. Nur im Bereich oberhalb der Blase $(100<y<411)$ erhält man aussagekräftige Temperatur- 


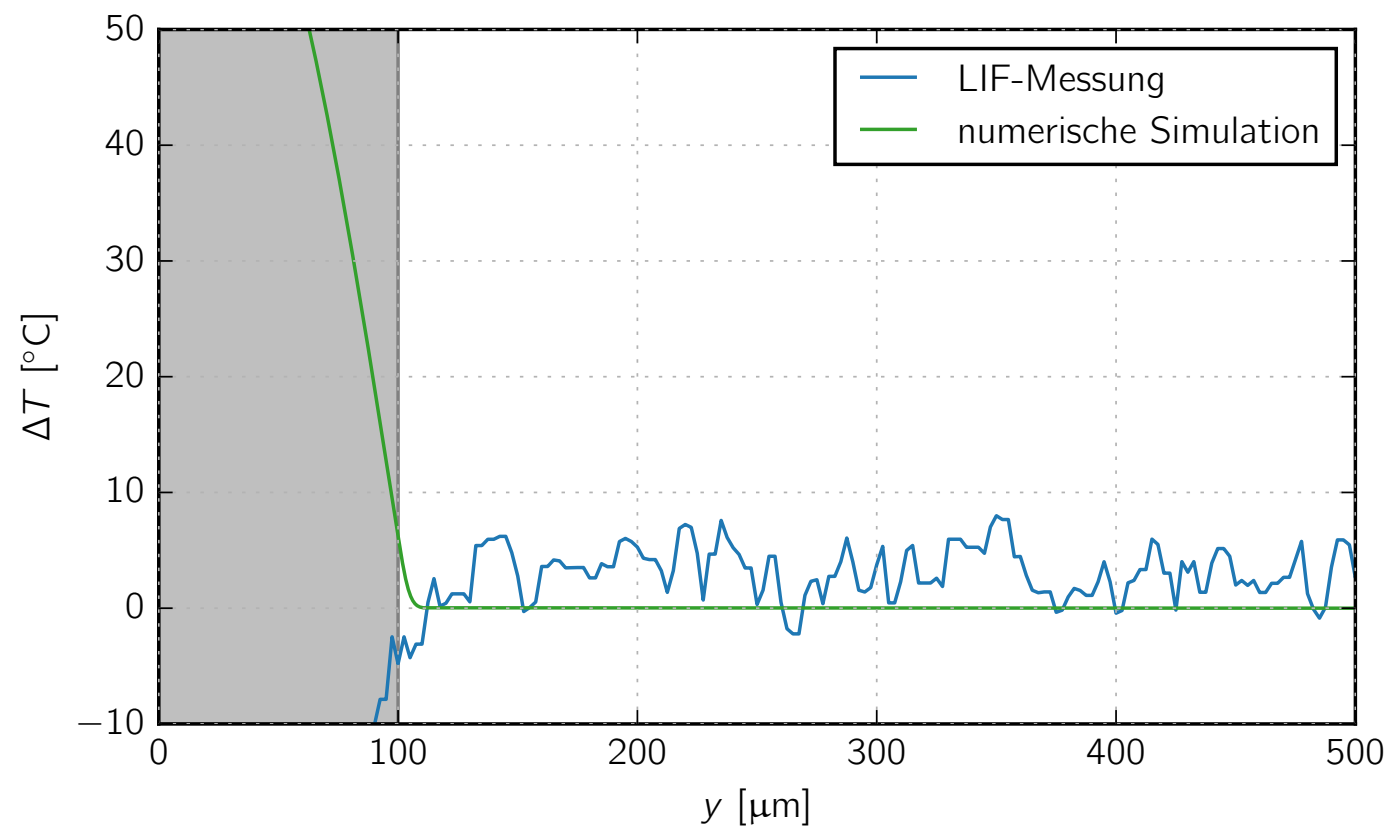

Abbildung 4.12: Schnitt durch die Temperaturverteilung in Abb. 4.11 entlang der Geraden $x=668$. Die Blase ist grau dargestellt. Die Simulation wurde wie in Abschnitt 2.3 beschrieben durchgeführt (Blasenradius $R=100 \mu \mathrm{m}$, Anfangstemperatur in der Blase $T=100^{\circ} \mathrm{C}$ ).

werte. Da innerhalb der Blase keine Temperaturmessung möglich ist, wurde die Blase ausmaskiert. Die Abb. 4.12 zeigt einen Schnitt durch die Blase in Abb. 4.11 entlang der Geraden $x=668$. Der graue Bereich stellt die Blase dar.

In der räumlichen Temperaturverteilung treten Fluktuationen in der Größenordnung von $\pm 8^{\circ} \mathrm{C}$ auf. Der erwartete Temperaturanstieg in der Flüssigkeit nah an der Blase ist nicht zu erkennen.

Die vertikalen Streifen in den LIF-Aufnahmen entstehen durch den inhomogenen Lichtschnitt, sind also durch das inhomogene Strahlprofil des Lasers begründet. Außerdem sind in den Aufnahmen horizontale Streifen zu erkennen, die durch das zeilenweise Auslesen des CCD-Chips in der Kamera zustande kommen. Der Lichtschnitt kann die Blase nicht ungehindert passieren. Es tritt Brechung und Reflexion an der Blasenwand auf. 

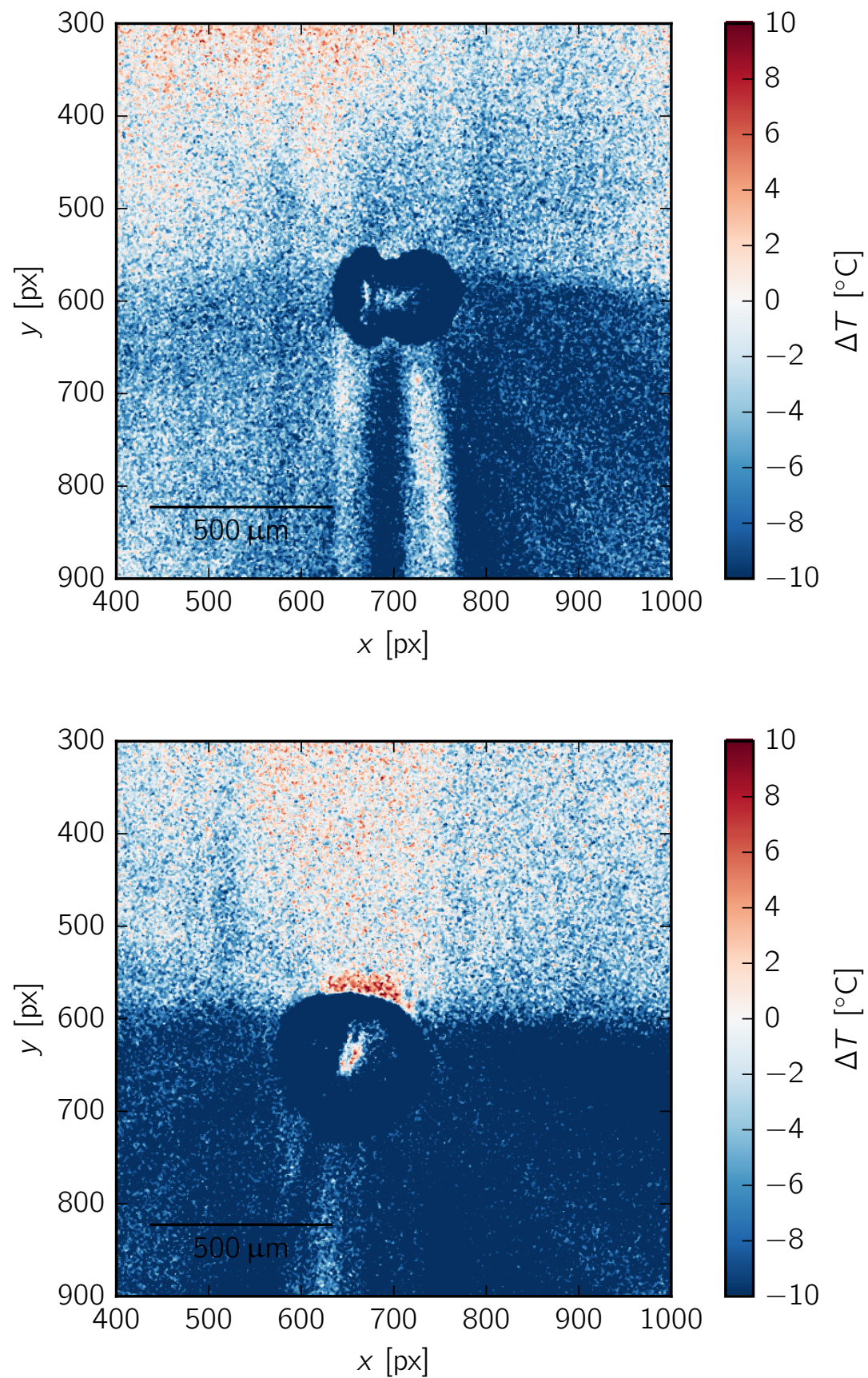

Abbildung 4.13: LIF-Aufnahme einer kollabierenden Laserblase. Kollapszeit: 78 s; aufgenommen $1.4 \mu$ s vor dem ersten Kollaps (oben). Kollapszeit: $76 \mu \mathrm{s}$; aufgenommen $23.1 \mu$ s nach dem ersten Kollaps (unten). 
Ein Pixel im Kamerabild entspricht $2.5 \mu \mathrm{m}$, die erwartete Grenzschichtdicke (siehe Anfang dieses Kapitels) beträgt also etwa 1 px. Die Temperaturverteilung innerhalb der Grenzschicht kann damit nicht aufgelöst werden.

In Abb. 4.13 sind noch zwei weitere Ergebnisse der LIF-Messungen an einer lasererzeugten Blase dargestellt. Die Messung im oberen Bild wurde 1.4 $\mu$ s vor dem ersten Kollaps gemacht; hier ist in der Nähe der Blase keine Temperaturänderung zu erkennen. Das untere Bild in Abb. 4.13 zeigt eine Blase $23.1 \mu$ s nach ihrem ersten Kollaps. In diesem Fall beobachtet man oberhalb der Blase einen Temperaturanstieg von $6{ }^{\circ} \mathrm{C}$.

In den Ergebnissen zeigen sich starke Schwankungen der gemessenen Temperatur. Ein möglicher Grund dafür ist das Rauschen der CCD-Kameras, denn das Fluoreszenzsignal ist relativ schwach. Eine Mittelung über mehrere Aufnahmen ist in diesem Experiment nicht sinnvoll, da die Reproduzierbarkeit der lasererzeugten Blasen dafür nicht ausreicht. Jede Blase ist individuell und insbesondere das Kollapsverhalten kann sich von Blase zu Blase stark unterscheiden.

In den LIF-Ergebnissen ist nur ein kleiner Temperaturanstieg in der Nähe der Blase erkennbar. Dies liegt wohl einerseits daran, dass die Auflösung der verwendeten Abbildungsoptik nicht ausreicht, um die thermische Grenzschicht aufzulösen. Möglicherweise wird keine Wärme oder nur eine so geringe Wärmemenge von der Blase an die Flüssigkeit abgegeben, dass in diesem Experiment der Temperaturanstieg kaum messbar ist. Außerdem kann Wärmetransport vom Blaseninneren zur Flüssigkeit nur im Kollaps stattfinden, also in einem sehr kleinen Zeitfenster von wenigen Mikrosekunden. Dazu kommt noch, dass die Wärmeleitfähigkeit von Wasser relativ hoch ist und daher ein eventueller Temperaturgradient im Wasser schnell wieder verschwindet. Das Volumen der kollabierenden Blase und damit das Volumen des erwärmten Wassers sind im Vergleich mit dem gesamten Wasservolumen in der Küvette sehr klein.

\subsection{LIF-Messung an einer Blase im Schallfeld}

Die LIF-Messung an einer einzelnen im Schallfeld gefangenen Blase erfolgt ähnlich wie die im vorigen Abschnitt beschriebene Messung an einer lasererzeugten Blase. Als Blasenfalle wird eine mit der Farbstofflösung (Rhodamin B, $C=1 \cdot 10^{-6} \mathrm{moll}^{-1}$ ) gefüllte Glasküvette verwendet, auf deren Unterseite eine Piezoscheibe geklebt ist. Vor der Messung wird die Farbstofflösung ca. 15 min mit einer Vakuumpumpe entgast. Dann wird die Lösung in die Küvette gefüllt und ein Frequenzgenerator mit nachgeschaltetem Verstärker erzeugt darin ein Ultraschallfeld mit einer Frequenz von etwa $24 \mathrm{kHz}$. Mit Hilfe 
einer Spritze wird etwas Luft in die Lösung gebracht und somit eine Blase produziert, die sich anschließend in die Mitte der Küvette bewegt und dort im Schallfeld oszilliert. Mit bloßem Auge kann man erkennen, dass die Blase leuchtet - es tritt Sonolumineszenz auf.

Die beiden Kameras nehmen unabhängig von der Frequenz des Schallfeldes jeweils 1 Bild pro Sekunde auf, so dass man Bilder zu verschiedenen Phasen der Blasenschwingung erhält. Die Belichtungszeit beträgt $10 \mu \mathrm{s}$.

Auch hier wird eine LIF-Messung an einer einzelnen Blase durchgeführt. Im Unterschied zur lasererzeugten Blase führt die Blase im Schallfeld aber viele Schwingungen und damit viele Kollapse aus. Über einen längeren Zeitraum betrachtet, müsste also eine größere Wärmemenge an das Wasser abgegeben werden als im Fall der frei schwingenden Laserblase.
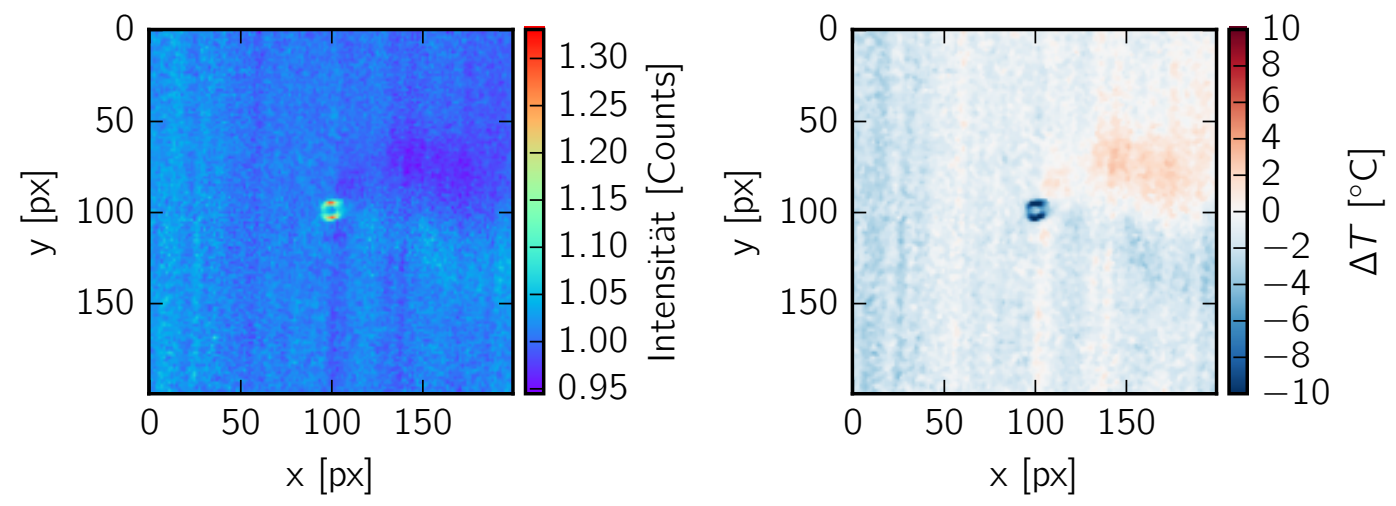

Abbildung 4.14: Ergebnis einer LIF-Messung an einer Blase in Rhodamin B Lösung $\left(C=1 \cdot 10^{-6} \mathrm{~mol}^{-1}\right)$, die durch ein Schallfeld mit einer Frequenz von $24 \mathrm{kHz}$ angeregt wird. Gemessene Fluoreszenzintensität (links) und aus der Intensität berechnete Temperaturabweichung $\Delta T$ von der Umgebungstemperatur $T=21^{\circ} \mathrm{C}$ (rechts). 
In Abb. 4.14 ist ein Ergebnis der LIF-Messungen an einer einzelnen im Schallfeld gefangenen Blase dargestellt, das wie in Abschnitt 4.3 beschrieben aus den aufgenommenen Kamerabildern gewonnen wurde.

Bei ausreichend starker Anregung der Blase, d.h. bei ausreichend hoher Schalldruckamplitude, ist ein Bereich niedrigerer Fluoreszenzintensität sichtbar („dunkle Wolke“). Dies lässt sich auch beobachten, wenn sich nur Sulforhodamin 101 (nicht temperaturabhängig) in der Lösung befindet. Daher kommt die niedrigere Fluoreszenzintensität vermutlich nicht durch eine Temperaturänderung zustande. Eine mögliche Ursache für die verminderte Fluoreszenzintensität in diesem LIF-Experiment ist Fluoreszenzlöschung durch OH-Radikale. Letztere können beim heftigen Kollaps einer im Schallfeld angeregten Blase entstehen [51].

Das LIF-Ergebnis in Abb. 4.14 sieht ähnlich aus, wie ein von Verraes et al. veröffentlichtes Bild von einer schwingenden Blase in einer $\mathrm{CS}_{2}$ Lösung [53].

\subsection{Ausblick}

Um die schnelle Blasenschwingung und insbesondere den Blasenkollaps zeitlich besser auflösen zu können, bietet es sich an, die LIF-Messungen mit einer Hochgeschwindigkeitskamera durchzuführen. Die Farbstoffe müssten dazu kontinuierlich oder mit der Wiederholrate der Kamera angeregt werden. Zusätzlich ist eine höhere Vergrößerung wünschenswert, um die thermische Grenzschicht räumlich auflösen zu können. Dann sinkt aber die Intensität des in die Kamera einfallenden Fluoreszenzlichts noch weiter. Eine Möglichkeit wäre, den Anregungslaserpuls stärker zu fokussieren, also das Lichtschnittvolumen zu verkleinern. Dabei muss man allerdings beachten, dass die Farbstoffmoleküle bei hohen Leistungsdichten zerstört werden können.

In den hier vorgestellten Experimenten wurden kugelförmige Blasen mit der LIF-Methode untersucht. Interessant wäre auch eine LIF-Temperaturmessung an asphärisch kollabierenden Blasen. Wenn am Anfang der Messung ein Temperaturgradient in der Küvette eingestellt wird (wie in Abschnitt oder in [63]), dann sollte eine Vermischung von warmen und kalten Flüssigkeitsanteilen sichtbar werden, die durch die Jetströmung der kollabierenden Blase hervorgerufen wird. Möglich wäre damit auch eine Messung der Jetgeschwindigkeit.

Die Messung an einer einzelnen Blase im Schallfeld deutet darauf hin, dass die LIFMethode dazu verwendet werden könnte, chemische Reaktionen bzw. deren Produkte 
sichtbar zu machen.

Anstatt organischer Farbstoffe wie Rhodamin B kann man auch thermochromische Flüssigkristalle zur Temperaturmessung verwenden [70]. Dazu ist allerdings eine Farbkamera notwendig.

Bisher ist noch nicht geklärt, welche Temperaturen innerhalb der Blase erreicht werden. Eine Messung der Temperatur im Innern der Blase ist mit laserinduzierter Fluoreszenz nicht möglich, da die Farbstoffe nur in der Flüssigkeit gelöst sind. Die Temperatur in der Blase kann indirekt bestimmt werden durch Spektroskopie an leuchtenden Einzelblasen (SBSL) [71] oder Vielblasensystemen (MBSL) [72, 73]. 


\section{Zusammenfassung und Ausblick}

\subsection{Zusammenfassung}

In dieser Arbeit wird der Einfluss der Wassertemperatur auf die Dynamik von einzelnen Kavitationsblasen sowie der Wärmetransport während der Blasenschwingung untersucht.

In den Experimenten mit einzelnen lasererzeugten Blasen in Wasser bei erhöhten Temperaturen bis $80^{\circ} \mathrm{C}$ hat sich gezeigt, dass der Dampfdruck einen wesentlichen Einfluss auf die Blasendynamik hat. Bei hohen Wassertemperaturen kollabiert eine Blase langsamer und ihr Radius wird aufgrund des höheren Dampfgehalts im Kollaps nicht so klein wie bei niedrigen Temperaturen. Dies könnte für die Kavitationserosion relevant sein.

Zum Vergleich mit den Messdaten wurden numerische Rechnungen mit verschiedenen Modellen für sphärische Blasen durchgeführt. Die Ergebnisse zeigen, dass Phasenübergänge während der Blasenschwingung eine wichtige Rolle spielen. Die Dämpfung einer Blasenschwingung wird neben Schallabstrahlung, viskoser Reibung und Wärmeleitung offenbar auch durch Phasenübergänge beeinflusst.

Bei den Temperaturmessungen mittels laserinduzierter Fluoreszenz an einer einzelnen Blase im Schallfeld ist ein deutliches Signal erkennbar. Allerdings ist noch unklar, ob es sich dabei um eine Temperaturerhöhung handelt oder ob Fluoreszenzlöschung durch sonochemische Reaktionsprodukte auftritt. In den Ergebnissen der LIF-Messungen an einer einzelnen lasererzeugten Blase ist kaum eine Erwärmung des Wassers erkennbar. Allerdings liegt die erwartete Dicke der thermischen Grenzschicht um die Blase an der optischen Auflösungsgrenze des verwendeten Aufbaus. Wenn beim Kollaps einer lasererzeugten Blase Wärmetransport stattfindet, dann muss die von der Blase an das Wasser abgegebene Wärmemenge sehr klein sein.

Es wurde eine Methode zur Visualisierung von Stoßwellen vorgestellt und ausgewählte Ergebnisse zur Stoßwellenausbreitung beim sphärischen sowie beim asphärischen 
Blasenkollaps gezeigt. Dabei kann man auch sogenannte Kopfwellen beobachten, die dadurch entstehen, dass die Stoßwelle, sobald sie auf eine feste Wand trifft, sich auch innerhalb dieser Wand ausbreitet.

\subsection{Ausblick}

Im Anschluss an das Experiment mit frei schwingenden lasererzeugten Blasen könnte man den asphärischen Kollaps vor einer festen Wand bei verschiedenen Wassertemperaturen untersuchen. Dabei ist auch der Einfluss der Wassertemperatur auf die Kavitationserosion von Interesse.

Wenn man das Toegel Modell auf dem Gilmore Modell aufbaut (anstatt Keller-Miksis), könnte dies eine Verbesserung bringen, da das Gilmore Modell laut [15] dem KellerMiksis Modell überlegen ist. Die Modellierung der Blasendynamik ließe sich verbessern, wenn man die vollständigen strömungsmechanischen Gleichungen verwendet und dabei auch die Temperatur berücksichtigt. Dazu könnte man von dem Modell in [18] ausgehen und zusätzlich eine Energiegleichung einführen. Darüber hinaus wäre die Berücksichtigung von Phasenübergängen im Modell wünschenswert, was allerdings einen hohen Aufwand bedeutet.

Um in der LIF-Temperaturmessung die thermische Grenzschicht an der Blasenwand räumlich auflösen zu können, ist eine höhere Vergrößerung nötig. Als Alternative zu organischen Farbstoffen könnte man thermochromische Flüssigkristalle für eine Temperaturmessung verwenden [70], braucht dazu allerdings eine Farbkamera. Desweiteren kann die LIF-Methode möglicherweise eingesetzt werden, um sonochemische Reaktionsprodukte sichtbar zu machen.

Sämtliche Stoßwellenaufnahmen in dieser Arbeit wurden bei Raumtemperatur gemacht. Interessant wäre es, den Einfluss der Wassertemperatur auf den maximalen Schalldruck der abgestrahlten Stoßwellen zu untersuchen (z. B. mit einem Faserhydrofon). In diesem Zusammenhang ist, wie oben bereits erwähnt, der Einfluss der Wassertemperatur auf die Kavitationserosion interessant und von praktischer Bedeutung. 
Anhang 



\section{A Stoßwellen beim Blasenkollaps vor einer festen Wand}

Bei lasererzeugten Blasen werden Stoßwellen im Laserdurchbruch und im Kollaps abgestrahlt. Stoßwellen breiten sich in Form von Druckänderungen in einem Medium aus. Im vorliegenden Fall laufen also Druckänderungen durch das Wasser in einer Küvette. Die Druckänderungen gehen mit Dichteänderungen im Wasser einher. Diese Dichteänderungen können im Experiment mit Hilfe des Schattenverfahrens oder des Schlierenverfahrens sichtbar gemacht werden [74]. Die folgenden Abschnitte beschreiben jeweils ein Experiment zur Visualisierung der Stoßwellen - zunächst mit dem Schlierenverfahren, anschließend mit dem Schattenverfahren ähnlich wie in [75].

\section{A.1 Stoßwellenaufnahmen im Schlierenverfahren}

Wie in Abschnitt 3.1 beschrieben wird eine einzelne Blase mit einem gepulsten Laser (Quantel Brio, $\lambda=532 \mathrm{~nm}$, Pulsdauer: $4 \mathrm{~ns}$ ) in einer wassergefüllten Küvette erzeugt. Zur Beleuchtung wird ein LED-Blitz mit einer Pulsdauer von 20 ns verwendet. Ein $4 f$ Aufbau mit Rasierklinge als Filter dient als Schlierenoptik. Die so entstehenden Stoßwellenbilder werden mit einer CCD-Kamera (PCO SensiCam qe) aufgenommen, wobei die Verzögerung zwischen Blasenerzeugung und Auslösen der Kamera mit einem Delaygenerator (Stanford Research Systems DG535) eingestellt werden kann.

Abbildung A.1 zeigt exemplarisch eine Schlierenaufnahme von einer lasererzeugten Blase vor einer Aluminiumwand. Der normierte Abstand Blase-Wand beträgt $\gamma=1.0$. Es sind eine reflektierte Stoßwelle sowie Kopfwellen zu sehen, die durch die Ausbreitung der Stoßwelle innerhalb der Aluminiumwand zustande kommen. 




Abbildung A.1: Asphärischer Kollaps einer Laserblase vor einer Aluminiumwand, aufgenommen mit dem Schlierenverfahren $1.5 \mu$ s nach dem ersten Kollaps. Normierter Wandabstand $\gamma=1.0$. Es sind reflektierte Stoßwellen und Kopfwellen zu erkennen.

\section{A.2 Stoßwellenaufnahmen im Schattenverfahren}

Der experimentelle Aufbau zur Aufnahme von Stoßwellenbildern mit dem Schattenverfahren ist in Abb. A.2 dargestellt und lehnt sich an den in [75] verwendeten Aufbau an. Die Blasen werden mit einem Laser (Spectra Physics Quanta Ray PIV-400, $\lambda=532 \mathrm{~nm}$, Pulsdauer: $8 \mathrm{~ns}$ ) wie in Abschnitt 3.1 beschrieben erzeugt (Laserpuls 1). Laserpuls 2 regt eine Farbstofflösung (Pyridin $1^{1}$ in Ethanol, $C=0.42 \mathrm{~g}^{-1}$ ) an und das Fluoreszenzlicht wird in eine Faser eingekoppelt, an deren Ende sich ein Kollimator befindet. Auf diese Weise erhält man paralleles Licht mit hoher Intensität und kurzer Pulsdauer (etwa $10 \mathrm{~ns})$. Da das Licht inkohärent ist, treten keine störenden Interferenzeffekte auf.

Es wird von jeder mit dem Laser erzeugten Blase ein Bild mit der Kamera (PCO SensiCam qe) aufgenommen. Die Verzögerungszeit zwischen Blasenerzeugung und Auslösen der Kamera kann mit einem Delaygenerator (Stanford Research Systems DG535) präzise eingestellt werden. Da sich Stoßwellen im Normalfall mit Schallgeschwindigkeit ausbreiten, ist für eine Momentaufnahme mit einer Kamera eine sehr kurze Belichtungszeit notwendig. Die Belichtungszeit ist in diesem Fall gegeben durch die Pulsdauer des

\footnotetext{
${ }^{1}$ alternative Bezeichnung: LDS 698
} 


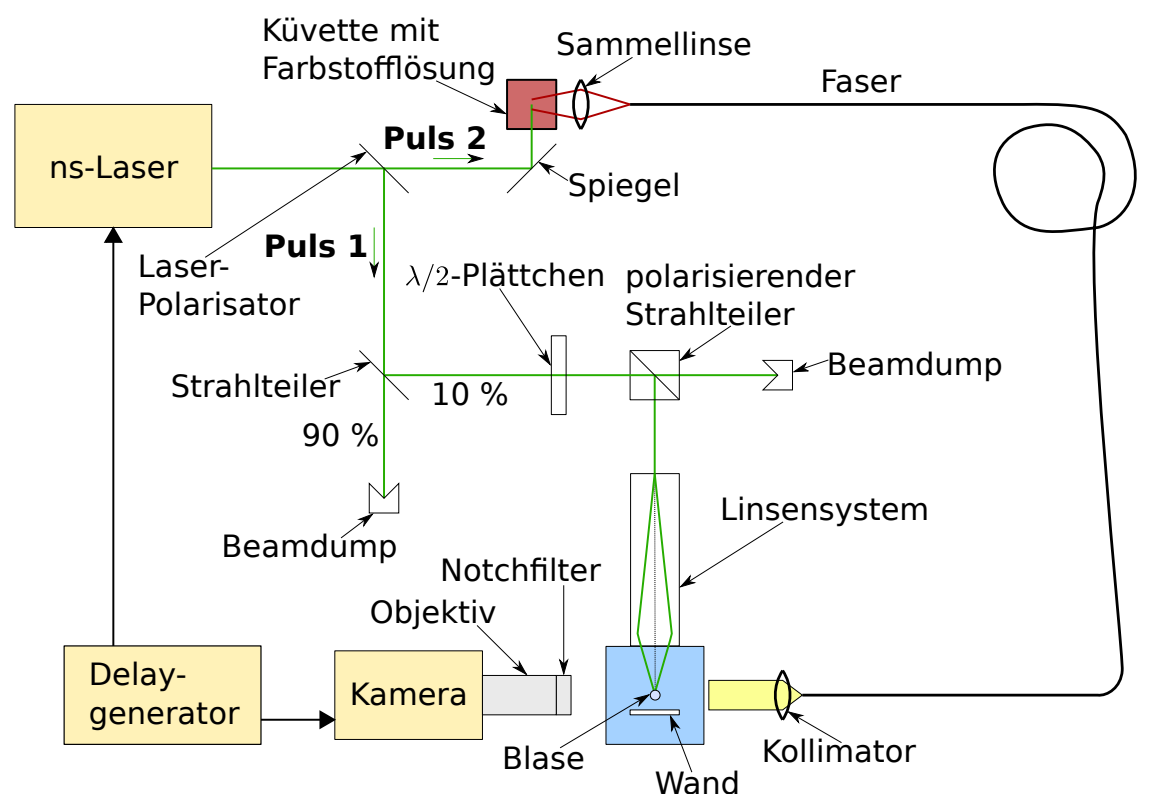

Abbildung A.2: Aufbau zur Aufnahme von Stoßwellenbildern mit dem Schattenverfahren. Laserpuls 1 erzeugt eine Blase in der wassergefüllten Küvette, Laserpuls 2 regt zur Beleuchtung einen Farbstoff an. Das Fluoreszenzlicht wird in eine Faser eingekoppelt und an deren Ende kollimiert.

Lasers sowie die Dauer des Fluoreszenzleuchtens und beträgt etwa 20 ns.

Es werden feste Wände aus verschiedenen Materialien (Stahl, Aluminium, PE-HD) in der Nähe der Blase positioniert, um den asphärischen Kollaps und die Stoßwellenausbreitung zu untersuchen. Der Abstand $d$ des Blasenmittelpunkts von der Wandoberfläche kann variiert werden, so dass man Messungen bei verschiedenen normierten Wandabständen $\gamma=d / R_{\max }$ durchführen kann.

Einige ausgewählte Stoßwellenbilder, die im Schattenverfahren aufgenommen wurden, sind in Abb. A.3 dargestellt. Gegenüber den Schlierenaufnahmen mit dem LED-Blitz in Abschnitt A.1 erkennt man hier auch schwache Stoßwellen wie z. B. Reflexionen an der Küvettenwand (Abb. A.3 oben links). 




Abbildung A.3: Stoßwellen beim asphärischen Blasenkollaps vor einer Stahlwand (rechts im Bild); aufgenommen im Schattenverfahren. Normierter Wandabstand $\gamma=2.4$. Der Zeitpunkt der Aufnahme ist jeweils relativ zum Laserdurchbruch $\left(t_{0}\right)$ angegeben. Die Blase kollabiert bei $t_{0}=$ $148.5 \mu \mathrm{s}$.

\section{A.3 Ausblick}

Die hier gezeigten Stoßwellenbilder wurden bei Raumtemperatur aufgenommen. Im nächsten Schritt könnte man ergänzend zum Experiment in Kap. 3 die Stoßwellenabstrahlung im Blasenkollaps bei verschiedenen Wassertemperaturen beobachten. Ein Hydrofon ermöglicht es, den Einfluss der Temperatur auf den maximalen Druck zu untersuchen. Wegen der extrem hohen Drücke in der Größenordnung von GPa ist ein faseroptisches Hydrofon für dieses Experiment besonders geeignet. Damit lässt sich der Druckverlauf einer Stoßwelle zeitlich aufgelöst messen.

Für ein besseres Verständnis der Kavitationserosion wäre eine ortsaufgelöste Druckmes- 
sung an der Oberfläche der festen Wand, beispielsweise piezoelektrisch oder interferometrisch, hilfreich.

Da es immer kleine Schwankungen in der Pulsenergie des Lasers für die Blasenerzeugung gibt, ist eine stroboskopische Aufnahme der Stoßwellen wie in den hier vorgestellten Experimenten schwierig. Der Zeitpunkt des Blasenkollaps kann sich von Puls zu Puls um etwa $1 \mu$ s verschieben. Mit einer Hochgeschwindigkeitskamera wie z. B. der Hadland Imacon (Wiederholrate bis zu 100 Millionen Bilder pro Sekunde) ist die Aufnahme von mehreren aufeinanderfolgenden Bildern der gleichen Stoßwelle möglich. Die genaue Kollapszeit muss in jedem Fall mit einem Hydrofon bestimmt werden.

Numerische Rechnungen zur Stoßwellenausbreitung beim asphärischen Blasenkollaps wurden u. a. von Bachmann und Müller durchgeführt [76, 77]. Ein Vergleich mit experimentellen Daten kann einerseits dazu dienen, die numerischen Modelle zu verifizieren. Andererseits kann man in den numerischen Simulationen auch experimentell nicht oder nur schwer zugängliche Situationen untersuchen. In der Simulation lassen sich beispielsweise auch Stoßwellen innerhalb der Blase beobachten.

In Abb. A.3 oben rechts sind einige Linien zu erkennen, die etwa sternförmig vom Zentrum der Blase nach außen verlaufen. Hierbei könnte es sich um thermisch bedingte Dichteänderungen in der Flüssigkeit handeln, die durch das Schattenverfahren sichtbar werden. Etwas ähnliches haben Baghdassarian et al. [78] bei lasererzeugten Blasen in flüssigem Stickstoff bei einer Temperatur von $66 \mathrm{~K}$ beobachtet. 



\section{B Linsensysteme zur Blasenerzeugung mit gepulsten Lasern}

Dieser Abschnitt führt alle verwendeten Linsensysteme und die darin verbauten Linsen auf. Die Linsensysteme wurden im Hinblick auf minimale sphärische Aberrationen optimiert. Für die in dieser Arbeit beschriebenen Experimente wurden zwei verschiedene Küvetten verwendet.

Küvette A kommt für die Messungen bei erhöhten Wassertemperaturen zum Einsatz und besteht aus einer äußeren Küvette aus Makrolon (Polycarbonat) und einer inneren Küvette aus Glas. Das Design der Optik (Tab. B.1) basiert auf einer Optik aus [79].

Küvette $B$ wird für die LIF-Messungen verwendet und ist eine Glasküvette der Firma Hellma (Innenabmessungen: $40 \mathrm{~mm} \times 40 \mathrm{~mm} \times 40 \mathrm{~mm}$ ). Die Optik entspricht im Prinzip der für Küvette A. Allerdings befindet sich hier zwischen letzter Linse und Küvette Luft anstatt Wasser (Tab. B.2). Für diese Küvette wurde von Thomas Kurz eine weitere Optik für eine Laserwellenlänge von $\lambda=1064 \mathrm{~nm}$ entworfen (Tab. B.3.

\begin{tabular}{llc}
\hline Nr. & Linsentyp & Abstand zur nächsten Linse [mm] \\
\hline 1 & 01LPX127 & 133.8 \\
2 & LBF254-200 & 50.0 \\
3 & 01LPK003 & 21.23 \\
4 & 01LPX106 & 0.7 \\
5 & 01LPX106 & 8.4 \\
6 & Meniskuslinse & - \\
\hline
\end{tabular}

Tabelle B.1: Linsensystem für Küvette A, $\lambda=532 \mathrm{~nm}$. 


\begin{tabular}{llc}
\hline Nr. & Linsentyp & Abstand zur nächsten Linse $[\mathrm{mm}]$ \\
\hline 1 & 01LPX127 & 133.8 \\
2 & LBF254-200 & 50.0 \\
3 & 01LPK003 & 21.23 \\
4 & 01LPX106 & 0.7 \\
5 & 01LPX106 & 8.4 \\
6 & Meniskuslinse & - \\
\hline
\end{tabular}

Tabelle B.2: Linsensystem für Küvette B, $\lambda=532 \mathrm{~nm}$.

\begin{tabular}{lcc}
\hline Nr. & Linsentyp & Abstand zur nächsten Linse [mm] \\
\hline 1 & LC1582-C & 90 \\
2 & LA1433-C & 19.17 \\
3 & 01LPK003 & 23.98 \\
4 & LA1131-C & 12.0 \\
5 & LA1131-C & 0.4 \\
6 & LE1234-C & - \\
\hline
\end{tabular}

Tabelle B.3: Linsensystem für Küvette B, $\lambda=1064 \mathrm{~nm}$. 


\section{Konstanten, Materialeigenschaften und Parameter}

An dieser Stelle sind die Konstanten, Materialeigenschaften und Parameter, die in dieser Arbeit verwendet werden, aufgeführt.

\begin{tabular}{lllll}
\hline Größe & Symbol & Wert & Einheit & Quelle \\
\hline Boltzmannkonstante & $k_{\mathrm{B}}$ & $1.38066 \cdot 10^{-23}$ & $\mathrm{~J} \mathrm{~K}^{-1}$ & {$[26]$} \\
Avogadrozahl & $N_{\mathrm{A}}$ & $6.02214 \cdot 10^{23}$ & $\mathrm{~mol}^{-1}$ & {$[26]$} \\
universelle Gaskonstante & $R_{\mathrm{g}}$ & 8.31451 & $\mathrm{~J} \mathrm{~mol}^{-1} \mathrm{~K}^{-1}$ & {$[26]$} \\
\hline
\end{tabular}

Tabelle C.1: Konstanten.

\begin{tabular}{lllll}
\hline Größe & Symbol & Wert & Einheit & Quelle \\
\hline Temperaturleitfähigkeit (Luft, 300 K) & $\alpha$ & $22.4 \cdot 10^{-6}$ & $\mathrm{~m}^{2} \mathrm{~s}^{-1}$ & berechnet nach Gl. $[2.38)$ \\
Temperaturleitfähigkeit (Wasser, $20^{\circ} \mathrm{C}$ ) & $\alpha$ & $0.143 \cdot 10^{-6}$ & $\mathrm{~m}^{2} \mathrm{~s}^{-1}$ & berechnet nach Gl. \\
Polytropenexponent & $\kappa$ & $4 / 3$ & - & {$[41]$} \\
(dynamische) Viskosität & $\mu$ & $1.002 \cdot 10^{-3}$ & $\mathrm{~Pa} \mathrm{~s}$ & {$[18]$} \\
Dichte (Luft, 300 K) & $\varrho$ & 1.161 & $\mathrm{~kg} \mathrm{~m}^{-3}$ & {$[34]$} \\
Dichte (Wasser) & $\varrho$ & 998.21 & $\mathrm{~kg} \mathrm{~m}^{-3}$ & {$[18]$} \\
Oberflächenspannung (Wasser) & $\sigma$ & $72.75 \cdot 10^{-3}$ & $\mathrm{~N} \mathrm{~m}^{-1}$ & {$[35]$} \\
Tait Parameter & $B$ & $3046 \cdot 10^{5}$ & $\mathrm{~Pa}^{-1}$ & {$[18]$} \\
Schallgeschwindigkeit (Wasser, $\left.20^{\circ} \mathrm{C}\right)$ & $c_{0}$ & 1483 & $\mathrm{~m} \mathrm{~s}^{-1}$ & {$[80]$} \\
spezifische Wärme (Luft, 300K) & $c_{p}$ & 1007 & $\mathrm{~J} \mathrm{~kg}^{-1} \mathrm{~K}^{-1}$ & {$[34]$} \\
spezifische Wärme (Wasser, $\left.20^{\circ} \mathrm{C}\right)$ & $c_{p}$ & 4182 & $\mathrm{~J} \mathrm{~kg}^{-1} \mathrm{~K}^{-1}$ & {$[34]$} \\
Wärmeleitfähigkeit (Luft, $300 \mathrm{~K})$ & $k$ & $26.2 \cdot 10^{-3}$ & $\mathrm{~W} \mathrm{~m}^{-1} \mathrm{~K}^{-1}$ & {$[34]$} \\
Wärmeleitfähigkeit (Wasser, $\left.20^{\circ} \mathrm{C}\right)$ & $k$ & 0.5984 & $\mathrm{~W} \mathrm{~m}^{-1} \mathrm{~K}^{-1}$ & {$[34]$} \\
Tait Parameter & $n_{\mathrm{T}}$ & 7.15 & - & {$[18]$} \\
statischer Druck & $p_{\text {stat }}$ & $1 \cdot 10^{5}$ & $\mathrm{~Pa}^{5}$ & \\
\hline
\end{tabular}

Tabelle C.2: Materialeigenschaften und Parameter. 



\section{Literaturverzeichnis}

[1] J. R. Blake und D. C. Gibson, 'Cavitation Bubbles Near Boundaries', Annu. Rev. Fluid Mech. 19, 99 (1987).

[2] A. Philipp und W. Lauterborn, 'Cavitation erosion by single laser-produced bubbles', J. Fluid Mech. 361, 75 (1998).

[3] A. Vogel, W. Lauterborn und R. Timm, 'Optical and acoustic investigations of the dynamics of laser-produced cavitation bubbles near a solid boundary', J. Fluid Mech. 206, 299 (1989).

[4] A. Vogel, J. Noack, G. Hüttman und G. Paltauf, 'Mechanisms of femtosecond laser nanosurgery of cells and tissues', Appl. Phys. B 81, 1015 (2005).

[5] M. J. K. Blomley, J. C. Cooke, E. C. Unger, M. J. Monaghan und D. O. Cosgrove, 'Microbubble contrast agents: a new era in ultrasound', BMJ 322, 1222 (2001).

[6] I. Lentacker, I. De Cock, R. Deckers, S.C. De Smedt und C. T. W. Moonen, 'Understanding ultrasound induced sonoporation: Definitions and underlying mechanisms', Adv. Drug Deliv. Rev. (2013), URL http://dx.doi.org/10.1016/j . addr. 2013.11.008.

[7] W. Lauterborn, 'Kavitation durch Laserlicht', Acustica 31, 51 (1974).

[8] S. Fujikawa und T. Akamatsu, 'Effects of the non-equilibrium condensation of vapour on the pressure wave produced by the collapse of a bubble in a liquid', J. Fluid Mech. 97, 481 (1980).

[9] U. Parlitz, V. Englisch, C. Scheffczyk und W. Lauterborn, 'Bifurcation structure of bubble oscillators', J. Acoust. Soc. Am. 88, 1061 (1990).

[10] W. Lauterborn und T. Kurz, 'Physics of bubble oscillations', Rep. Prog. Phys. 73, 106501 (2010). 
[11] Lord Rayleigh, 'On the Pressure Developed in a Liquid during the Collapse of a Spherical Cavity', Phil. Mag., Ser. 6 34, 94 (1917).

[12] T. G. Leighton, The Acoustic Bubble (Academic Press, London, 1994).

[13] F. R. Gilmore, 'The Growth or Collapse of a Spherical Bubble in a Viscous Compressible Liquid', Tech. Rep. Report No. 26-4, California Institute of Technology, Pasadena, California (1952).

[14] J. B. Keller und M. Miksis, 'Bubble oscillations of large amplitude', J. Acoust. Soc. Am. 68, 628 (1980).

[15] A. Prosperetti und A. Lezzi, 'Bubble dynamics in a compressible liquid. Part 1. First-order theory', J. Fluid Mech. 168, 457 (1986).

[16] A. Lezzi und A. Prosperetti, 'Bubble dynamics in a compressible liquid. Part 2. Second-order theory', J. Fluid Mech. 185, 289 (1987).

[17] D. Fuster, C. Dopazo und G. Hauke, 'Liquid compressibility effects during the collapse of a single cavitating bubble', J. Acoust. Soc. Am. 129, 122 (2011).

[18] M. Koch, C. Lechner, F. Reuter, K. Köhler, R. Mettin und W. Lauterborn, 'Numerical modeling of laser generated cavitation bubbles with the finite volume and volume of fluid method, using OpenFOAM', Computers \& Fluids 126, 71 (2016).

[19] C. Devin, 'Survey of Thermal, Radiation, and Viscous Damping of Pulsating Air Bubbles in Water', J. Acoust. Soc. Am. 31, 1654 (1959).

[20] I. Eick, Experimentelle und numerische Untersuchungen zur Dynamik sphärischer und asphärischer Kavitationsblasen, Dissertation, Technische Hochschule Darmstadt (1992).

[21] A. Gülhan und A. E. Beylich, 'Untersuchungen zur Dynamik von Gasblasen', Acustica 63, 276 (1987).

[22] R. I. Nigmatulin und N.S. Khabeev, 'Heat exchange between a gas bubble and a liquid', Fluid Dyn. 9, 759 (1974).

[23] A. Prosperetti, L. A. Crum und K. W. Commander, 'Nonlinear bubble dynamics', J. Acoust. Soc. Am. 83, 502 (1988). 
[24] R. Toegel, B. Gompf, R. Pecha und D. Lohse, 'Does Water Vapor Prevent Upscaling Sonoluminescence?', Phys. Rev. Lett. 85, 3165 (2000).

[25] J. O. Hirschfelder, C. F. Curtiss und R. B. Bird, Molecular Theory of Gases and Liquids (Wiley, New York, 1954).

[26] R. B. Bird, W. E. Stewart und E. N. Lightfoot, Transport Phenomena (Wiley, New York, 2002).

[27] W. Wagner und A. Pruß, 'The IAPWS Formulation 1995 for the Thermodynamic Properties of Ordinary Water Substance for General and Scientific Use', J. Phys. Chem. Ref. Data 31, 387 (2002).

[28] T. Fließbach, Statistische Physik (Spektrum, Heidelberg, 2010).

[29] J.A. Fay, Molecular Thermodynamics (Addison-Wesley, Reading, Massachusetts, 1965).

[30] W. Dreyer, F. Duderstadt, M. Hantke und G. Warnecke, 'Bubbles in liquids with phase transition. Part 1', Continuum Mech. Thermodyn. 24, 461 (2012).

[31] B. Han, K. Köhler, K. Jungnickel, R. Mettin, W. Lauterborn und A. Vogel, 'Dynamics of laser-induced bubble pairs', J. Fluid Mech. 771, 706 (2015).

[32] D. Schanz, B. Metten, T. Kurz und W. Lauterborn, 'Molecular dynamics simulations of cavitation bubble collapse and sonoluminescence', New J. Phys. 14, 113019 (2012).

[33] F. Franks, ed., Water - A comprehensive treatise, Bd. 1 (Plenum Press, New York, London, 1972).

[34] D. R. Lide, ed., CRC Handbook of Chemistry and Physics (CRC Press, 2004).

[35] N. Vargaftik, B. Volkov und L. Voljak, 'International Tables of the Surface Tension of Water', J. Phys. Chem. Ref. Data 12, 817 (1983).

[36] W. H. Press, S. A. Teukolsky, W. T. Vetterling und B.P. Flannery, Numerical Recipes in C (Cambridge University Press, 2002).

[37] W. Lauterborn und A. Vogel, Bubble Dynamics and Shock Waves (Springer, 2013), 
Bd. 8 von SHOCKWAVES, Kap. Shock Wave Emission by Laser Generated Bubbles, S. 67-103.

[38] A. Vogel, K. Nahen, D. Theisen und J. Noack, 'Plasma Formation in Water by Picosecond and Nanosecond Nd:YAG Laser Pulses-Part I: Optical Breakdown at Threshold and Superthreshold Irradiance', IEEE J. Quantum Electron. 2, 847 (1996).

[39] R. Geisler, Untersuchungen zur laserinduzierten Kavitation mit Nanosekunden- und Femtosekundenlasern, Dissertation, Universität Göttingen (2003).

[40] T. Kurz, D. Kröninger, R. Geisler und W. Lauterborn, 'Optic cavitation in an ultrasonic field', Phys. Rev. E 74, 066307 (2006).

[41] D. Kröninger, K. Köhler, T. Kurz und W. Lauterborn, 'Particle tracking velocimetry of the flow field around a collapsing cavitation bubble', Exp. Fluids 48 (2010).

[42] F. Hegedús, S. Koch, W. Garen, Z. Pandula, G. Paál, L. Kullmann und U. Teubner, 'The effect of high viscosity on compressible and incompressible Rayleigh-Plessettype bubble models', International Journal of Heat and Fluid Flow 42, 200 (2013).

[43] D. Obreschkow, M. Tinguely, N. Dorsaz, P. Kobel, A. de Bosset und M. Farhat, 'The quest for the most spherical bubble: experimental setup and data overview', Exp. Fluids 54, 1503 (2013).

[44] I. Akhatov, O. Lindau, A. Topolnikov, R. Mettin, N. Vakhitova und W. Lauterborn, 'Collapse and rebound of a laser-induced cavitation bubble', Phys. Fluids 13, 2805 (2001).

[45] K. Köhler, Numerische Untersuchungen zum optischen Durchbruch von FemtosekundenLaserpulsen in Wasser, Dissertation, Universität Göttingen (2010).

[46] WinLens, URL http://www.winlens.de.

[47] D. F. Gaitan, L. A. Crum, C. C. Church und R. A. Roy, 'Sonoluminescence and bubble dynamics for a single, stable, cavitation bubble', J. Acoust. Soc. Am. 91, 3166 (1992).

[48] L. A. Crum, 'Resource Paper: Sonoluminescence', J. Acoust. Soc. Am. 138, 2181 (2015). 
[49] M. P. Brenner, S. Hilgenfeldt und D. Lohse, 'Single-bubble sonoluminescence', Rev. Mod. Phys. 74, 425 (2002).

[50] P. Koch, T. Kurz, U. Parlitz und W. Lauterborn, 'Bubble dynamics in a standing sound field: The bubble habitat', J. Acoust. Soc. Am. 130, 3370 (2011).

[51] K. S. Suslick, 'Sonochemistry', Science 247, 1439 (1990).

[52] T. Lepoint, F. Lepoint-Mullie und A. Henglein, Sonochemistry and Sonoluminescence (Kluwer Academic, Dordrecht, 1999), Kap. Single Bubble Sonochemistry, S. 285290.

[53] T. Verraes, F. Lepoint-Mullie, T. Lepoint und M. S. Longuet-Higgins, 'Experimental study of the liquid flow near a single sonoluminescent bubble', J. Acoust. Soc. Am. 108, 117 (2000).

[54] G. E. Vazquez und S. J. Putterman, ‘Temperature and Pressure Dependence of Sonoluminescence', Phys. Rev. Lett. 85, 3037 (2000).

[55] K. Ebeling, 'Zum Verhalten kugelförmiger, lasererzeugter Kavitationsblasen in Wasser', Acustica 40, 229 (1978).

[56] W. Hentschel und W. Lauterborn, 'Acoustic emission of single laser-produced cavitation bubbles and their dynamics', Applied Scientific Research 38, 225 (1982).

[57] S. Koch, W. Garen, F. Hegedüs, W. Neu, R. Reuter und U. Teubner, 'Time-resolved measurements of shock-induced cavitation bubbles in liquids', Appl. Phys. B 108, 345 (2012).

[58] B. D. Storey und A. J. Szeri, in Proceedings of the Royal Society of London A: Mathematical, Physical and Engineering Sciences (The Royal Society, 2000), Bd. 456, S. 1685-1709.

[59] L. Stricker, A. Prosperetti und D. Lohse, 'Validation of an approximate model for the thermal behavior in acoustically driven bubbles', The Journal of the Acoustical Society of America 130, 3243 (2011).

[60] E. Jones, T. Oliphant, P. Peterson et al., SciPy: Open source scientific tools for Python (2001-), [Online; accessed 2015-07-21], URL http://www.scipy.org/

[61] O. Lindau, Untersuchungen zur lasererzeugten Kavitation, Dissertation, Universität Göttingen (2001). 
[62] A. Vogel, J. Noack, K. Nahen, D. Theisen, S. Busch, U. Parlitz, D. X. Hammer, G. D. Noojin, B. A. Rockwell und R. Birngruber, 'Energy balance of optical breakdown in water at nanosecond to femtosecond time scales', Appl. Phys. B 68, 271 (1999).

[63] A. Vogel und W. Lauterborn, 'Ein Raumfrequenzfilterungsverfahren zur Untersuchung der Strömungsverhältnisse in der Umgebung von Kavitationsblasen', Optik 69, 176 (1985).

[64] M. Dular und O. Coutier-Delgosha, 'Thermodynamic effects during growth and collapse of a single cavitation bubble', J. Fluid Mech. 736, 44 (2013).

[65] P. A. Quinto-Su, M. Suzuki und C. D. Ohl, 'Fast temperature measurement following single laser-induced cavitation inside a microfluidic gap', Scientific Reports 4, 5445 (2014).

[66] V. K. Natrajan und K. T. Christensen, 'Two-color laser-induced fluorescent thermometry for microfluidic systems', Meas. Sci. Technol. 20, 015401 (2009).

[67] J. Crimaldi, 'Planar laser induced fluorescence in aqueous flows', Exp. Fluids 44, 851 (2008).

[68] J. Sakakibara, K. Hishida und M. Maeda, 'Measurements of thermally stratified pipe flow using image-processing techniques', Exp. Fluids 16, 82 (1993).

[69] P. W. Milonni und J. H. Eberly, Lasers (Wiley, New York, 1988).

[70] D. Dabiri, 'Digital particle image thermometry/velocimetry: a review', Exp. Fluids 46, 191 (2009).

[71] D. J. Flannigan und K. S. Suslick, 'Plasma formation and temperature measurement during single-bubble cavitation', Nature 434 (2005).

[72] E. B. Flint und K.S. Suslick, 'The Temperature of Cavitation', Science 253, 1397 (1991).

[73] W. B. McNamara, Y. T. Didenko und K. S. Suslick, 'Sonoluminescence temperatures during multi-bubble cavitation', Nature 401, 772 (1999).

[74] G.S. Settles, Schlieren and shadowgraph techniques: visualizing phenomena in transparent media (Springer, Berlin, Heidelberg, New York, 2001). 
[75] A. Zijlstra und C. D. Ohl, ‘On fiber optic probe hydrophone measurements in a cavitating liquid (L)', J. Acoust. Soc. Am. 123, 29 (2008).

[76] S. Müller, P. Helluy und J. Ballmann, 'Numerical simulation of a single bubble by compressible two-phase fluids', Int. J. Numer. Meth. Fluids 62, 591 (2010).

[77] M. Bachmann, Dynamics of Cavitation Bubbles in Compressible Two-Phase Fluid Flow, Dissertation, RWTH Aachen (2012).

[78] O. Baghdassarian, B. Tabbert und G. A. Williams, 'Luminescence from laser-created bubbles in cryogenic liquids', Phys. Rev. E 75, 066305 (2007).

[79] H. Söhnholz, Akustische Blasenfallen und laserinduzierte Kavitation, Diplomarbeit, Universität Göttingen (2010).

[80] H. Kuttruff, Akustik (Hirzel, Stuttgart, 2004). 



\section{Danksagung}

Viele Leute haben zum Gelingen dieser Arbeit beigetragen. Ich möchte mich besonders bedanken bei ...

- Prof. Dr. Christoph F. Schmidt als Betreuer und Referent,

- Prof. Dr. Ulrich Parlitz für die Übernahme des Korreferats,

- Dr. Thomas Kurz für die Betreuung und viele hilfreiche Diskussionen,

- Prof. Dr. Werner Lauterborn für die Motivation, hilfreiche Kommentare, zahlreiche anregende Diskussionen und das Korrekturlesen der Arbeit,

- Dr. Robert Mettin für viele hilfreiche Diskussionen,

- der Feinmechanischen Werkstatt unter der Leitung von Dieter Hille sowie der Elektronik-Werkstatt unter Dr. Karl Lautscham für die unkomplizierte Hilfe bei experimentellen Aufbauten,

- Thomas Geiling und Hans Werner Strube für die Unterstützung bei allen IT-Fragen,

- allen Mitgliedern der Arbeitsgruppe Ultraschall, Kavitation und Nichtlineare Systeme (auch den ehemaligen) am Dritten Physikalischen Institut,

- Jutta Gerber-Nolte aus der Fakultät für Chemie für die Bereitstellung des Spektrometers,

- der Deutschen Forschungsgemeinschaft für finanzielle Unterstützung,

- Dr. Georg Herink aus dem IV. Physikalischen Institut für das Korrekturlesen und

- bei meiner Familie, die mich jederzeit unterstützt hat. 



\section{Lebenslauf}

Persönliche Daten:

Name: Hendrik Söhnholz

Geburtsdatum: 28.07.1982

Geburtsort: Großburgwedel

Nationalität: deutsch

Akademischer und beruflicher Werdegang:

seit 09/2016 Systemanalytiker (HPC) bei der GNS Systems GmbH, Braunschweig

06/2010 - 08/2016 wissenschaftlicher Mitarbeiter im 3. Physikalischen Institut, Georg-August-Universität Göttingen

05/2009-11/2009 Java-Softwareentwickler und Linux-Serveradministrator bei intranda Software, Göttingen

01/2006 - 08/2016 studentische Hilfskraft im CIP Pool der Fakultät für Physik, Georg-August-Universität Göttingen

10/2003 - 05/2010 Physikstudium an der Georg-August-Universität Göttingen mit Nebenfach Informatik

07/2002-03/2003 Grundwehrdienst

2002 Abitur am Gymnasium Walsrode 